\title{
Optical phonons in colloidal CdSe nanorods
}

\author{
vorgelegt von \\ Diplom-Physiker \\ Holger Lange \\ aus Bonn-Bad Godesberg
Von der Fakultät II - Mathematik und Naturwissenschaften der Technischen Universität Berlin
zur Erlangung des akademischen Grades
Doktor der Naturwissenschaften
Dr.rer.nat.

genehmigte Dissertation

Promotionsausschuss:

Vorsitzender: Prof. Dr. Michael Lehmann

Berichter: Prof. Dr. Christian Thomsen

Berichter: Prof. Dr. Ulrike Woggon

Tag der wissenschaftlichen Aussprache: 5.11.2009

Berlin 2009

D 83 

"Optical phonons in colloidal CdSe nanorods"

Holger Lange, 2009

Hiermit versichere ich an Eides statt, dass ich die vorliegende Dissertation selbständig verfasst habe. Alle benutzen Quellen sind in der Dissertation aufgeführt. Teile der Disertation wurden schon veröffentlicht.

Berlin, den 13. Oktober 2009

"Optical phonons in colloidal CdSe nanorods"

Holger Lange, 2009

Die Anmeldung der Promotionsabsicht ist weder früher noch gegenwärtig bei einer anderen Hochschule erfolgt.

Berlin, den 13. Oktober 2009 



\section{List of Publications}

Parts of this thesis have already been published:

Uniaxial strain in $\mathrm{CdSe}-\mathrm{ZnS}$ core-shell nanorods.

$\mathrm{H}$. Lange, M. Mohr, M. Artemyev, and C. Thomsen.

In preparation.

Temperature dependent phonon processes in colloidal CdSe nanocrystals.

P. Kusch, H. Lange, M. Artemyev, and C. Thomsen.

Submitted to Phys. Rev. B.

Raman investigation of strain effects in CdSe nanorods.

H. Lange, M. Artemyev, U.Woggon, and C. Thomsen.

In print (phys. stat. sol. b).

Thin-walled $\mathrm{Er}^{3+}: \mathrm{Y}_{2} \mathrm{O}_{3}$ nanotubes showing up-converted fluorescence.

C. Erk, S.M. Caba, H. Lange, S. Werner, C. Thomsen, M. Steinhart, A. Berger, and S. Schlecht.

Phys. Chem. Chem. Phys. 11, 3623 (2009).

Geometry dependence of the phonon modes in CdSe nanorods.

$\mathrm{H}$. Lange, M. Artemyev, U. Woggon, and C. Thomsen.

Nanotechnology 20, 045705 (2009).

Direct Observation of the Radial Breathing Mode in CdSe Nanorods.

$\mathrm{H}$. Lange, M. Mohr, M. Artemyev, U. Woggon, and C. Thomsen.

Nano Letters 8, 4614 (2008).

Experimental investigation of exciton-LO-phonon couplings in $\mathrm{CdSe} / \mathrm{ZnS}$ core/shell nanorods.

H. Lange, M. Artemyev, U. Woggon, T. Niermann, and C. Thomsen.

Phys. Rev. B 77, 193303 (2008).

Effect of $\mathrm{ZnS}$ shell on the Raman spectra from CdSe nanorods.

$\mathrm{H}$. Lange, M. Machon, M. Artemyev, U. Woggon, and C. Thomsen.

phys. stat. sol (RRL) 1, 274 (2007). 



\begin{abstract}
Colloidal CdSe nanorods are nanoparticles synthesized in solution. The synthesis allows the growth of CdSe nanorods with well defined diameters and aspect ratios. They have many potential applications in the field of optoelectronics and biotechnology. The nanorods can be epitaxially covered with a graded $\mathrm{CdS} / \mathrm{ZnS}$ shell of a few monolayers in thickness. The shell leads to an increased quantum efficiency and improved photostability of the nanorods. However, the lattice mismatch between the nanorod core material and the shell material introduces strain into the core lattice.

In this work Raman spectroscopy, accompanied by ab-initio calculations, is used to determine the amount of this strain, the exciton-phonon coupling strength in the nanorods and to investigate confinement effects.

The longitudinal optical phonons in a nanorod are confined to the nanorod volume. The confinement of a phonon wavefunction leads to a neutralization of the $\mathbf{q}=0$ rule and the phonon frequency is found to depend on the nanorod diameter. The coupling strength between longitudinal optical phonons and excitons is investigated. It also depends on the nanorod diameter. The total coupling strength is much lower than in bulk material due to a decrease of the influence of the Coulomb interaction in nanoparticles. However, the coupling strength is found to rise for decreasing diameters. This is due to the increasing contributions of higher frequency phonons for smaller nanoparticle sizes.

A radial breathing mode with a diameter dependent frequency is deduced from $a b$-initio calculations and its existence in nanorods is verified experimentally. The diameter-dependence of the modes' frequency can be used to estimate the nanorod diameter from a Raman measurement.

In core-shell structures, the coverage of a CdSe nanorod with a $\mathrm{ZnS}$ shell leads to a compressive strain of the CdSe core due to the smaller lattice parameter of ZnS compared to CdSe. Ab-initio calculations show that all bonds of the CdSe core are shortened. The bonds in the lateral direction are much more strongly compressed than the bonds parallel to the c-axis. The amount of strain is estimated from the Raman spectra. The compressive nature of the shell decreases for thicker nanorods. The exciton wave function changes with the modified boundary from air to ZnS. This is reflected in a altered exciton-phonon coupling strength and can be monitored in the Raman spectra.
\end{abstract}




\section{Zusammenfassung}

CdSe Nanorods sind Nanopartikel, die in einer kolloidalen Lösung synthetisiert werden. Für diese existieren viele mögliche Anwendungen im Bereich der Optoelektronik und der Biotechnologie. Der Durchmesser und die Länge der Nanorods lässt sich durch die Syntheseparameter festlegen. Die Nanorods können in eine epitaktische Hülle aus einem Halbleitermaterial mit einer größeren Bandlücke, ZnS, eingebettet werden. Diese Hülle verbessert die optischen Eigenschaften und ermöglicht eine weitere Funktionalisierung der Oberfläche. Der Unterschied der Gitterparameter zwischen ZnS und CdSe führt jedoch zu Verspannungen im CdSe Kern.

In dieser Arbeit werden der Einfluss der ZnS Hülle auf den CdSe Kern und die Schwingungseigenschaften von CdSe Nanorods mit Ramanspektroskopie und $a b$ initio Berechnungen untersucht. Mit Ramanspektroskopie wird gezeigt, dass die Frequenzen der longitudinal-optischen Phononen vom Durchmesser der Nanorods bestimmt werden. Dies liegt daran, dass die Wellenfunktion der Phononen im Nanokristall eingeschlossen ist. Dadurch wird die $\mathbf{q}=0$-Regel aufgehoben. Die Kopplung der longitudinal-optischen Phononen an Exzitonen hängt ebenfalls vom Durchmesser ab. Die Kopplungsstärke in Nanorods ist viel kleiner als im Volumenmaterial, nimmt jedoch für abnehmende Durchmesser zu. Dies hängt mit einem stark verminderten Einfluss der Coulomb-Wechselwirkung gegenüber Volumenmaterial und einer steigenden Kopplung an hochfrequente Phononen für abnehmende Durchmesser zusammen.

Als dritten durchmesserabhängigen Effekt gibt es eine radiale "Atmungsmode" in den Nanorods. Die Frequenz dieser Schwingungsmode hängt stark vom NanorodDurchmesser ab, und die Messung der Frequenz erlaubt die Bestimmung des Durchmessers mittels Ramanspektroskopie.

Die durch die Hülle verursachten Verpannungen werden mit ab-initio-Berechnungen untersucht. Bindungen entlang der c-Achse sind kaum verspannt, die lateralen Bindungen sind homogen verspannt. Die Größe der Verspannungen kann quantitativ mit Ramanspektroskopie bestimmt werden. Der Einflus der Hülle schwindet für größere Nanorod-Durchmesser. Das durch die Hülle geänderte Nanorod-Umfeld, ZnS statt Luft, führt zu einer Änderung der Exziton-Wellenfunktion und dadurch zu einer stark abnehmenden Exziton-Phonon-Kopplung. 


\section{Contents}

1 Introduction $\quad 1$

2 CdSe nanorods $\quad 5$

2.1 Cadmium selenide . . . . . . . . . . . . . . . . 5

2.2 Colloidal nanorod growth . . . . . . . . . . . . . . . 6

2.2.1 TEM analysis . . . . . . . . . . . . . . . 10

2.3 Core-shell nanorods . . . . . . . . . . . . . . . . . . . . . . 13

2.3.1 Luminescence properties . . . . . . . . . . . . 15

3 Methods $\quad 19$

3.1 Phonons in crystalline semiconductors . . . . . . . . . . . 20

3.2 Density functional theory, ab-initio calculations . . . . . . . . . 22

3.2 .1 SIESTA . . . . . . . . . . . . . . . 24

3.2.2 Ab-initio calculations of bulk CdSe and CdSe nanorods . . . . 25

3.3 Raman spectroscopy . . . . . . . . . . . . . . . . . . . 30

3.3.1 Raman spectroscopy of CdSe nanorods . . . . . . . . . . 32

4 Geometry dependence of the phonon modes in CdSe nanorods $\quad 37$

4.1 Size-dependent Raman spectra . . . . . . . . . . . . . . . . . . . . . 37

4.2 Phonon confinement . . . . . . . . . . . . . . . . . 41

4.3 Surface optical phonons . . . . . . . . . . . . . . . . . 46

5 Exciton-LO-phonon coupling in CdSe nanorods 51

5.1 The Huang-Rhys parameter . . . . . . . . . . . . . . . . . . 51

5.2 Exciton-phonon coupling in Raman spectra . . . . . . . . . . . . . 54

5.3 Huang-Rhys parameter of CdSe nanorods . . . . . . . . . . . . . 57

6 The Radial breathing mode in CdSe nanorods $\quad 65$

6.1 Motivation . . . . . . . . . . . . . . . 65

6.2 Linear elasticity theory . . . . . . . . . . . . . . . . . 67

$6.3 \quad$ Ab-initio calculations . . . . . . . . . . . . . . . . . 68 
6.4 Raman measurement of the radial breathing mode . . . . . . . . . . . 69

7 Core-shell nanorods $\quad \mathbf{7 5}$

7.1 Influence of the shell on the Raman spectra . . . . . . . . . . . . 75

7.2 Strain in core-shell nanorods . . . . . . . . . . . . . . . 76

7.3 Surface optical phonons in core-shell nanorods . . . . . . . . . . . . . 82

7.4 Shell-influence on the exciton-phonon coupling . . . . . . . . . . . . 83

7.5 The RBM in core-shell nanorods . . . . . . . . . . . . . . . . . . . . . 84

$\begin{array}{llr}8 & \text { Summary and outlook } & 87\end{array}$

$\begin{array}{ll}\text { Bibliography } & 91\end{array}$ 


\section{Chapter 1}

\section{Introduction}

Colloidal nanocrystals are inorganic structures from particles of a few hundred atoms up to almost $\mu \mathrm{m}$ sized structures, which are synthesized in solution. They can be grown from many different materials and can be cheaply produced in fairly large amounts and with a high homogeneity in size and shape[1]. The possibility to tune the dimensional regime of the nanocrystals is an important achievement in materials science, since at the nanoscale both the size and the shape dictate the chemical and physical (such as catalytic and optical) properties of the material[24]. Because of their intrinsic robustness and their potential ease of integration with existing technologies, colloidal nanocrystals have been intensively developed and investigated during the last years. Besides potential technological applications, they are a versatile model system for fundamental research, e.g. to investigate size- and shape-dependent confinement effects. Spheres[5], cubes[6], rods[7], disks[8], wires[9] and other non-trivial shapes like tetrapods[10] or arrows[11] can be prepared from a wide variety of materials.

Of these, nanorods of the direct band-gap II-VI semiconductor CdSe are especially interesting. The correct choice of the growth parameters results in CdSe nanorods with well defined diameters and aspect ratios, while the amount of nanorods created is highly scalable[12]. CdSe nanorods with defined diameters down to $2 \mathrm{~nm}$ and aspect ratios up to 30:1 can be achieved in large scales and with narrow size distributions[13]. They have a size-dependent photoluminescence that covers the whole visible spectrum $[14,15]$.

Their unique optical and electronic properties offer a huge potential for applications in the field of optoelectronics. For example, hybrid solar cells can be constructed from CdSe nanorods embedded in polymers[16]. In a prototype, the control of the nanorod length allows to change the distance on which electrons are transported through the device. Tuning the diameter of the nanorod and the associated band 
Chapter 1. Introduction

gap then allows the optimization of the absorption spectrum to the solar emission spectrum. Another suggested application is to electronically contact and couple quantum dot based photonic devices by using CdSe nanorods. Quantum dots and nanorods can be connected via tunable potential barriers and arranged in threedimensional space[17]. CdSe nanorods can also form a liquid crystalline phase with an orientational order. They have much higher elastic constants than the common organic liquid crystal molecules. The orientation of these liquid crystals is very sensitive to an external field[18]. Because CdSe nanorods show a linearly polarized photoluminescence[19] the nanorods in liquid phase have potential applications as functional components in electrooptical devices such as displays and electrooptic modulators.

The nanorods can be coated with an epitaxially, graded CdS/ZnS shell of one to six monolayers in thickness[11]. The coated nanorods have an increased quantum efficiency, are more stable in air and under visible or UV excitation[20] and they can be optically excited for a polarized stimulated emission[21]. The ZnS shell enables further surface functionalization, allows water solubility and the embedment in biological systems[22]. This gives raise to applications in the field of biotechnology. For example, CdSe-ZnS core-shell nanocrystals in phospholipid block-copolymer micelles can be used for both, in vitro and in vivo imaging. When conjugated to DNA, these nanocrystal-micelles act as fluorescent probes to hybridize to specific complementary sequences. When e.g. injected into Xenopus embryos, the nanocrystal-micelles are stable, nontoxic, and cell autonomous. The nanocrystals' fluorescence can be followed, allowing lineage-tracing experiments in embryogenesis[23]. Bioconjugated core-shell nanoparticles are also suitable for targeting and imaging of human cancer cells in living tissue. This class of nanoparticle conjugates contains targeting-ligands for tumor antigen recognition. Both passive tumor targeting (due to enhanced permeation and retention of the nanoparticles at tumor sites) and active tumor targeting (due to rapid nanoparticle-antibody binding to tumor-specific antigens) can be achieved. This opens new possibilities for sensitive and simultaneous imaging of multiple biomarkers involved in cancer metastasis and invasion[24].

However, many properties of these new structures are still unknown. The small sizes of the nanorods make a systematic investigation difficult. The sizes of the nanocrystals requires specialized techniques for a characterization.

In this work Raman spectroscopy, accompanied by ab-initio calculations, is applied to characterize the vibrational properties of the nanorods and to investigate sizedependent effects. Longitudinal optical phonons in the nanorods are found to be confined and the dependencies are investigated. The coupling strength between longitudinal optical phonons and excitons does depend on the nanorod geometry. In core-shell structures, the signal stemming from the shell can be well separated from the core related Raman signal. Changes in the nanorod core due to the addition of the shell can be monitored by observing the changes in the core related Raman 
signal. The ZnS shell leads to an uniaxial compression of the CdSe core, the amount of strain is estimated from the Raman spectra. The exciton wave function changes with the changed boundary. This is reflected in a changed exciton-phonon coupling strength and can also be monitored in the Raman spectra. 



\section{Chapter 2}

\section{CdSe nanorods}

\subsection{Cadmium selenide}

Cadmium selenide (CdSe) is a solid binary compound of cadmium (Cd) and selenium (Se). CdSe can be present in three cristaline phases: hexagonal wurtzite, cubic zincblende and cubic rock-salt. The phase of CdSe that is stable at ambient conditions belongs to the wurtzite type and occurs in form of the mineral cadmoselite[25] (see Figure 2.1). Zincblende-type CdSe is metastable at ambient conditions. Mechanical grinding of wurtzite-type CdSe can induce the formation of the zincblende-type arrangement[26]. Under increasing pressure, the wurtzite structure transforms to the rocksalt structure[27].

Wurtzite CdSe crystallizes in space group $P 6_{3} m c$ with the lattice parameters $a=$ $4.302 \AA$ and $c=7.014 \AA$. CdSe in the wurtzite crystal structure is a direct bandgap II-VI semiconductor. The exciton binding energy is $13.2 \mathrm{meV}$ and the energy of the excitonic transition is $1.82 \mathrm{eV}[28]$. II-VI semiconductors can usually be doped either $\mathrm{n}$ or $\mathrm{p}$ type, but not both. CdSe in particular is a good n-type conductor, while hard to dope p-type[29]. However, p-type doping with rather small hole concentrations $\left(\approx 10^{17} \mathrm{~cm}^{-3}\right.$ ) can, for example, be achieved using nitrogen as dopant in a molecular beam epitaxy growth set-up[30]. The problem of doping can be dealt with when integrating CdSe nanostructures in heterostructures with materials that can be doped oppositional e.g. silicon[31]. 


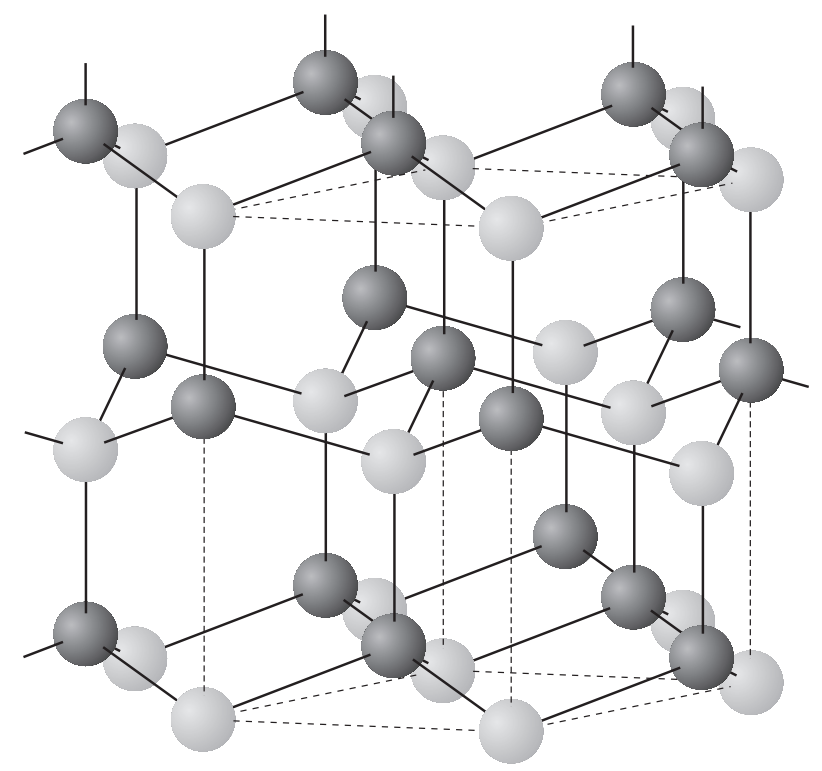

Figure 2.1: Wurtzite crystal structure. Cd and Se atoms both occupy Wyckoff position $2(b) 1 / 3,2 / 3, z$ with $z=0$ and $z=0.37596(4)$, respectively.

\subsection{Colloidal nanorod growth}

CdSe nanorods are synthesized via the most widely used approach for the synthesis of colloidal nanocrystals[2, 7, 13, 32]. A typical synthesis system consists of three components: precursors, organic surfactants and solvents. In a frequently used setup, the surfactant is in solution in a three-neck flask and heated in an Ar atmosphere. The temperature is measured with a thermometer in the solution and the solution is mixed with a stirrer. The precursors are then quickly injected with a syringe. Upon heating the reaction medium to a sufficiently high temperature, the precursors chemically transform into active atomic or molecular species (monomers); these then form nanocrystals whose subsequent growth is greatly affected by the presence of surfactant molecules.

The formation of the nanocrystals involves two steps: nucleation of an initial seed and growth. In the nucleation step, precursors decompose or react at a relatively high temperature to form a supersaturation of monomers followed by a nucleation of nanocrystals. These nuclei then grow by incorporating additional monomers still present in the reaction medium. This only generates nanoparticles that will be crystalline solids if the constituent atoms can rearrange and anneal during the growth. The temperature for the growth has to be hot enough to allow the rearrangement of the atoms and the annealing within a growing nanocrystal over the course of 
the synthesis. The precursors need to rapidly decompose or react at the required growth temperature to yield reactive atomic or molecular species (the monomers), which then cause nanocrystal nucleation and growth. The energy with which surfactant molecules present in the growth medium adhere to the surfaces of the growing nanocrystals needs to be such that it allows a dynamic solvation at the growth temperature. The surfactant needs to be able to exchange on and off the growing crystals, so that regions of the nanocrystal surface are transiently accessible for growth, yet entire crystals are, on average, monolayer-protected to block aggregation.

The surfactant molecules do not only bind to the growing nanocrystal surface, but also form a complex with the reactive monomer species. The stability and diffusion rate of these complexes, as well as the binding strength of the surfactant molecules to the growing nanocrystal surface, are all strongly temperature dependent. Increasing the temperature greatly decreases the stability of the intermediate complexes formed in solution and the binding of the surfactants to the nanocrystal surface, while increasing the diffusion rates of the complexes. This favors the nucleation and growth of the nanocrystals. However, too high temperatures lead to an uncontrolled growth so that it is impossible to exploit kinetic effects to achieve precise control over the size and the size distribution of the nanocrystals. Choosing an appropriate temperature range is one of the key steps in obtaining control over colloidal nanocrystal growth.

To produce nanocrystals with a relatively narrow size distribution the concept of size-distribution focusing is used, which is based on the fact that small crystals will grow more rapidly than larger ones, if the monomer concentrations are sufficiently high. Very small crystals are unstable owing to their large fraction of active surface atoms. Larger crystals with a smaller surface-to-volume ratio are stable and grow. There exists a critical size, where nanocrystals neither grow nor shrink. Such a critical size exists at any given monomer concentration. At a high monomer concentration, the critical size is small so that all the particles grow. In this situation, smaller particles grow faster than the larger ones, and as a result, the size distribution can be focused down to one that is nearly monodisperse. If the monomer concentration is below a critical threshold, small nanocrystals are depleted as larger ones grow and the size distribution broadens, or defocuses. Thus, when the monomer concentration is depleted owing to growth, the critical size becomes larger than the average size present, and the distribution broadens. This means that nucleation must occur on a short time scale. This is achieved by rapidly injecting suitable precursors into the solvent at high temperature to generate transient supersaturation in monomers and induce a nucleation burst. A rapid and intense nucleation burst will lower the monomer concentration below the nucleation threshold, so monomers remaining in solution will only add to the existing nuclei.

Compared with equilibrium nanocrystals with nearly round shapes, nanocrystals 
with highly anisotropic shapes, such as nanorods, have larger surface areas, which renders them metastable. The formation of these metastable nanocrystals thus requires a kinetic growth regime, whereas equilibrium nanocrystals with low aspect ratios are obtained in the slow growth limit under thermodynamic control. At low growth rate, nearly round nanocrystals are formed with a broad size distribution. At higher growth rate, focusing is observed. When the growth rate is increased just beyond the focusing regime, nanorods can be obtained. The equilibrium shape of inorganic nanocrystals, although faceted, has a low aspect ratio both because this minimizes the surface area and because the surface energy of the low-energy facets of the crystal are relatively close to each other in energy. However, the growth rate of a crystal facet depends exponentially on the surface energy, so that at high growth rates, in a kinetically controlled growth regime, high-energy facets grow more quickly than low-energy facets, as schematically displayed in Figure 2.2 (I). Such a facet may form transiently during the growth of the high-energy facet. Once such a facet forms, it will persist, replacing the initial high-energy facet. This new intermediate energy facet will still grow more quickly than the initial slow-growing one, so that the shape will evolve in a complex pattern during growth. The onset of kinetic shape control can be adjusted using selective adhesion (2.2 (II)). Organic surfactants exchange on the nanocrystal's surface during growth. In a faceted crystal, the exchange rate on the different facets need not be the same. The introduction of an organic molecule that selectively adheres to a particular crystal facet can be used to effectively lower the energy and slow the growth rate of that facet relative to others. For CdSe, for example, Puzder et al. calculated the binding energy of various organic ligands to the facets of an initial CdSe nanocrystal seed[33]. The surfactants bind more strongly to the facets along the c-axis than to the other facets. A proper choice of the ligands thus facilitates the nanorod growth and this growth then occurs on the Cd-dominated (0001h) facet.

When the desired shape and size has developped, the synthesis is stopped by lowering the reaction temperature. Then a surfactant coating layer around the nanorods remains tightly bound to their surface and provides their full solubility in a variety of solvents.

The synthesis of the nanorods investigated in this work is performed by Mikhail Artemyev $^{1}$. To grow the standard CdSe nanorods, dimethylcadmium is used as $\mathrm{Cd}$ precursor and trioctylphosphine selenide as Se precursor. Both are injected quickly in the reaction mixture. Trioctylphosphine oxide is the solvent, heated to $300{ }^{\circ} \mathrm{C}$ and hexadecylphosphonic acid is acting as surface growth modifier. The nanorods grow at high temperatures and the reaction is stopped by fast cooling.

The extra-thin nanorods for the strain-investigation in Chapter 7 are synthesized

\footnotetext{
${ }^{1}$ Present affiliation: Institute for Physico-Chemical Problems of Belorussian State University, Minsk, Belarus.
} 


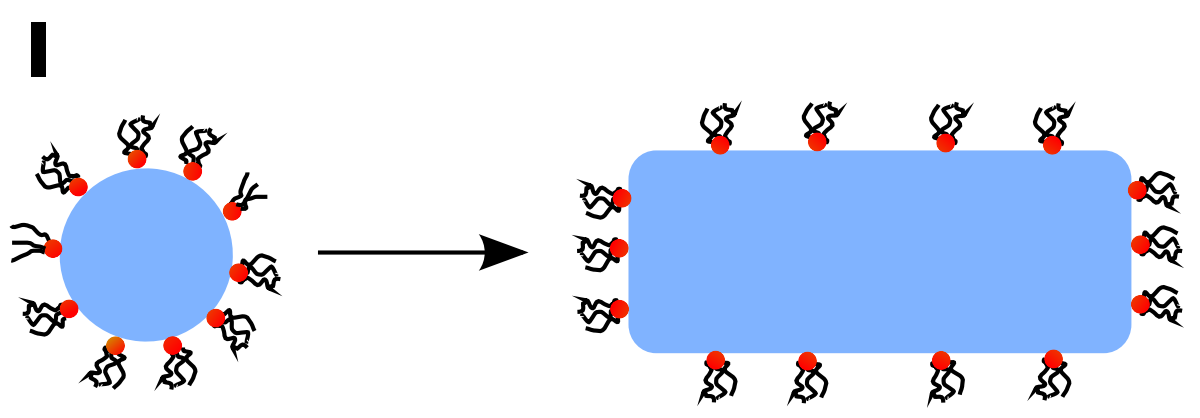

II
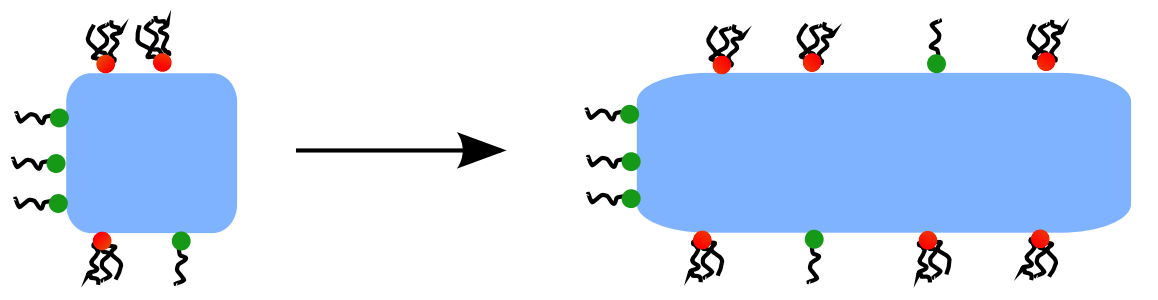

Figure 2.2: Kinetic shape control at high growth rate (I). The high-energy facets grow more quickly than low-energy facets in a kinetic regime. Kinetic shape control through selective adhesion (II). The introduction of an organic molecule that selectively adheres to a particular crystal facet can be used to slow the growth of that side relative to others, leading to the formation of rod-shaped nanocrystals.

from cadmium acetate that is mixed with dodecylphosphonic acid and trioctylphosphine oxide. Elemental Se is dissolved in trioctylphosphine in an inert atmosphere and the resultant solution is injected quickly to the reaction mixture at $260{ }^{\circ} \mathrm{C}$. The developing CdSe core nanorods are then allowed to grow at slightly lower temperatures than the standard nanorods, $240{ }^{\circ} \mathrm{C}$, and the reaction is stopped by fast cooling below $180{ }^{\circ} \mathrm{C}$. The sizes of these small nanorods are not characterized by electron microscopy, but by absorption and photoluminescence measurements. The mean diameter can be estimated by these methods. The exact diameter distribution, however, is not known.

All nanorod samples can be isolated from the reaction mixture and then redispersed in a variety of solvents.

The size and shape of the nanorods is mainly controlled by the relative amount of the base materials and the reaction time. To allow a systematic investigation of size-dependend effects, nanorods of different diameters with multiple aspect-ratios are grown. 
Chapter 2. CdSe nanorods

\subsubsection{TEM analysis}

The sizes, size distributions and crystalline structure of the nanorods can be investigated with transmission electron microscopy (TEM). All TEM micrographs are courtesy of Tore Niermann ${ }^{2}$. Figure 2.3 displays an exemplary TEM micrograph of a CdSe nanorod sample of nanorods with $6 \mathrm{~nm}$ diameter and $35 \mathrm{~nm}$ length. Like in this example, all investigated nanorod samples are homogeneous and feature a narrow size distribution.

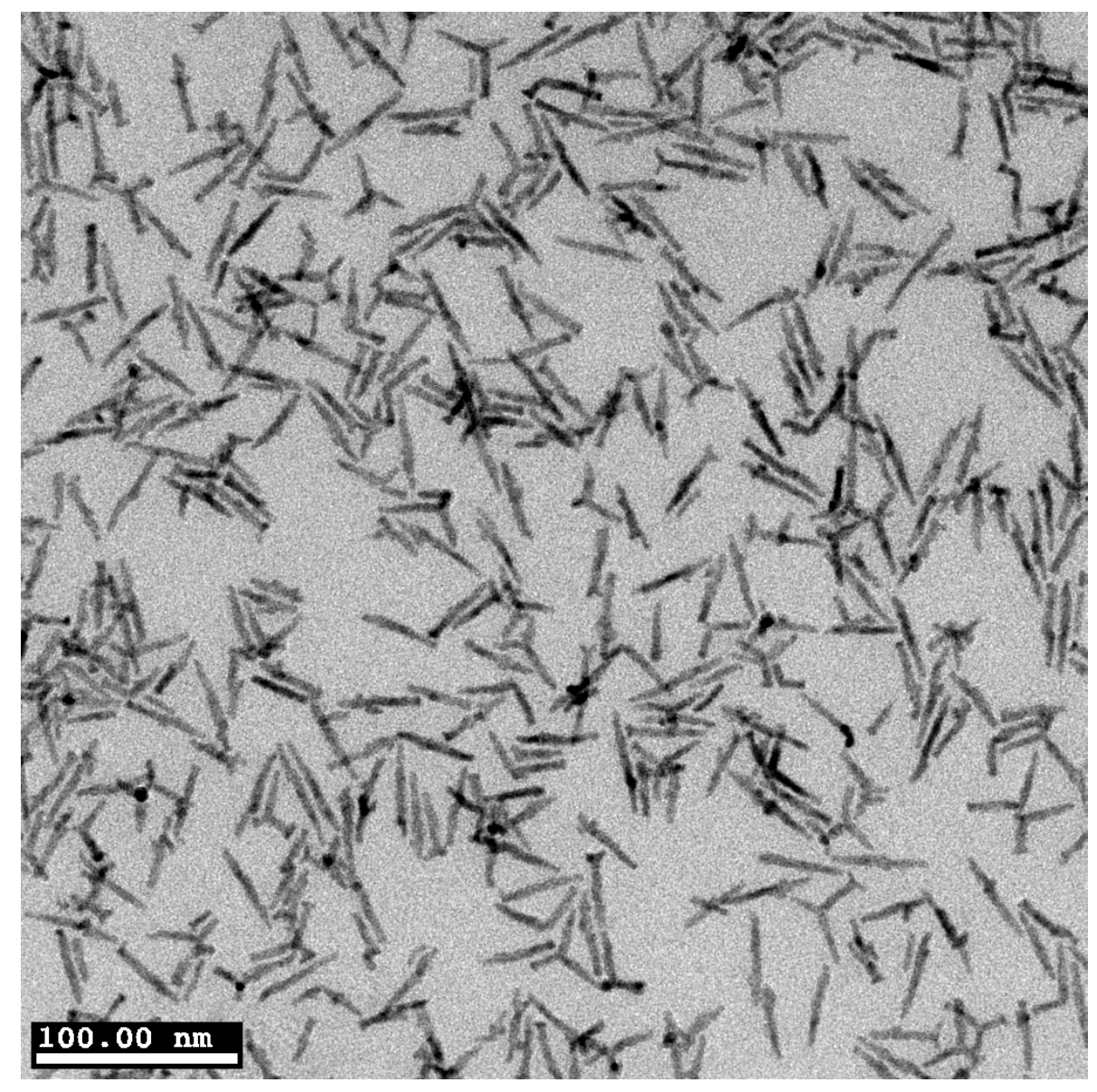

Figure 2.3: TEM micrograph of an exemplary CdSe nanorod ensemble.

No contaminations with diverse shaped crystals are present. The nanorods are of uniform size und randomly orientated. Depending on the density of the nanorod solution, the nanorods tend to form clusters.

\footnotetext{
${ }^{2}$ Present affiliation: Institut für Optik und Atomare Physik, Technische Universität Berlin.
} 
A high resolution transmission electron microscope (HR-TEM) micrograph of a cutout of the sample displayed in Figure 2.3 is shown in Figure 2.4.

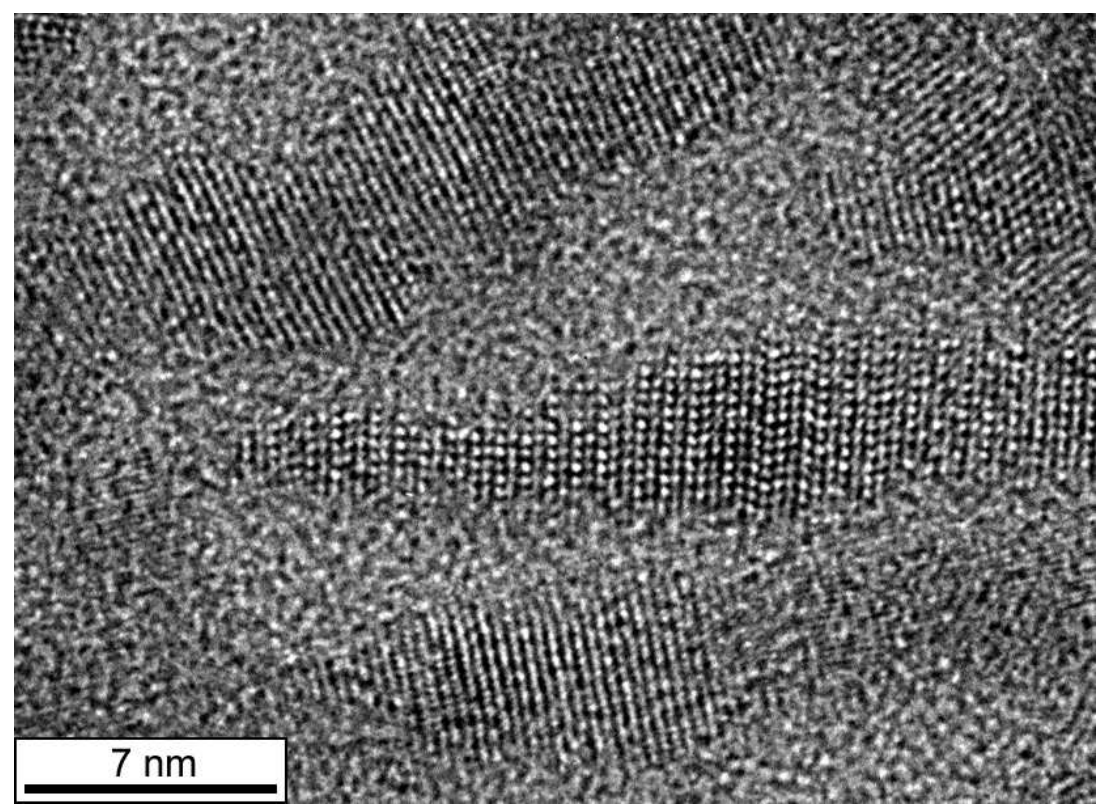

Figure 2.4: HR-TEM micrograph of CdSe nanorods.

According to the HR-TEM analysis, the nanorods grow along the CdSe c-axis and retain the symmetry of the wurtzite lattice, which renders them a hexagonal crossshape. The nanorods tend to have some stacking faults along the growth direction. The shape of the nanorods seems to be not completely cylindrical. A HR-TEM micrograph of a single nanorod is displayed in Figure 2.5.

The diameter of the nanorod varies along its length. Fluctuations of the nanorod diameter between the extreme values from $4-7 \mathrm{~nm}$ around the mean diameter of $6 \mathrm{~nm}$ can be observed along the nanorod. Most of the investigated nanorod samples show this behavior, the average diameter of the nanorods is still uniform throughout the samples. This is of importance for the properties of excitons in CdSe nanorods. Effectively, the excitons do not extend over the whole nanorod length but are weakly confined between diameter constrictions. This results in the excitonic fine structure being mostly determined by the diameter of the nanorod while being almost independent of the nanorod length[14]. For phonons, the diameter fluctuations are expected to lead to a broadening of diameter dependent quantities. 
Chapter 2. CdSe nanorods

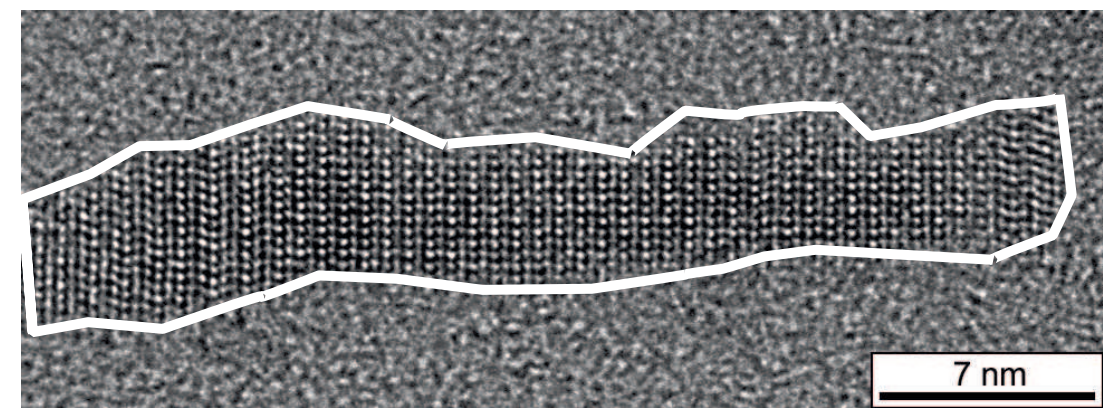

Figure 2.5: HR-TEM micrograph of a single CdSe nanorod. The nanorods' shape is highlighted. 


\subsection{Core-shell nanorods}

The nanorods can be coated with an epitaxial shell. To synthesize such nanocrystals made of more than one material, the formation of a large interface between the two materials can be achieved when the lattice constants of the two components do not differ significantly, this is the case with CdSe and ZnS, and when the control of the synthesis parameters keeps the interfacial energy. These prerequisites lead to the formation of core-shell type hybrid nanocrystals, in which the symmetry of the inner nanocrystal core is retained upon coverage by a layer of another material. The preparation technique aims to obtain selective heterogeneous nucleation of the shell onto preformed nanocrystal cores while suppressing the formation of separate nanocrystals of the second material. This requires a rather slow addition of the shell molecular precursors to the cores at relatively low temperatures.

The shell growth can be accomplished by several methods: by co-reacting all the necessary molecular precursors or by alternating deposition of monolayers of each atomic species that will compose the shell material, by replacement redox processes, in which the outermost layer of the core is converted into the shell material upon reaction with suitable reagents and by thermally-induced annealing of an initially amorphous or discontinuous shell. The different growth processes are visualized in Figure 2.6.

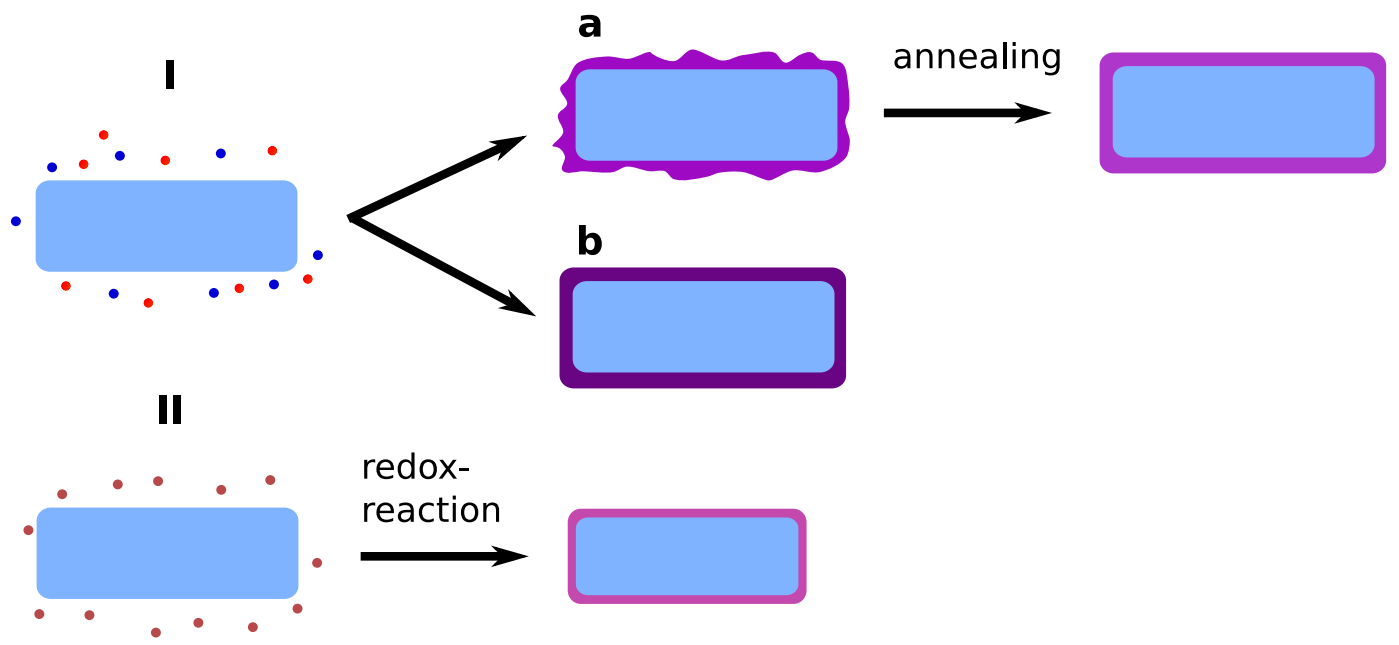

Figure 2.6: Shell growth schemes. The precursors co-react and form the shell or are deposited as alternating monolayers (I). The shell is either crystalline (I b) or amorphous and can be annealed to create a crystalline shell (I a). A redox process forms the shell out of the outermost core layer and precursors (II). 
The shell-growth process can be repeated with different precursors to create further shells of different materials, effectively onion-like multishell structures [34, 35] or even more sophisticated heterostructures[13, 17]. The shell can usually not grow thicker than a few monolayers without developing structural defects. To prevent this, buffer layers are grown. This strategy is used to coat the CdSe nanorods, where the growth of a CdS-ZnS shell of graded or mixed composition allows the strain to be released gradually.

The core-shell nanorods that are discussed in Chapter 7 consist of a CdSe nanorod core and a ZnS shell. The shell is synthesized by co-reacting diethylzinc as Zn precursor and thiourea as $\mathrm{S}$ precursor in a hexadecylamine/trioctylphosphine oxide reaction mixture at $\left(180^{\circ} \mathrm{C}\right)$. The thickness of the shell is controlled by the amount of added precursors.

To investigate the influence of the shell various core-shell nanorods are prepared. For each sample a fraction of bare nanorods is retained to allow a comparison of the Raman signal from core-shell nanorods with the Raman signal from the bare counterpart. The shell can hardly be resolved in HR-TEM measurements. The limited contrast in TEM between CdSe and ZnS makes a distinction between the core and the shell difficult. The core-shell nanorods appear mostly just thicker in the HRTEM micrographs. TEM is used to neglect the formation of individual nanocrystals from the shell precursors and the energy dispersive X-ray spectrum (EDX) can be used for a rough characterization. Figure 2.7 displays an EDX spectrum from coreshell nanorods.

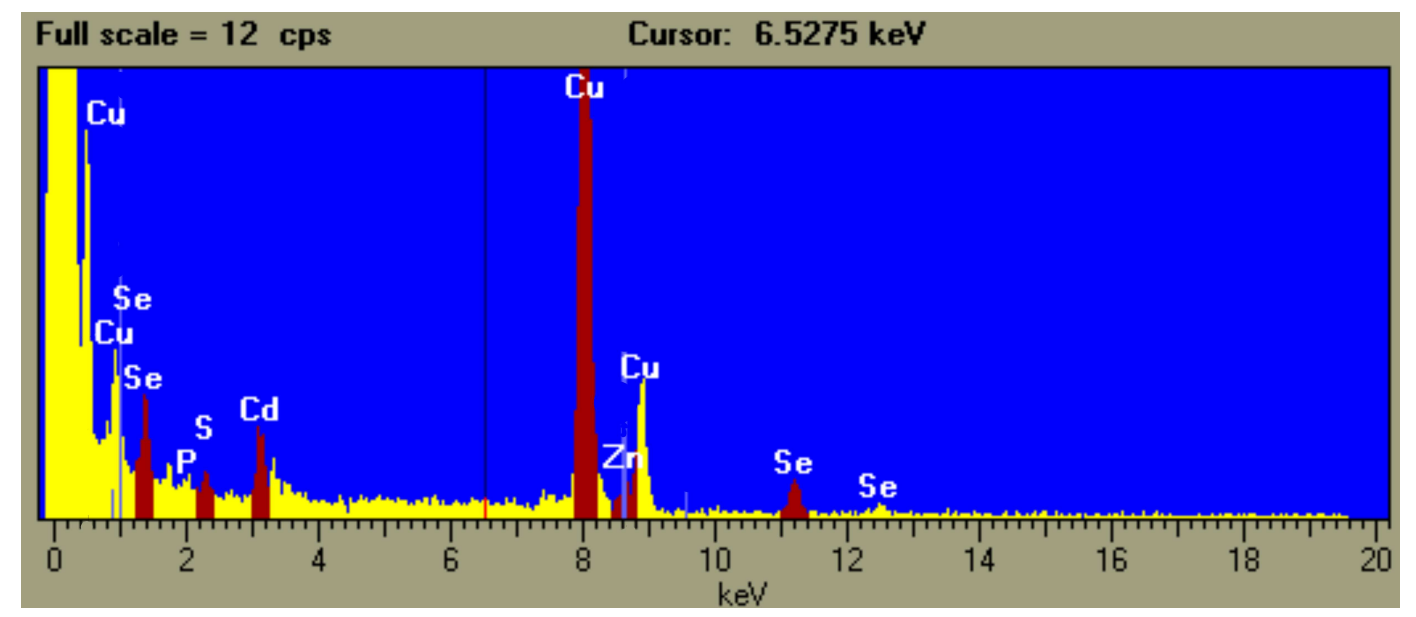

Figure 2.7: EDX spectrum of a CdSe-ZnS core-shell nanorod ensemble. EDX measurement by Tore Niermann.

$\mathrm{Cd}$ and Se are present in equal amounts. Zn and $\mathrm{S}$ are present in lower amounts, their relative amounts are as expected from the growth parameters. The C,P and $\mathrm{Cu}$ signals can be attributed to the supporting TEM-grid. 


\subsubsection{Luminescence properties}

The optical properties of core-shell CdSe-ZnS nanorods can be investigated by lowtemperature photoluminescence (PL) spectroscopy. Le Thomas et al. performed such measurements[36]. Figure 2.8 displays an emission spectrum of a single-nanorod with a 2-3 monolayer ZnS shell.

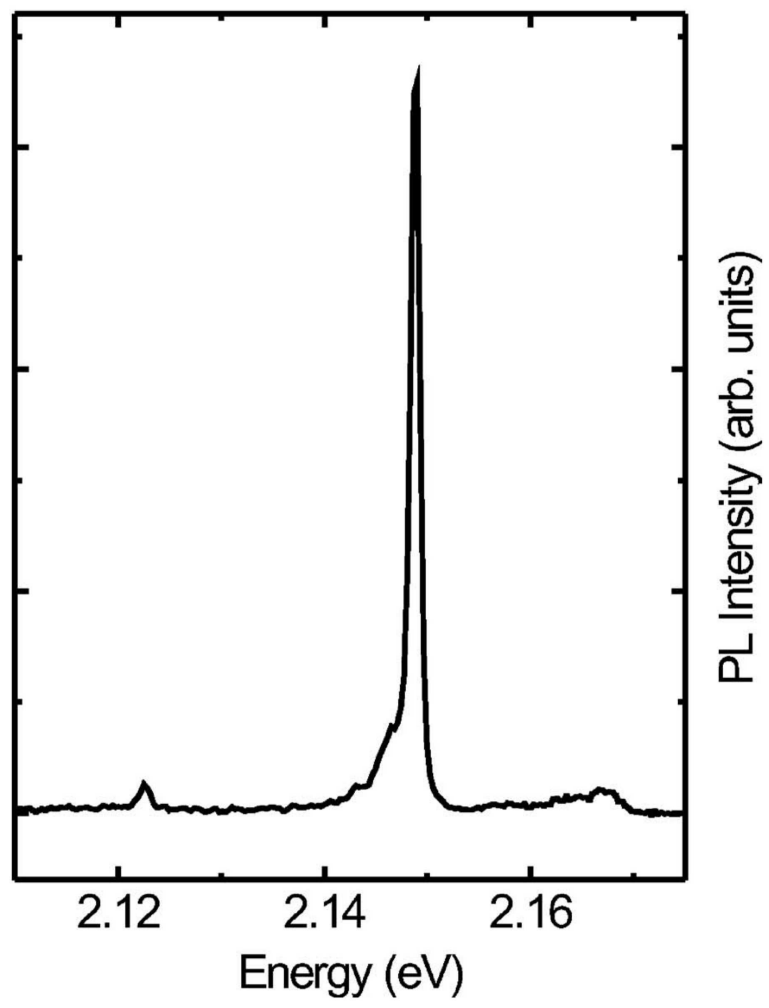

Figure 2.8: PL spectrum of a single CdSe nanorod with $5 \mathrm{~nm}$ diameter and $25 \mathrm{~nm}$ length at $T=10 \mathrm{~K}$. Taken from reference [37].

The PL spectrum is composed of a main peak, with a low-energy shoulder, and a redshifted peak by $28.4 \mathrm{meV}$ with respect to the main peak. The main peak and its low-energy shoulder can be attributed to the fourfold degenerate "light hole" exciton ground state. It is splitted into a degenerate state with total angular momentum projection along the rod axis \pm 1 , these constitute the low-energy shoulder and two other states with total angular momenta projection 0 , that form the main peak. The energy of the redshifted peak corresponds to the energy of the main peak reduced by the LO-phonon energy in bulk CdSe. This peak is thus the first LO-phonon replica 
Chapter 2. CdSe nanorods

of the exciton[36].

The ZnS shell has a significant impact on the photoluminescence from CdSe nanorods. Mokari and Banin synthesized CdSe nanorods with increasing shell sizes and measured their photoluminescence[20]. Figure 2.9 display absorption and PL spectra of CdSe nanorods with $4 \mathrm{~nm}$ diameter and $22 \mathrm{~nm}$ length and different shell sizes.

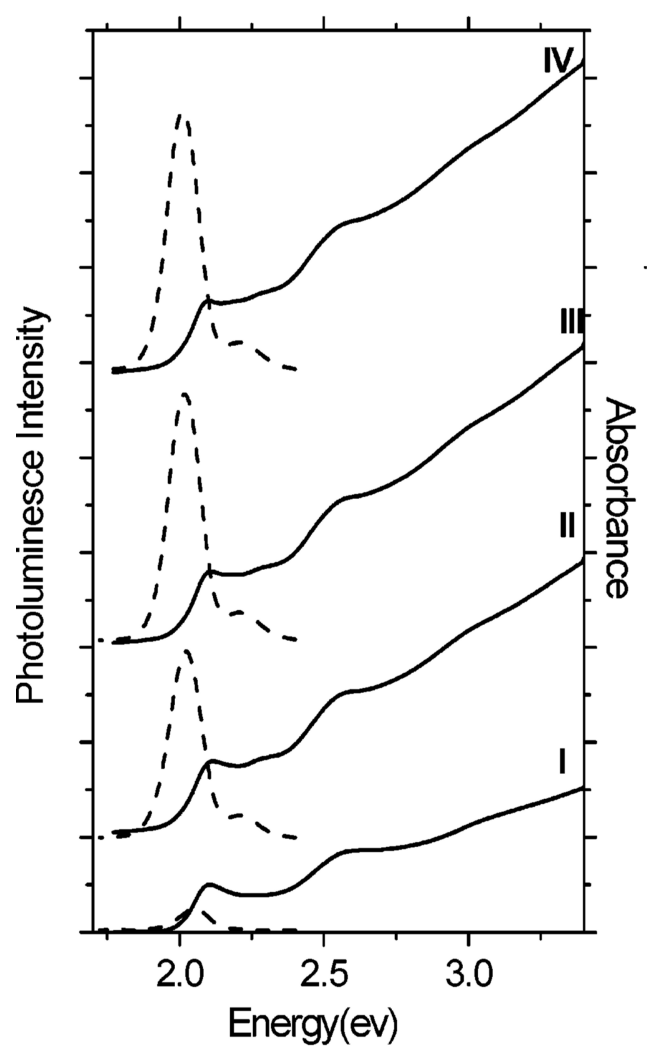

Figure 2.9: Absorption (solid lines) and photoluminescence (dashed lines) for increasing shell sizes (spectrum I corresponds to the core nanorod, IV is a 2 monolayer shell). Taken from reference [20].

Upon shell growth the peak position of the PL shifts slightly to lower wavelengths. The quantum yield is increased from $3 \%$ of the bare nanorods to $28 \%$ after adding a ZnS shell of 2 monolayer thickness.

The quantum yield increases and reaches a level of $28 \%$, an order of magnitude increase compared with the bare nanorods. Bryant and Jaskolski explain this by surface states[38]. They have used a tight-binding approach to study the effect of surface states on capped and uncapped nanocrystals. They find a broad band of mixed surface/internal states above the conduction band edge when the surface 


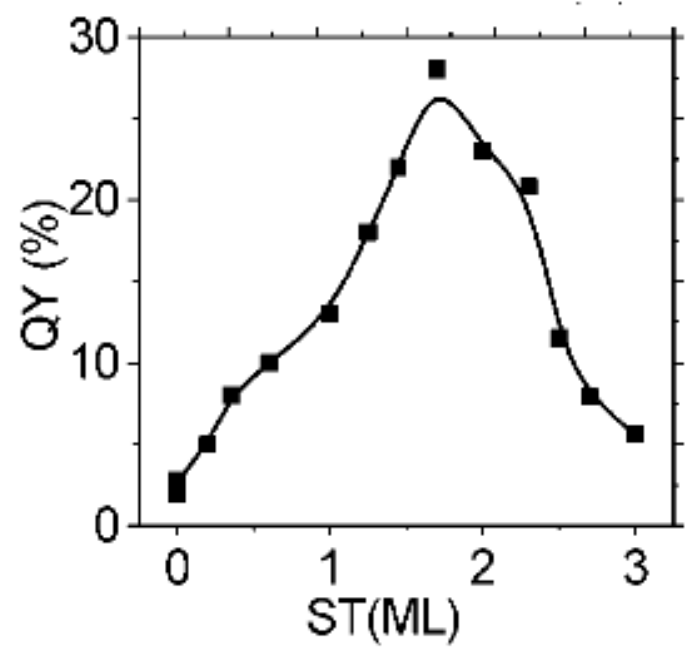

Figure 2.10: Quantum yield (QY) as a function of the shell thickness (ST). The QY increases for increasing ST until a critical thickness is reached, where strain leads to a reduction of the QY. Image taken from reference [20].

is unpassivated. This effects can be eliminated by capping the nanocrystal with a higher band gap shell. When a few monolayer cap is used, the effect of surface states decreases but is still significant. A thicker shell then fully suppresses the effects of the surface states on the internally confined states and the maximum quantum yield can be achieved[38].

A Further shell growth after reaching the maximum quantum yield leads to reduction of the quantum yield. Mokari and Banin attribute this to the large lattice mismatch of $11 \%$ between the core and the shell materials. In a thin shell the strain is accommodated. Further shell growth then leads to defect formation[20]. This can be seen in Figure 2.10, where the dependence of the quantum yield on the shell thickness is displayed. A similar trend can also be observed for other material systems like CdSe covered with ZnSe[39]. 



\section{Chapter 3}

\section{Methods}

Direct band-gap semiconductor nanocrystals like CdSe nanorods are investigated intensely by photoluminescence measurements[40-43]. This is due to the fact that their optical properties are crucial for many applications. For example, time-resolved photoluminescence spectroscopy can be used to study the electronic energy transfer in close-packed CdSe nanocrystal ensembles. The quenching of the luminescence lifetime with decreasing size accompanied by an enhancement of the luminescence for large crystals can be observed this way[44]. Photoluminescence excitation and fluorescence line narrowing spectroscopies can be used to examine the exciton fine structure[45]. Magnetophotoluminescence spectroscopy is applied to examine the influence of the nanocrystal asymmetry on the emission of single threedimensionally confined biexcitons, where the impact of electron-hole exchange interaction on the fine structure and the polarization properties of optical transitions can be revealed[46].

The structural properties of colloidal nanocrystals are mostly examined by HR-TEM and related methods. However, CdSe nanorods and especially core-shell nanorods are very challenging to investigate by HR-TEM, as mentioned in Chapter 2.

Details about the structural and vibrational properties of semiconductor nanostructures are often obtained from X-ray scattering experiments like X-ray crystallography, small angle X-ray scattering and inelastic X-ray scattering. However, X-ray scattering experiments usually require sufficiently large volumes of ordered crystals, bulk single crystals or ordered arrays of nanocrystals. Otherwise the diffraction patterns are too blurred to extract the crystallographic data. Thus, structural information from CdSe nanorods can not easily be obtained from X-ray diffraction measurements, as these structures can not be synthesized into sufficiently ordered arrays. Recently, there have been impressive advances in the exploitation of self-ordering processes in order to create large, ordered arrays of colloidal nanocrystals[47, 48]. 
Chapter 3. Methods

However, the crystal axes are still not sufficiently aligned to extract the lattice parameters from the X-ray diffraction patterns with adequate accuracy to gain information on strain etc.

Another popular method to determine the structural characteristics from crystalline solids, neutron scattering, is also not possible. The strong neutron absorbing nature of $\mathrm{Cd}$ forbids such experiments. The phonon dispersion of bulk CdSe was measured by inelastic neutron scattering on the very weakly neutron absorbing isotope ${ }^{116} \mathrm{Cd}$ and natural selenium[49]. But creating precursors with sufficient amounts of this CdSe isotope is extremely complex.

Raman spectroscopy and concomitant ab-initio calculations allow to gain access to structural information that is difficult to obtain with other methods. As the colloidal nanocrystals can be grown in very homogeneous ensembles with narrow size distributions and neglectable contamination with other particles, ensemble rather than single-nanocrystal measurements can be applied.

\subsection{Phonons in crystalline semiconductors}

Phonons are important for many physical properties of crystalline solids. They e.g. govern the thermal[50] and electrical conductivities[51] and they affect the optical properties due to interactions with excited charge carriers.

A phonon is a quantized mode of vibration of the crystal lattice. The ions of the crystal displace from their equilibrium position $\mathbf{R}$ in the Bravais lattice. The displacements from the equilibrium positions are usually small compared to the interionic spacing. The basic theory describing the dynamic behavior of lattice vibrations is called harmonic approximation. The position of each ion at the mean position $\mathbf{R}$ is $\mathbf{r}(\mathbf{R})=\mathbf{R}+\mathbf{u}(\mathbf{R})$, where the deviation from the mean position $\mathbf{u}(\mathbf{R})$ can be time-dependent. A pair of atoms separated by $\mathbf{r}$ contributes an amount $\phi(\mathbf{r})$ to the potential energy of the crystal. The total potential energy of the crystal is

$$
U=\frac{1}{2} \sum_{\mathbf{R} \mathbf{R}^{\prime}} \phi\left(\mathbf{r}(\mathbf{R})-\mathbf{r}\left(\mathbf{R}^{\prime}\right)\right)=\frac{1}{2} \sum_{\mathbf{R} \mathbf{R}^{\prime}} \phi\left(\mathbf{R}-\mathbf{R}^{\prime}+\mathbf{u}(\mathbf{R})-\mathbf{u}\left(\mathbf{R}^{\prime}\right)\right) .
$$

The dynamical problem is then governed by the Hamiltonian

$$
H=\sum_{\mathbf{R}} \frac{\mathbf{P}(\mathbf{R})^{2}}{2 M}+U
$$

where $\mathbf{P}(\mathbf{R})$ is the momentum of the atom at the equilibrium position $\mathbf{R}$, and $\mathrm{M}$ the atomic mass. 
For the harmonic approximation one expects the deviations from the equilibrium positions to be small. One can then use the three-dimensional form of the Taylor theorem to expand the potential energy $U$ about its equilibrium value. When retaining only the first nonvanishing correction to the equilibrium potential energy, the potential energy is written as

$$
U=U^{\text {eq }}+U^{\text {harm }}
$$

where $U^{\mathrm{eq}}$ is the equilibrium potential energy and

$$
\begin{aligned}
U^{\text {harm }} & =\frac{1}{4} \sum_{\mu, \nu=x, y, z}^{\mathbf{R R}^{\prime}}\left[\mu_{\mu}(\mathbf{R})-\mu_{\mu}\left(\mathbf{R}^{\prime}\right)\right] \phi_{\mu \nu}\left(\mathbf{R}-\mathbf{R}^{\prime}\right)\left[\mu_{\nu}(\mathbf{R})-\mu_{\nu}\left(\mathbf{R}^{\prime}\right)\right] \\
\phi_{\mu \nu}(\mathbf{r}) & =\frac{\partial^{2} \phi(\mathbf{r})}{\partial r_{\mu} \partial r_{\nu}} .
\end{aligned}
$$

Usually the equilibrium potential is ignored, as it is just a constant. The harmonic potentials are then treated as the total potential energy.

In a matrix notation (see ref.[52]) the harmonic potential can be written as

$$
U^{\text {harm }}=\frac{1}{2} \sum_{\mathbf{R R}^{\prime}} \mathbf{u}(\mathbf{R}) \mathbf{D}\left(\mathbf{R}-\mathbf{R}^{\prime}\right) \mathbf{u}\left(\mathbf{R}^{\prime}\right) .
$$

By considering the independence of the order of differentiation for the potential energy, the inversion symmetry of the Bravais lattice and by applying Born-von Karman periodic boundary conditions and plane waves $\mathcal{E} e^{i(\mathbf{k} \cdot \mathbf{R}-\omega t)}$ as eigenfunctions, the corresponding $3 N$ equations of motion ( $N$ is the number of ions) reduce to the eigenvalue problem[52]

$$
M \omega^{2} \mathcal{E}=\mathbf{D}(\mathbf{k}) \mathcal{E} .
$$

$\mathcal{E}$ is an eigenvector that describes the direction in which the ions move and $\mathbf{D}(\mathbf{k})$ is the dynamical matrix

$$
\mathbf{D}(\mathbf{k})=\sum_{\mathbf{R}} \mathbf{D}(\mathbf{R}) e^{-i \mathbf{k} \cdot \mathbf{R}}
$$

Applying fundamental matrix algebra results in the polarizations vectors $\mathcal{E}_{s}(\mathbf{k})$ and frequencies $\omega_{s}(\mathbf{k})$. For each value of $k$ there are $3 p$ normal modes. $p$ is the number of ions in the basis. The frequencies $\omega_{s}(\mathbf{k})(s=1, \cdots, 3 p)$ are all functions of $\mathbf{k}$, with the periodicity of the crystal lattice. Three of the $3 p$ branches are acoustic, they describe vibrations that vanish in the long-wavelength limit. The other modes are optical. In case of polar materials, like CdSe, they correspond to a mode of vibration where positive and negative ions vibrate against each other. This can create an electrical dipole moment, effectively allowing the coupling to an electric field, e.g. light.

In analogy with the quantum theory of the electromagnetic field, where photons are 
Chapter 3. Methods

the quanta of the radiation field, the vibrational modes can be described as phonons. The mode of a branch $s$ with wave vector $\mathbf{k}$ in its $n_{\mathbf{k} s}$ th excited state can be written as $n_{\mathbf{k} s}$ phonons of type $s$.

A more detailed discussion of the formalism presented above can be found in ref. [52].

Optical phonons can be treated numerically by ab-initio calculations and, especially in polar materials, accessed experimentally via Raman spectroscopy. These methods will be presented below.

\subsection{Density functional theory, ab-initio calcula- tions}

The Density Functional Theory (DFT) is an alternative approach to the use of wave functions in the theory of electronic structure. The calculations are called ab-initio, or first-principles calculations if only fundamental constants of physics rather than fit results are utilized. The theory and the first computational implementations were mainly developed by Walter Kohn and John Pople and awarded with the Nobel price in Chemistry in 1998. Walter Kohn "for his development of the density-functional theory" and John Pople "for his development of computational methods in quantum chemistry" [53, 54].

The foundation of the theory of electronic structure of matter is the nonrelativistic Schrödinger equation for the many-electron wave function $\Psi$,

$$
\left\{-\frac{\hbar^{2}}{2 m} \sum_{j} \nabla_{j}^{2}-\sum_{j, l} \frac{Z_{l} e^{2}}{\left|r_{j}-R_{l}\right|}+\frac{1}{2} \sum_{j \neq j^{\prime}} \frac{e^{2}}{\left|r_{j}-r_{j^{\prime}}\right|}-E\right\} \Psi=0 .
$$

$r_{j}$ are the positions of the electrons, $R_{l}$ and $Z_{l}$ are the positions and atomic numbers of the nuclei. The wave function $\Psi$ depends on the positions and spins of the $N$ electrons. The electronic properties depend parametrically on $R_{l}$ and in particular the electronic density $n(r)$ and total energy $E$, implying the Born-Oppenheimer approximation, in which the electrons are considered to follow the movement of the heavy nuclei instantaneously. For a system of atoms with a total of $N$ interacting electrons, with the influence of symmetries and spin ignored, the number $M$ of parameters required to find the many-electron wave function is $M=p^{3 N}$, were $3 \leq p \leq 10$ is an approximated number of parameters per variable needed for an accurate result. For $N=100$ electrons this would require a quantity in a space of $M \approx 10^{150}$ dimensions to be minimized. This is impossible with current computer systems. Real, physical crystals contain more than $10^{21}$ electrons, which makes a closed-form solution practically impossible. 
The approach of DFT is to focus on quantities in the real, three-dimensional coordinate space. Mainly, on the electron density $n(r)$.

In 1964 Hohenberg and Kohn demonstrated that the ground-state density $n(r)$ of a bound system of interacting electrons in an external potential $v(r)$ determines this potential uniquely. This is mathematically rigorous. Following the HohenbergKohn variational principle, the energy of the electron ground state can be obtained by minimizing

$$
E=\min _{\bar{n}(r)}\left\{\int v(r) \bar{n}(r) d r+F[\bar{n}(r)]\right\},
$$

where $F[\bar{n}(r)]=\min _{\alpha}\left[\Psi_{\bar{n}(r)}^{\alpha},(T+U) \Psi_{\bar{n}(r)}^{\alpha}\right] \cdot \bar{n}(r)$ is a trial electronic density and $\Psi_{\bar{n}(r)}^{\alpha}$ the class of trial functions with this $\bar{n}(r) .(T+U)$ is the sum of the kinetic and the interaction energy.

The problem of finding the minimum of $(\bar{\Psi}, H, \bar{\Psi})$ with respect to the $3 N$-dimensional trial function $\bar{\Psi}$ in wave functions has been transformed into the problem of finding the minimum of $E_{\nu}[\bar{n}(r)]$ with respect to the three-dimensional trial function $\bar{n}(r)$.

In 1965 Kohn and Shan formulated an alternative expression for $F[\bar{n}(r)]$ for interacting electrons[55], namely

$$
F[\bar{n}(r)] \equiv T_{S}[\bar{n}(r)]+\frac{1}{2} \int \frac{\bar{n}(r) \bar{n}\left(r^{\prime}\right)}{\left|r-r^{\prime}\right|} d r d r^{\prime}+E_{x c}[\bar{n}(r)]
$$

where $T_{S}[\bar{n}(r)]$ is the kinetic energy functional for noninteracting electrons and $E_{x c}[\bar{n}(r)]$ is the exchange-correlation energy functional, where $E_{x c}[\bar{n}(r)]$ is generally not known and has to be defined by a suitable approximation.

Minimizing the density $n(r)$ is then performed by solving the equation

$$
\left(-\frac{1}{2} \nabla^{2}+v_{e f f}(r)-\epsilon_{j}\right) \varphi_{j}(r)=0
$$

with

$$
\begin{gathered}
n(r)=\sum_{j=1}\left|\varphi_{j}(r)\right|^{2}, \\
v_{e f f}=v(r)+\int \frac{n\left(r^{\prime}\right)}{\left|r-r^{\prime}\right|} d r^{\prime}+v_{x c}(r),
\end{gathered}
$$

with the local exchange-correlation potential $v_{x c} \cdot \varphi_{j}(r)$ are called Kohn-Sham wave functions. The ground state energy is given by

$$
E=\sum_{j} \epsilon_{j}+E_{x c}[n(r)]-\int v_{x c}(r) n(r) d v-\frac{1}{2} \frac{n(r) n\left(r^{\prime}\right)}{\left|r-r^{\prime}\right|} .
$$


Chapter 3. Methods

In principle, with the exact $E_{x c}$ and $v_{x c}$, all many-body effects would be included.

The practical usefulness of ground-state DFT depends entirely on whether approximations for the functional $E_{x c}[\bar{n}(r)]$ can be found which are at the same time sufficiently simple and sufficiently accurate. A frequently used approximation for $E_{x c}[\bar{n}(r)]$ is the so-called local-density approximation (LDA).

$$
E_{x c}^{L D A}=e_{x c}(n(r)) n(r) d r
$$

with $e_{x c}$ as exchange-correlation energy per particle of a uniform electron gas of density $n[53]$. The exchange part is elementary and given, in atomic units, by

$$
e_{x}=\frac{0.458}{r_{s}}
$$

where $r_{s}$, is the radius of a sphere containing one electron. The correlation part is

$$
e_{c}=\frac{0.44}{r_{s}+7.8}
$$

LDA usually gives ionization energies of atoms, dissociation energies of molecules and cohesive energies with only a fair accuracy compared to the experimental values of typically $10-20 \%$. However the LDA gives bond lengths and thus the geometries typically with a reasonable accuracy of 1\%. LDA is the underlying basis of many approximations in use in DFT. Technical details vary depending on the problem and the computational framework.

\subsubsection{SIESTA}

The SIESTA method, acronym for Spanish Initiative for the Electronic Simulations of Thousands of Atoms, is an implementation of the density functional theory[56]. An accessible introduction to SIESTA and its usage can be found in reference [57]. SIESTA implements a fully selfconsistent DFT, based on a flexible linear combination of atomic orbitals (LCAO) as basis set. That means the required computer resources grow linearly when increasing the number $N$ of atoms in a simulation box. It allows fast, converged simulations using minimal basis sets and accurate calculations with complete multiple zeta and polarized bases, depending on the required accuracy and available computational power. Various methods are implemented in the SIESTA code to generate these numerical basis sets. The approximations concern the treatment of exchange and correlation, and the use of pseudopotentials. With the SIESTA method, exchange and correlation are treated within the 
Kohn-Sham DFT. It allows for both the local (spin) density approximation and the generalized gradient approximation. Standard norm-conserving pseudopotentials are used in their fully nonlocal form. SIESTA reads the norm-conserving pseudopotentials in a semilocal form (a different radial potential $V_{l}(r)$ for each angular momentum $l$ ) from a data file that users can fill with their preferred choice. Generally the Troullier-Martins parametrization is used.

The numerical parameters must be converged to the desired accuracy before the actual calculation can be performed. During a calculation the Hamiltonian and overlap matrices are built and stored, and the Kohn-Sham equations are solved by a self-consistent loop. The starting point is an electron density obtained from the sum of all atomic electronic densities. Then the Hamiltonian is built and diagonalized and a new density is found. This process is repeated until the change in density is lower than a certain threshold. In a standard calculation the total energy per unit cell is obtained, as well as the forces on all atoms, the stress, pressure, etc. The program also allows to calculate phonon frequencies. There are other calculation modalities implemented, which yield for example the band structure (Kohn-Sham eigenvalues), wave functions (eigenvectors), or relax the geometry with a conjugate-gradients algorithm. The geometry relaxation may be restricted, to impose certain positions or coordinates, or with more complex constraints. It is also possible to perform a variety of molecular dynamics simulations, at constant energy or temperature, and at constant volume or pressure.

\subsubsection{Ab-initio calculations of bulk CdSe and CdSe nanorods}

To estimate the properties of CdSe nanorods and to allow a better understanding of the experimental findings, the results from ab-initio calculations are used. All calculation results and images are courtesy of Marcel $\operatorname{Mohr}^{1}[58,59]$.

\section{CdSe phonon dispersion}

The phonon dispersion of bulk CdSe is not available from experiments with sufficient accuracy for an exertion in numerical models that e.g. treat confined phonons or heat transfer. For the direction $\Gamma-A$ ab initio calculations, a shell-model and inelastic neutron scattering data are available[49]. The inelastic neutron scattering measurements were performed with a crystal containing the weak neutron absorbing

\footnotetext{
${ }^{1}$ Present affiliation: Institut für Festkörperphysik, Technische Universität Berlin.
} 
Chapter 3. Methods

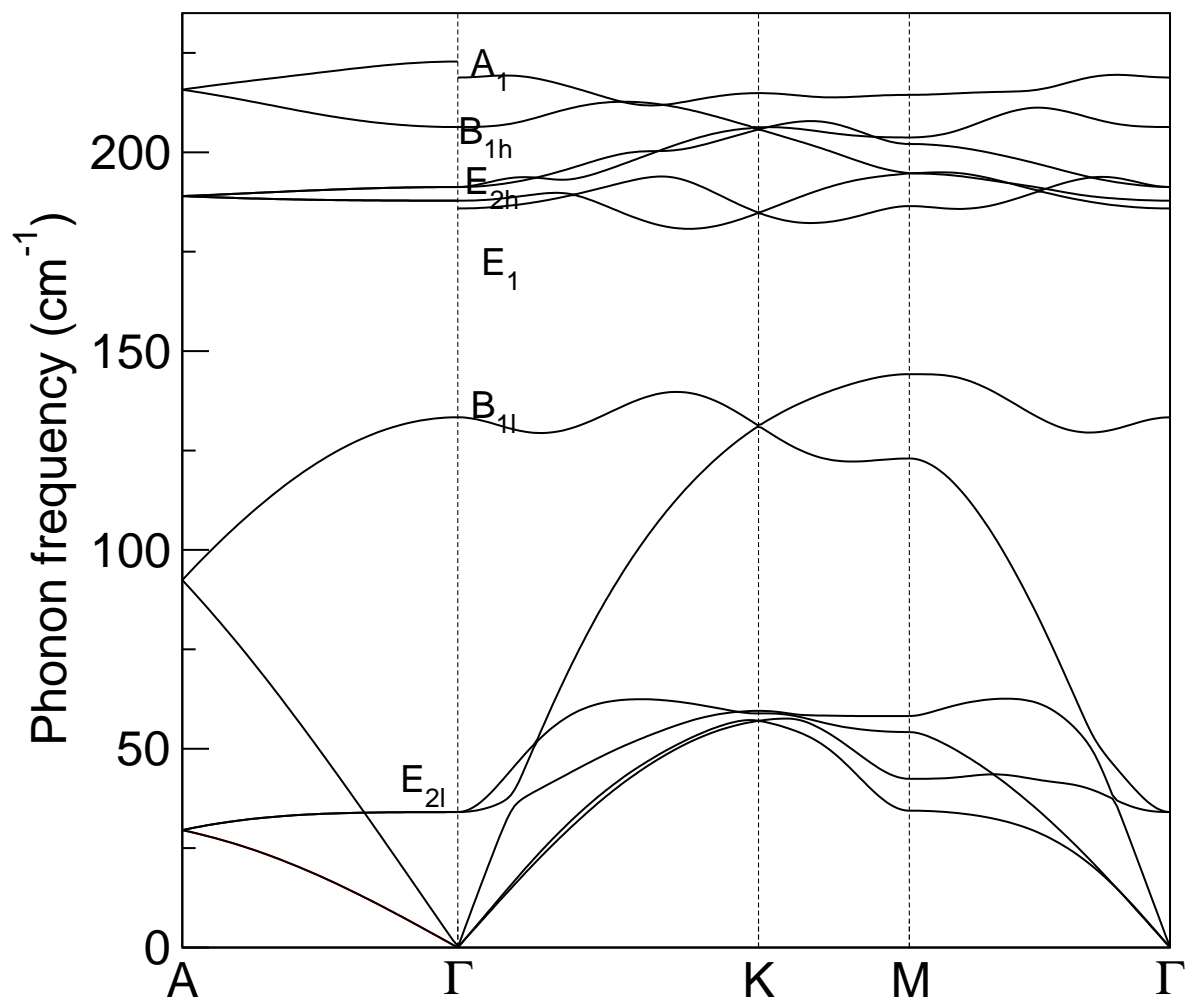

Figure 3.1: Phonon dispersion of CdSe. Calculations by Marcel Mohr[58].

isotope ${ }^{116} \mathrm{Cd}$. But no experimental data on the phonon dispersion is available for wave vectors $q$ in the basal plane. The complete phonon dispersion of CdSe can be calculated with a DFT-based linear response approach, which is implemented in the ABINIT package[60]. A calculated CdSe phonon dispersion by Mohr and Thomsen can be found in reference [58]. The authors use the exchange correlation functionals of Perdew and Wang in the local-density approximation[61]. The resulting phonon dispersion is displayed in Figure 3.1. All calculated frequencies are multiplied by a constant factor in order to match the experimental bulk longitudinal optical phonon (LO) frequency at the $\Gamma$-point. The shape of the dispersion along $\Gamma-K$ and $\Gamma-M$ will become of importance when treating confined phonons with a zone-folding approach as done in Chapter 4 . When the phonons become confined, phonons close to the $\Gamma$-point also contribute to the Raman spectrum. The slope near the $\Gamma$-point then determines the direction and amount of the shift of the phonon frequency with changing crystal dimensions. 


\section{CdSe nanorods}

Many properties of CdSe nanorods can be calculated with the SIESTA code. Mohr et al. performed such calculations [58, 59].

Mohr et al. use the local density approximation of the density functional theory with atom-centered confined numerical basis functions as implemented in the SIESTA code. The nanorod geometries for the SIESTA framework are created on the basis of wurtzite-structured CdSe nanocrystals with a cylindrical shape. As the aspect ratios of the physical nanorods are large, they can be approximated as wires of infinite length. For the investigation of the effects of an epitaxial $\mathrm{ZnS}$ shell a $\mathrm{Zn}$ and $\mathrm{S}$ atom can initially be placed on the Cd and Se sites, respectively.

Mohr et al. generate norm-conserving pseudopotentials with the Troullier Martins scheme. In their calculations, the valence electrons are described by a double- $\zeta$ basis set plus an additional polarizing orbital. The nanorods are relaxed until all forces are below $0.02 \mathrm{eV} / \AA$.

The calculations are validated with a comparison between calculated and experimental values of constants: Values of 55.5 and $80.2 \mathrm{GPa}$ for the bulk modulus are obtained from the calculations for CdSe and ZnS, respectively, in agreement with the experimental values of 53.4 and $76.0 \mathrm{GPa}$ [62]. The lattice parameters for wurtzite CdSe $a=4.34$ Aand $c=7.09 \AA$ are also in good agreement with experimental values[28].

Symmetry considerations allow the reduction of the calculation time: Finite-differences phonon calculations usually scale by the order of 4 with the number of atoms in the unit cell. However, all one-dimensional systems can be described in terms of line groups, a one-dimensional analogon to space groups. In contrast to the latter line groups allow the application of arbitrary rotations. The force constants can then be calculated for the irreducible part of the unit cell. For the remaining atoms, the force constants can be determined by symmetry. Symmetry considerations also help to reduce the internal stress in the unit cell that can occur in large systems during the relaxation process. Most routines only regard atomic forces during relaxation, however stresses may appear by cancelling out of two or more forces. To prevent this a symmetry based, iterative relaxation method can be applied: After a nanorod structure is relaxed, the whole structure is generated again by applying symmetry operations to the irreducible part of the unit cell. This symmetry generated unit cell is then relaxed again. With this method better results than with a simple relaxation can be achieved: For example, the frequencies of phonons belonging to more-dimensional representations (that should have degenerate frequencies) are separated less. The size of the calculated nanorods is limited by their computational costs. The largest calculated nanorod takes about three weeks on a common four-unit cluster for the initial relaxation and two weeks for further relaxations con- 
a)

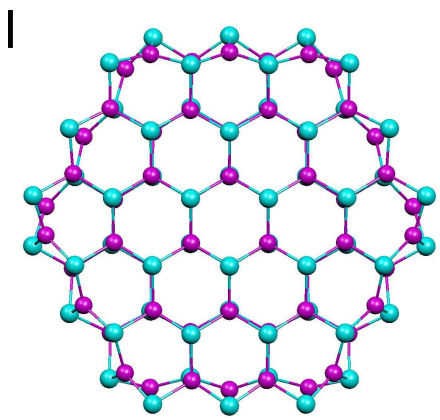

b)

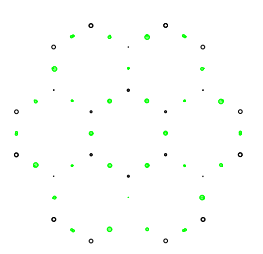

॥

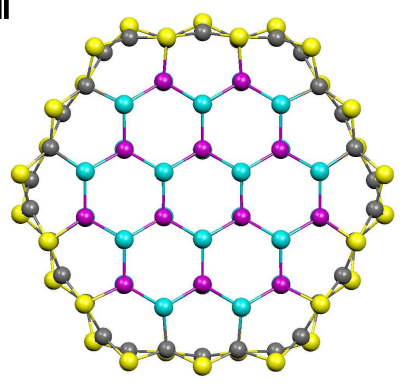

$3 \%$

○ $2 \%$

- $1 \%$

- $-1 \%$

(10) $-2 \%$

(10) $-3 \%$
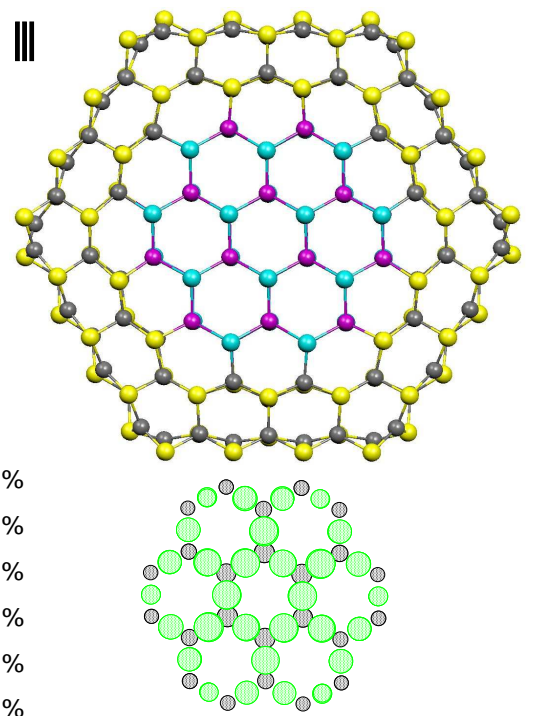

Figure 3.2: Cross sections of the CdSe NRs (a). The following color code is used: Cd (magenta), Se (cyan), Zn (black), S (yellow). Bond length deviations compared to bulk CdSe (b). Black corresponds to bonds parallel to the c-axis, green to all other bonds. Image courtesy of Marcel Mohr.

sidering symmetry. This nanorod has a diameter that is at the lower diameter limit of the available synthesized CdSe nanorods, which allows a direct comparison. Smaller structures are then calculated to estimate trends. Shells of various sizes are also included in the calculations.

The relaxed cross sections of the calculated CdSe nanorods are displayed in Figure 3.2 a). For a systematic study calculations on a bare CdSe nanorod (rod I) and two corresponding core-shell CdSe-ZnS nanorods (rod III and rod IV) are performed. To distinguish between the effects of the $\mathrm{ZnS}$ shell and size dependent effects, a larger bare CdSe NR (rod II) is calculated. It is of the same size than the core-shell nanorod with a single monolayer ZnS shell, rod III.

The bond lengths in the CdSe nanorods differ from the bond lengths in bulk CdSe. The bond lengths of the relaxed nanorods projected on the plane perpendicular to the c-axis are presented in Figure $3.2 \mathrm{~b}$ ). The bonds are centered in the middle of the atoms they connect. The radius of the circles is proportional to the deviation from the bulk CdSe bond length. Black circles correspond to bonds parallel to the c-axis, green circles correspond to all other bonds. For the bare CdSe nanorods, the most dramatic changes in the atomic structure happen in the outermost layer: the Cd-atoms rotate into the surface to lower the energy at the surface. This effect does not vanish if the surface is passivated with e.g. organic ligands. In concommi- 
tant calculations with a passivating hydrogen layer at the boundary no qualitative changes of this reconstruction are found[59]. This kind of surface reconstruction was first reported in Ref. [63]. When the diameter of the nanorod is increased, the strain due to the surface reconstruction decreases for the inner parts of the nanorod. In total, larger nanorods are less strained.

The coverage of a CdSe NR with a ZnS shell leads to a compressive strain of the CdSe core due to the small lattice parameter of $\mathrm{ZnS}$ compared to CdSe. The diameter $d$ of rod III is $\approx 5 \%$ smaller than that of rod II. All bonds of the CdSe core are shortened. The bonds in the lateral direction are much more strongly compressed than the bonds parallel to the c-axis. For the lateral bonds the average decrease is $3.5 \%$, while it is $1.5 \%$ for the bonds parallel to $c$. The maximal decrease is $4.5 \%$ and $3.6 \%$ for lateral and parallel bonds, respectively. The bonds parallel to the c-axis show the trend of being less compressed when lying closer to the surface. In contrast, the lateral bonds are homogeneously compressed throughout the CdSe core. This homogeneous compression can be approximated as uniaxial strain and is used te experimentally investigate strain in core-shell nanorods in Chapter 7. 
Chapter 3. Methods

\subsection{Raman spectroscopy}

Raman scattering is a non-destructive experimental method. It was first observed by Sir Chandrasekhara Venkata Raman and published in 1928[64, 65]. The great importance of this method for material science was soon recognized and Sir Venkata Raman was awarded the Nobel price in 1930 "for his work on the scattering of light and for the discovery of the effect named after him".

Raman spectroscopy is a very universal kind of spectroscopy. The light does not need to be absorbed by the sample (as it is for example necessary in fluorescence and photoluminescence spectroscopy) and its non-invasive nature and many possible combinations with different types of microscopies (confocal, etc) makes it quite versatile.

The Raman effect is the scattering of photons by matter, this can be molecules, crystalline solids or else, with a change of frequency.

The interaction between electromagnetic radiation and a crystal in full generality is very complex. In Raman scattering, however, the wavelength of the incident and scattered radiation is much greater than the size of the atoms and the interaction Hamiltonian can thus be written in terms of the polarizability P. For an incident photon of energy $\hbar \omega_{0}$, momentum $\mathbf{k}_{0}$, a scattered photon of energy $\hbar \omega_{s}$, and momentum $\mathbf{k}_{s}$, the crystals' initial state energy $E_{i}$ and the finals state energy $E_{f}$, the propability of the scattering can derived from second order perturbation theory as

$$
w=\frac{2 \pi}{\hbar} p\left(\omega_{s}\right)\left|H\left(\mathbf{k}_{0}, i: \mathbf{k}_{s}, f\right)\right|^{2} \delta\left(\hbar \omega_{0}+E_{i}-\hbar \omega_{s}-E_{f}\right),
$$

where $p\left(\omega_{s}\right)$ is the density of state of the photons, which is given by

$$
p\left(\omega_{s}\right) d \omega_{s} d \Omega=\left(\frac{1}{2 \pi c}\right)^{3} \frac{\omega_{s}^{2}}{\hbar} d \omega_{s} d \Omega .
$$

$d \Omega$ is an element of solid angle. The interaction between the photons and the crystal is specified by the Hamiltonian $H$. The interaction Hamiltonian is

$$
H=\sum_{\alpha \beta} P_{\alpha \beta}\left(\mathbf{k}_{0} i: \mathbf{k}_{s} f\right) E_{\alpha}(0) E_{\beta}(s),
$$

where $E(0)$ and $E(s)$ are the electric fields at the incident and scattered light and $\alpha$ and $\beta$ denote the Cartesian cooridnates. The electric field can be written in terms of photon creation and destruction operators. The intensity of the Raman scattering from state $i$ to state $f$ of the crystal then becomes

$$
\begin{aligned}
I d \Omega d \omega_{f} & =\frac{\omega_{f}^{4}}{2 \pi c^{3}} \sum_{\alpha \beta \gamma \delta} P_{\alpha \beta}\left(\mathbf{k}_{0} i: \mathbf{k}_{s} f\right) P_{\gamma \delta}^{+}\left(\mathbf{k}_{0} i: \mathbf{k}_{s} f\right) \cdots \\
& \cdots E_{\alpha}(0) E_{\gamma}(0) \varepsilon_{\beta}(s) \varepsilon_{\delta}(s) \delta\left(E_{i}+\hbar \omega_{0}-E_{f}-\hbar \omega_{s}\right)
\end{aligned}
$$


The total Raman scattering intensity can then be obtained by averaging over the states of the crystal and weighting them by thermodynamic factors. This leads to

$$
I d \Omega d \omega_{f}=\frac{\omega_{f}^{4}}{2 \pi c^{3}} \sum_{\alpha \beta \gamma \delta} E_{\alpha}(0) E_{\gamma}(0) \varepsilon_{\beta}(s) \varepsilon_{\delta}(s) I_{\alpha \beta \gamma \delta} .
$$

$I_{\alpha \beta \gamma \delta}$ is the Raman tensor.

Usually, the frequency of the incident photon is much greater than the frequency transfer. Raman scattering then occurs at the frequencies of the phonons whose wavevectors are equal to the wavevector transfer. As the incident light has a much longer wavelength than the interatomic distances and the available momentum transfer is much smaller than the momentum of the phonons, $|\mathbf{Q}|=0$ applies for the scattering. $\mathbf{Q}$ is the momentum transfer.

The incident light excites a virtual electron-hole pair, which either creates or absorbs a phonon und then recombines emitting the scattered photon.
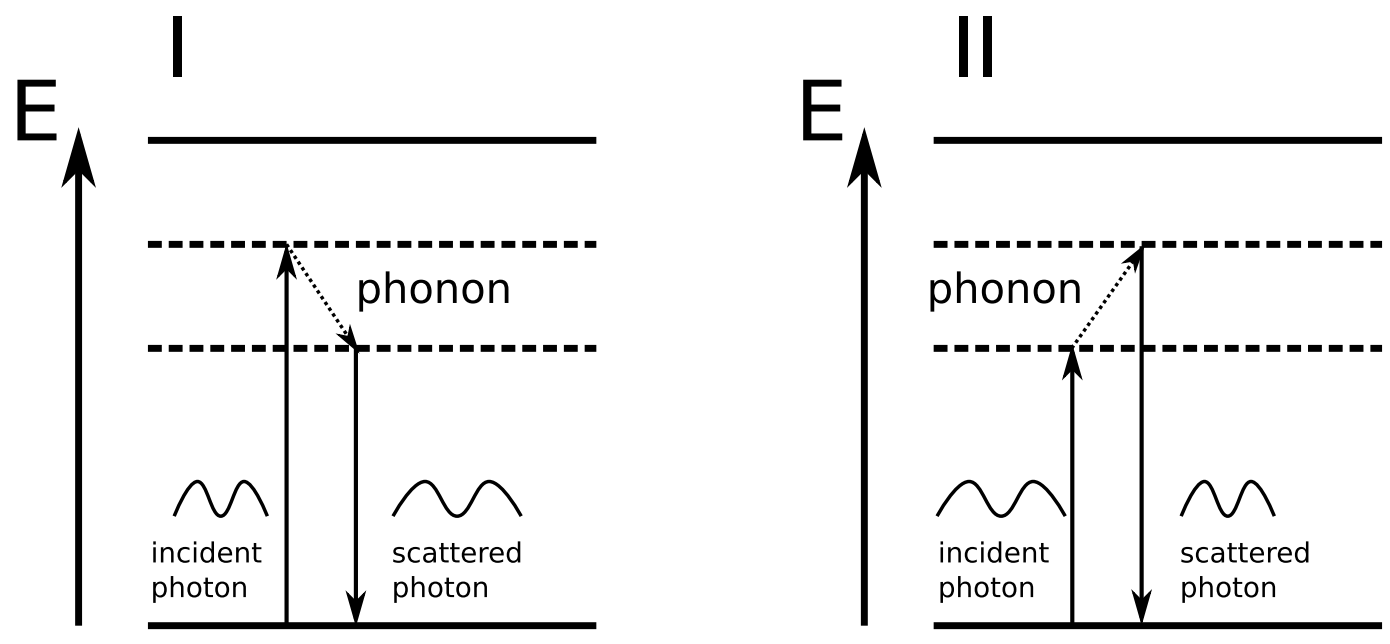

Figure 3.3: Scheme of a Stokes (I) and an anti-Stokes (II) Raman scattering process: An incident photon is absorbed, the excited carrier exchanges energy with the lattice by creating (I) or annihilating (II) a phonon and a photon is with changed energy is emitted; Solid lines represent real states, dashed lines correspond to virtual states.

In Figure 3.3, a scheme of the two fundamental Raman scattering processes is displayed. After an incident photon with the frequency $\omega_{L}$ is absorbed, the virtual electron-hole pair creates or annihilates a phonon with the frequency $\omega_{p h}$ (Figure 3.3 I and II respectively) and recombines, emitting a new photon with the frequency $\omega_{S}$. The energy difference between the incident and scattered photon $\hbar\left(\omega_{L} \pm \omega_{p h}\right)$ can be measured.

The Raman tensor in equation 3.22 has the full point symmetry of the crystal and is a fourth rank tensor. Following group theory, a phonon will only contribute to the 
Chapter 3. Methods

Raman scattering if it belongs to one of the irreducible representations present in the reduction of the Raman tensor. This leads to certain phonons being not Raman active and a dependence of the Raman scattering intensity on the polarization of the incident light and the orientation of the crystal.

Surface effects can contribute to the Raman scattering, in case of resonances greatly enhancing the Raman signal intensity. This can be exploited in nanoscience. For example, optical detection and spectroscopy of single molecules and single nanoparticles can be achieved with the use of surface-enhanced Raman scattering. Individual colloidal nanoparticles can be screened for size-dependent properties and then used to amplify the spectroscopic signatures of adsorbed molecules[66]. A fine metal tip brought to within a few nanometers of a molecular film gives a strong enhancement of Raman scattered light. This can be used for molecular analysis with the spatial resolution of an atomic force microscopy, as only limited by the tip size and shape. When scanning the tip over the sample surface, topographic information is obtained simultaneously and can be directly correlated with the spectroscopic data[67].

\subsubsection{Raman spectroscopy of CdSe nanorods}

There are several experimental requirements that have to be fulfilled for the Raman spectroscopy of solids. The Raman scattered light has a very low intensity relative to the intensity of the incident light. Thus a bright light source is required. It also has to be monochromatic with a narrow linewidth. Otherwise the elastically, Rayleigh scattered light outshines the Raman scattered light. The relative intensity of the Rayleigh scattered light is usually more than hundred times larger than Raman scattered light. A narrow-line continuous-wave laser fullfills these requirements. An $\mathrm{Ar}^{+}$-laser is used as excitation source. To measure the Raman signal a sensitive detector is required and the Rayleigh scattered light has to be suppressed. This can be accomplished by a charge coupled device camera (CCD) and two monochromators in subtractive mode, that function as a bandpass for the Raman signal. The experimental set-up for the measurements presented here is displayed in Figure 3.4.

$\mathrm{Ar}^{+}$has various transitions that allow a stimulated emission. Of these, the transition in the green part of the visible spectrum at $514.5308 \mathrm{~nm}$ is the most intense. There is no difference between the normalized Raman spectra of CdSe nanorods that are excited with blue $\mathrm{Ar}+$-lines and green $\mathrm{Ar}^{+}$-lines, so the $514.5308 \mathrm{~nm}$-line is used for all presented measurements. A small monochromator works as bandpass. $\mathrm{Ar}^{+}$plasma-lines, which are not resonantly amplified, can be present in the laser spectrum. These have wavelengths close to the laser line and can disturb the Raman 


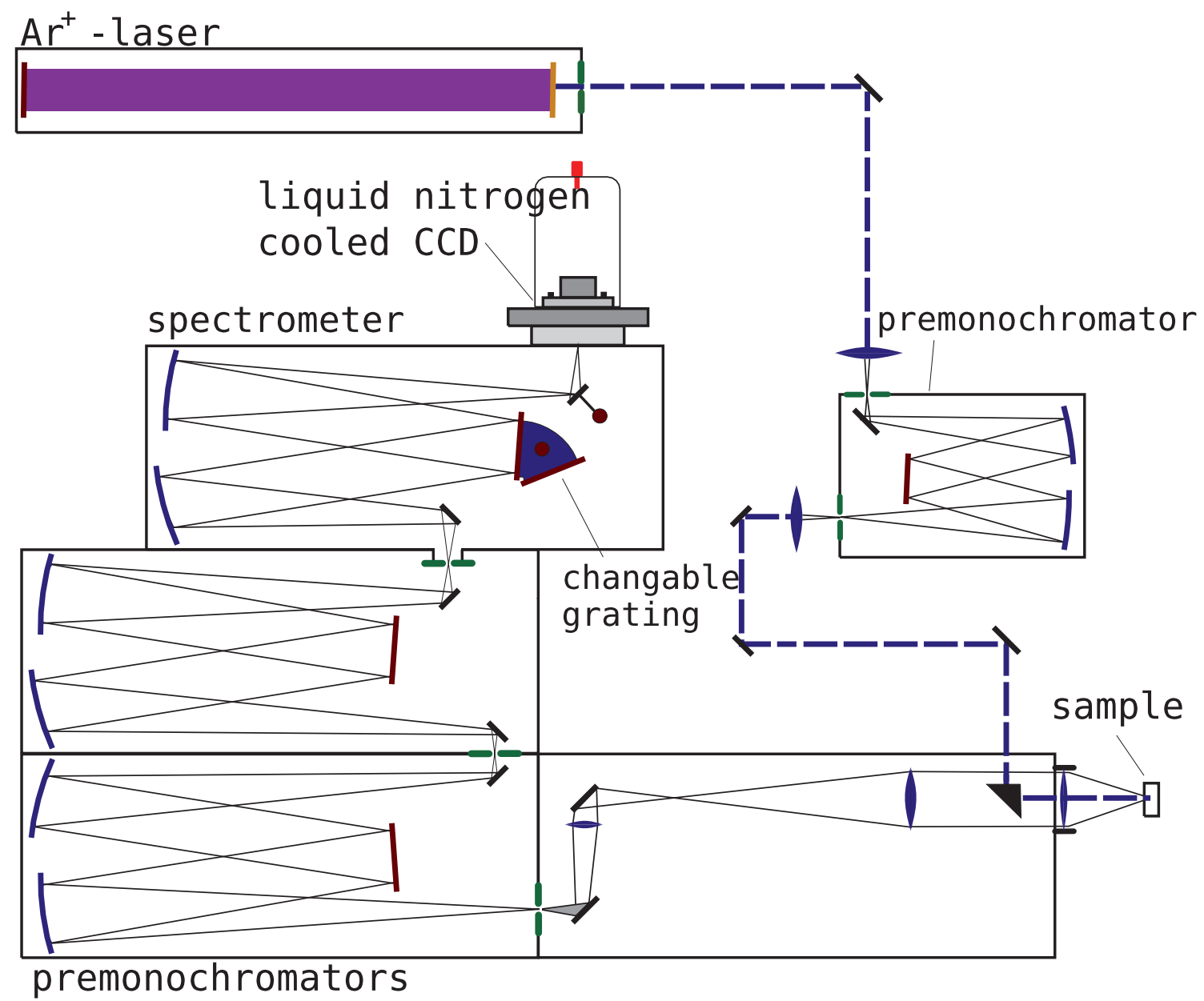

Figure 3.4: Raman spectroscopy set-up. Plasma lines are absorbed in the premonochromator, the sample area is illuminated in a confocal beam path and a Dylor-XY triple-monochromator system with a CCD camera is used to analyze the scattered light.

spectrum. The premonochromator can be tuned to the laser wavelength and cuts of possible plasma lines. It can be replaced by a dielectric band-pass filter, which works just for a single wavelength, but more efficiently and disturbs the beam profile less. A Dylor-XY triple-monochromator system is used as Raman system. The laser light enters the system and illuminates the sample in a confocal, macroscopic optical path via a prism. The spot size is approx. $1 \mathrm{~mm}^{2}$ and the laser power is kept below $10 \mathrm{~mW}$ to avoid laser heating and ensure constant sample temperatures. The sample area is large enough to e.g. host a cryostat for low temperature measurements and specific gaseous atmospheres as surrounding media. The scattered light re-enters the Raman system and two premonochromators act as band-pass for the Raman part of the scattered light. The third monochromator is the spectrometer 
Chapter 3. Methods

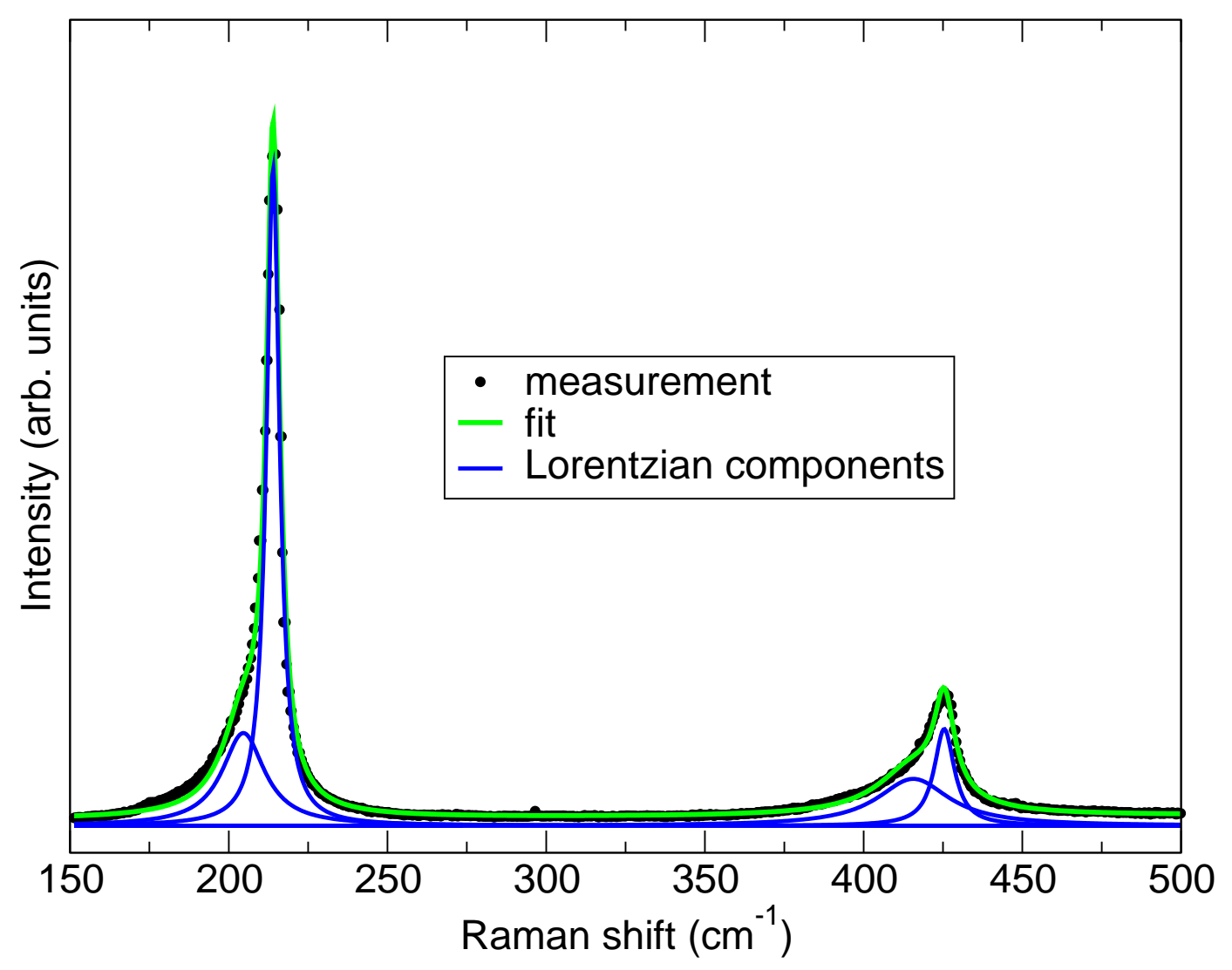

Figure 3.5: Raman spectrum of CdSe nanorods with $7 \mathrm{~nm}$ diameter and $35 \mathrm{~nm}$ length with underlying Lorentzian functions and resulting fit.

and images the Raman spectrum on a nitrogen-cooled CCD, which is used to acquire the spectra.

For the Raman measurements, the CdSe nanorods are solved in chloroform or toluene. The nanorod solution is then dripped on $5 \times 5 \mathrm{~mm}^{2}$ Si-wafers and left until the solvent has evaporated. The nanorod density on the Si surface is approx. $10^{10} \mathrm{~mm}^{-2}$. Multiple wafers can be hosted in a micro-cryostat. All Raman measurements are performed in a commercial Oxford cryostat at liquid helium temperatures.

Figure 3.5 displays a Raman spectrum in the Stokes-regime of CdSe nanorods with $7 \mathrm{~nm}$ diameter and $35 \mathrm{~nm}$ length at low temperature, acquired with this set-up.

The fundamental, first-order, Raman band is located around $200 \mathrm{~cm}^{-1}$. The band has an asymmetric shape, which is common for low-dimensional CdSe structures[68, 69] and similar materials[70]. The fundamental Raman band is assembled from two bands, each with different origin. The two contributions can each be fitted with a Lorentzian function and the sum of both describes the shape of the band very 
well. Of these two peaks, the more pronounced peak, which is mainly responsible for the position and intensity of the band can be attributed to the CdSe LO. The second feature is related to surface optical phonons (SO) and is located at the lowenergy shoulder of the LO peak and is responsible for the asymmetry of the Raman band. The frequency and intensity of the surface optical phonons is very sensitive to the surrounding of the nanorod. Gupta et al. performed Raman measurements of $\mathrm{GaP}$ nanowires, which are similar to the CdSe nanorods, in various dielectric surroundings and were able to verify the nature of the low-frequency shoulder and demonstrate the dependence on the surrounding[70]. The two contributions and their dependencies will be discussed in detail in Chapter 4 .

At larger wavenumbers, around $400 \mathrm{~cm}^{-1}$, the second-order Raman band can be observed. The relative intensity of the second-order band to the fundamental Raman band depends on the coupling strength between the phonons and excited charge carriers. This dependence will be exploited in Chapter 5, when discussion the excitonphonon coupling strength. 



\section{Chapter 4}

\section{Geometry dependence of the phonon modes in CdSe nanorods}

In Chapter 2 the CdSe nanorod growth and the size-tunability by the variation of the growth parameters were presented. The fundamental Raman band of CdSe nanorods and its two main contributions were briefly discussed in Chapter 3. In this chapter the size-tunability of the nanorods is exploited to systemmatically study confinement effects on these two contributions.

\subsection{Size-dependent Raman spectra}

To investigate the dependencies of the contributions to the fundamental Raman band on the nanorod size, nanorods of different dimensions are measured. The Raman spectrum of nanorods with $7 \mathrm{~nm}$ diameter and $35 \mathrm{~nm}$ length is displayed in Figure 4.1. As mentioned in Chapter 3, the Raman band has an asymmetric shape. It is composed of the $\mathrm{LO}$ and $\mathrm{SO}$.

In Figure 4.2 the Raman spectrum of nanorods with $8 \mathrm{~nm}$ diameter and $80 \mathrm{~nm}$ length is displayed. These nanorods only have a slightly larger diameter than the $7 \times 35 \mathrm{~nm}^{2}$ nanorods, while the aspect ratio is much larger. It is twice the aspect ratio of the $7 \times 35 \mathrm{~nm}^{2}$ nanorods. The LO frequency is slightly different between the two nanorods. It is shifted down by about one wavenumber between the $7 \times 35 \mathrm{~nm}^{2}$ and $8 \times 80 \mathrm{~nm}^{2}$ nanorods. The frequency of the SO related band changes more drastically and its frequency is increased by $5 \mathrm{~cm}^{-1}$. The sum of both changes results in a slightly different position and a quite different shape of the fundamental Raman band. 
Chapter 4. Geometry dependence of the phonon modes in CdSe nanorods

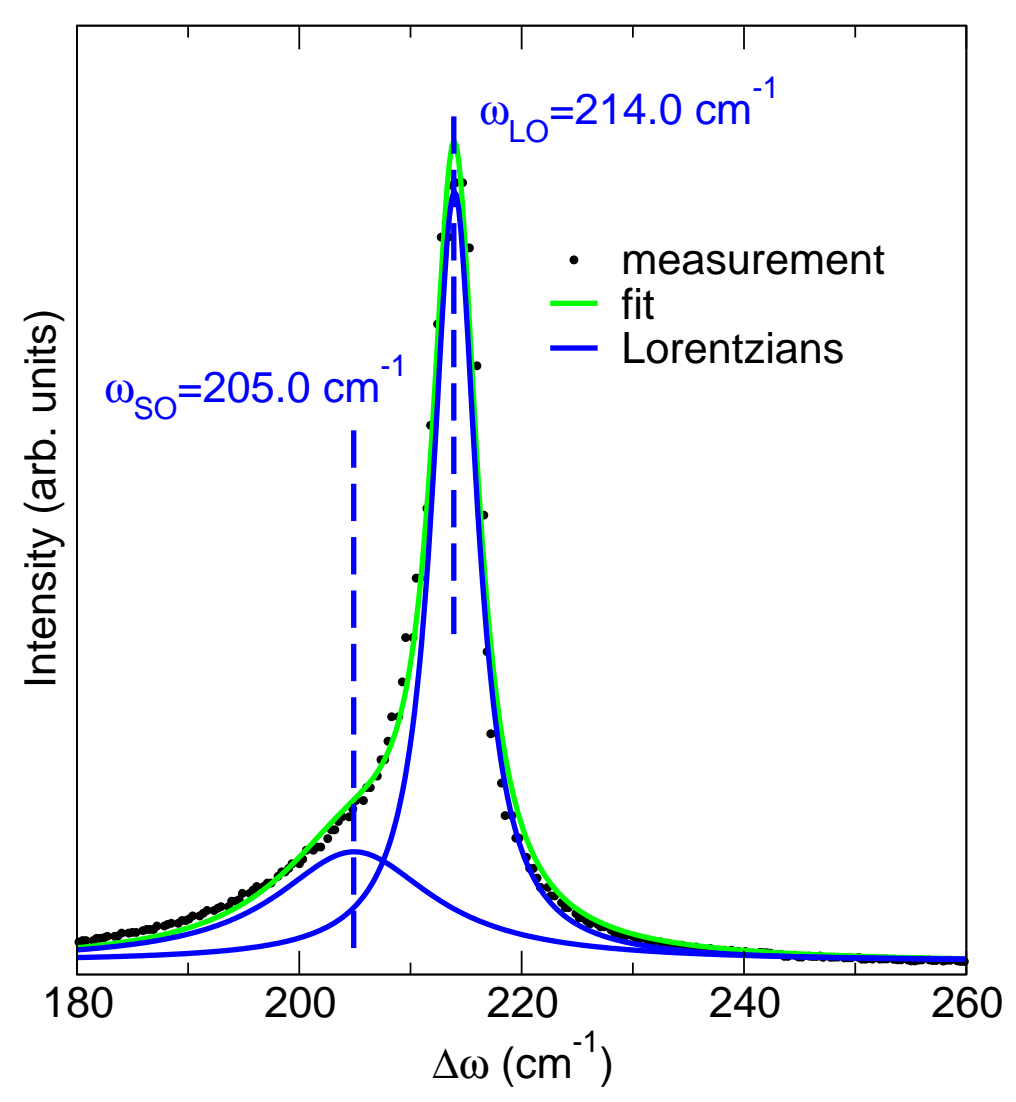

Figure 4.1: Raman spectrum of CdSe nanorods with $7 \mathrm{~nm}$ diameter and $35 \mathrm{~nm}$ length.

The Raman spectrum of thinner nanorods is displayed in Figure 4.3. It shows the Raman spectrum of nanorods with $4 \mathrm{~nm}$ diameter and $30 \mathrm{~nm}$ length. Here, strong deviations of the LO frequency from the two thicker nanorods can be observed, while the SO related peak is shifted less than in the previous example. This results in a quiet symmetric band at a different position.

The two contributions to the fundamental Raman band obviously depend on the nanorod geometry. As diameter and aspect ratio changes affect the SO and LO frequency each in different ways, the two contributions seem to have different dependencies on the nanorod geometry. Exploiting the size-tunability and measuring several nanorod samples of different sizes allows a systematic investigation. The LO and SO frequencies for five exemplary geometries are summarized in Table 4.1.

The LO frequency of two nanorod samples with the same diameter $(4 \mathrm{~nm})$, but different lengths $(25 \mathrm{~nm}$ and $30 \mathrm{~nm}$ ) is experimentally not separable, the frequency can thus be considered to be mostly independent of the nanorod length. The second 


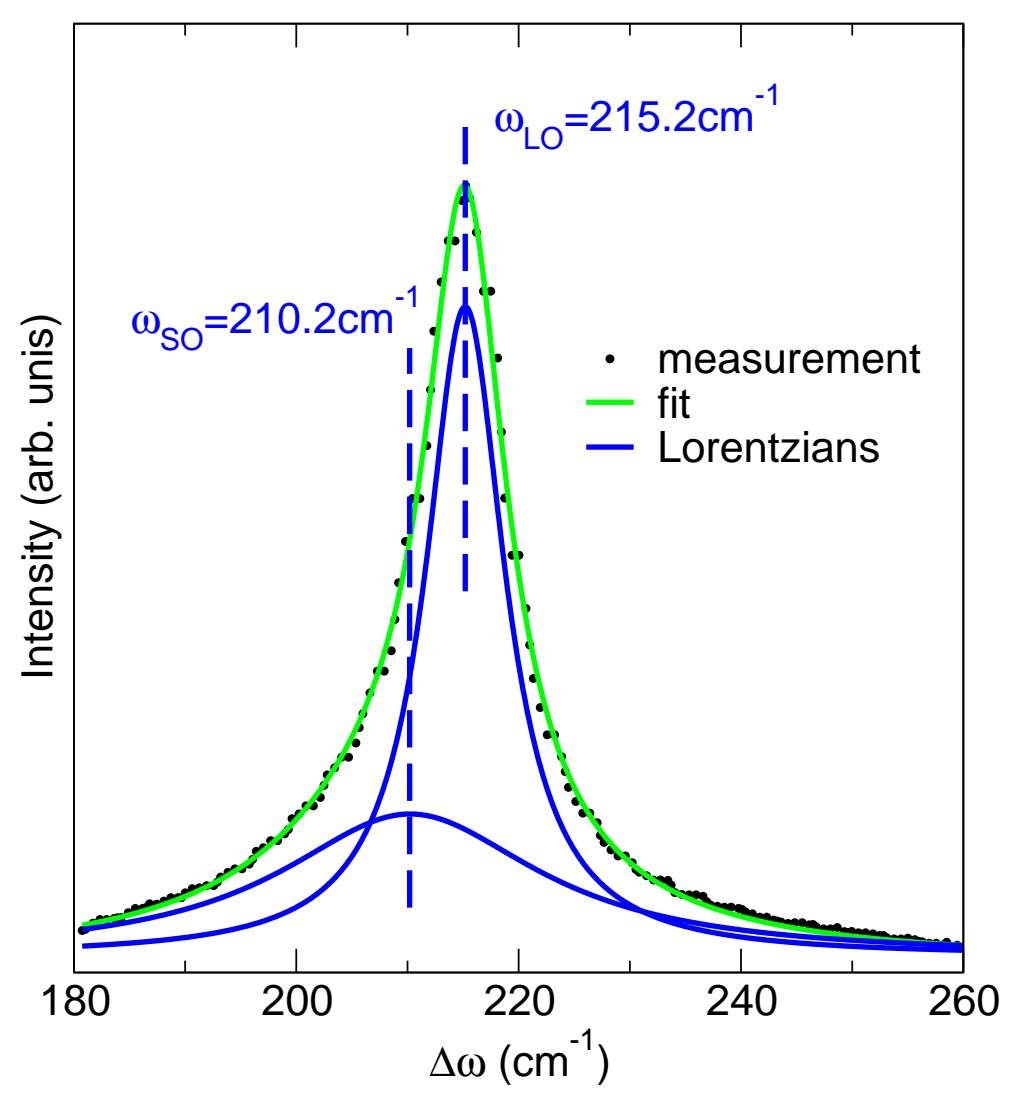

Figure 4.2: Raman spectrum of CdSe nanorods with $8 \mathrm{~nm}$ diameter and $80 \mathrm{~nm}$ length.

contribution to the fundamental Raman band, the SO, behaves quite differently. While the frequency of the LO is mostly diameter dependent, the SO frequency also depends on the nanorod length. The SO frequency is different for two nanorods of the same diameter, but with different lengths. However, a pure length dependence can be excluded. The SO frequency of nanorods with $8 \mathrm{~nm}$ diameter and $80 \mathrm{~nm}$ length differs only by $1.5 \mathrm{~cm}^{-1}$ from the SO frequency of nanorods with $4 \mathrm{~nm}$ diameter and $30 \mathrm{~nm}$ length. However, it differs by $3.7 \mathrm{~cm}^{-1}$ between nanorods with $4 \mathrm{~nm}$ diameter and $30 \mathrm{~nm}$ length and nanorods with $7 \mathrm{~nm}$ diameter and $35 \mathrm{~nm}$ length, where the absolute and relative differences in length are much smaller. Thus, the combination of the diameter and length must be relevant.

The influence of the nanorod geometry on the LO and SO frequency will be treated separately and explained within established theory for phonon confinement and surface optical phonons in the following sections. 
Chapter 4. Geometry dependence of the phonon modes in CdSe nanorods

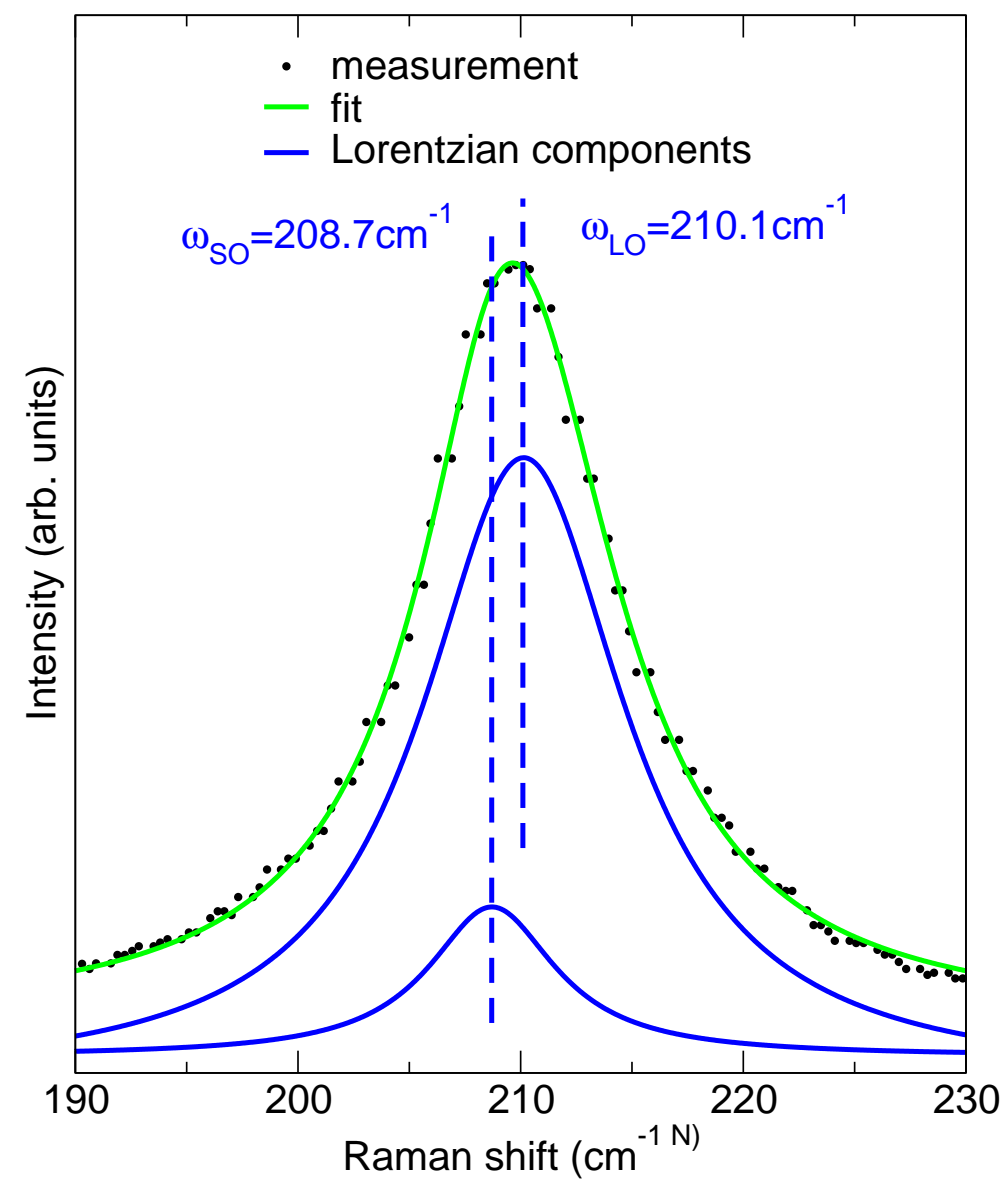

Figure 4.3: Raman spectrum of CdSe nanorods with $4 \mathrm{~nm}$ diameter and $30 \mathrm{~nm}$ length.

Table 4.1: Frequencies of the $\mathrm{LO}$ and $\mathrm{SO}, \omega_{\mathrm{LO}}$ and $\omega_{\mathrm{SO}}$, for different nanorod geometries.

\begin{tabular}{ccc} 
sample dimesions & $\omega_{\mathrm{LO}}\left(\mathrm{cm}^{-1}\right)$ & $\omega_{\mathrm{SO}}\left(\mathrm{cm}^{-1}\right)$ \\
\hline $3.5 \mathrm{~nm} \times 20 \mathrm{~nm}$ & $209.2 \pm 0.6$ & $206.4 \pm 1.2$ \\
$4 \mathrm{~nm} \times 25 \mathrm{~nm}$ & $210.0 \pm 0.6$ & $207.6 \pm 1.0$ \\
$4 \mathrm{~nm} \times 30 \mathrm{~nm}$ & $210.1 \pm 0.5$ & $208.7 \pm 0.9$ \\
$7 \mathrm{~nm} \times 35 \mathrm{~nm}$ & $214.0 \pm 0.5$ & $205.0 \pm 0.9$ \\
$8 \mathrm{~nm} \times 80 \mathrm{~nm}$ & $215.2 \pm 0.5$ & $210.2 \pm 1.0$
\end{tabular}




\subsection{Phonon confinement}

In semiconductor nanostructures like CdSe nanorods, the phonon wavefunction is restricted to the volume of the nanocrystal. This leads to the relaxation of the conservation of crystal momentum in the creation and decay of phonons in the nanocrystal. A general model for confined phonons was initially developed by Richter et al. and applied for low-dimensional silicon structures[71]. Campbell and Fauchet further generalized the model[72].

According to Richter et al. the wavefunction of a phonon with the wavevector $\mathbf{q}_{0}$ can be written as

$$
\Phi\left(\mathbf{q}_{\mathbf{0}}, \mathbf{r}\right)=u\left(\mathbf{q}_{\mathbf{0}}, \mathbf{r}\right) e^{-i \mathbf{q}_{\mathbf{0}} \cdot \mathbf{r}},
$$

where $u\left(\mathbf{q}_{\mathbf{0}}, \mathbf{r}\right)$ has the periodicity of the crystal lattice. Richter et al. treated the problem of localized phonons by the simplest possible manner and assumed the crystal to be spherical. The wavefunction is expected to be localized in the form of a Gauss-distribution. This can be treated by replacing the wavefunction $\Phi$ with a wavefunction $\Psi$ according to

$$
\begin{aligned}
\Psi\left(\mathbf{q}_{\mathbf{0}}, \mathbf{r}\right) & =W\left(\mathbf{q}_{\mathbf{0}}, \mathbf{r}\right) \Phi\left(\mathbf{q}_{\mathbf{0}}, \mathbf{r}\right) \\
& =A \cdot \exp \left(-\left(r^{2} / 2\right) /(L / 2)^{2}\right) \Phi\left(\mathbf{q}_{\mathbf{0}}\right) \\
& =\Psi^{\prime}\left(\mathbf{q}_{\mathbf{0}}, \mathbf{r}\right) \cdot u\left(\mathbf{q}_{\mathbf{0}}, \mathbf{r}\right)
\end{aligned}
$$

with $|\Psi|=A^{2} \exp \left(-r^{2} /(L / 2)^{2}\right) . W\left(\mathbf{q}_{\mathbf{0}}, \mathbf{r}\right)$ is the weighting function and $\mathrm{L}$ is the diameter of the sphere.

To calculate the effect on the Raman spectrum, $\Psi^{\prime}$ is expanded in a Fourier series

$$
\Psi^{\prime}\left(\mathbf{q}_{\mathbf{0}}, \mathbf{r}\right)=\int d^{3} q C\left(\mathbf{q}_{\mathbf{0}}, \mathbf{q}\right) e^{i \mathbf{q} \cdot \mathbf{r}},
$$

with the Fourier coefficients determined by

$$
C\left(\mathbf{q}_{\mathbf{0}}, \mathbf{q}\right)=\frac{1}{(2 \pi)^{3}} \int d^{3} r \Psi^{\prime}\left(\mathbf{q}_{\mathbf{0}}, \mathbf{r}\right) e^{-i \mathbf{q} \cdot \mathbf{r}} .
$$

The phonon wavefunction is a superposition of eigenfunctions with wavevector $\mathbf{q}$, centered at $\mathbf{q}_{\mathbf{0}}$. When scale factors are neglected and $\mathbf{q}_{\mathbf{0}}=0$ is assumed for onephonon processes, the Fourier coefficients for a spherical crystal become

$$
\mid C\left(0,\left.q\right|^{2} \cong e^{-q^{2} L^{2} / 4} .\right.
$$

The first order Raman spectrum can then be calculated from

$$
I(\omega)=\int \frac{d^{3} q|C(0, \mathbf{q})|^{2}}{(\omega-\omega(\mathbf{q}))^{2}+\left(\Gamma_{0} / 2\right)^{2}},
$$


Chapter 4. Geometry dependence of the phonon modes in CdSe nanorods

where $\omega(\mathbf{q})$ is the phonon dispersion and $\Gamma_{0}$ is the natural linewidth.

Campbell and Fauchet argued that there is no real physical reason for $W\left(\mathbf{q}_{\mathbf{0}}, \mathbf{r}\right)$ to be a Gaussian, $\exp \left(-r^{2} /(L / 2)^{2}\right)$, with a phonon amplitude of $1 / e$ at the boundary of the nanocrystal, as chosen by Richter et al. This pure Gaussian ansatz yields satisfying results for certain material systems like the Si spheres discussed by Richter et al., but fails for other structures like the GaAs spheres that Campbell and Fauchet presented [72]. To reflect the specialties of the system under investigation one can modify the form of the confinement function along with the phonon wavefunction amplitude at the crystal boundary. Furthermore one can consider the effect of the nanocrystals' shape and include confinement in multiple dimensions. For a column and a Gaussian confinement this results in the Fourier coefficients

$$
\left|C\left(0, q_{1}, q_{2}\right)\right|^{2} \cong e^{-\alpha q_{1}^{2} L_{1}^{2} / 16 \pi^{2}} e^{-\alpha q_{2}^{2} L_{2}^{2} / 16 \pi^{2}} \times\left|1-\operatorname{erf}\left(\frac{i q_{2} L_{2}^{2}}{\sqrt{32 \pi}}\right)\right|^{2},
$$

where $L_{2}$ is the length of the cylindric column, $L_{1}$ the diameter. The variable confinement parameter $\alpha$ is introduced to allow a "tuning" of the actual strength of the confinement in order to reflect the available experimental data[72].

To numerically calculate the Raman spectrum of confined phonons one integrates equation 4.6 with a fixed frequency $\omega$ over the whole Brillouin zone and then stepwise varies the frequency.

For the CdSe nanorods with their large aspect ratios, the length can be considered to be infinite and the $L_{2}$-terms in equation 4.7 can be neglected. This is justified by the length-independence of the LO frequency in the experimental data. The integration over the Brillouin zone can be performed by a numerical integration over the available directions of the phonon dispersion and an averaging. The phonon dispersion calculated by Mohr et al., presented in Chapter 3, is used for the integration. To treat phonon confinement in nanorods with the model presented above, one has to estimate the confinement parameter $\alpha$ in equation 4.7 for the given geometry. It can be estimated by adjusting a calculated spectrum with a fixed diameter to an available experimental Raman spectrum of nanorods with the same diameter. First, a Raman spectrum is calculated for a fixed diameter, e.g. $d=4 \mathrm{~nm}$ without an initial modification of the confinement parameter, that means $\alpha=1$. After the calculation, a Lorentzian function can be fitted to the calculated spectrum. An exemplary outcome for nanorods with a diameter of $4 \mathrm{~nm}$ is displayed in Figure 4.4.

The position and width of the fitted Lorentzian function can then be compared to the position and width of the LO band in the Raman spectrum of CdSe nanorods with the same diameter. The confinement parameter is then varied in an iterative process until the agreement between the calculated and the measured LO Raman band is maximized. For the CdSe nanorods, only small deviations from the Gaussian form 


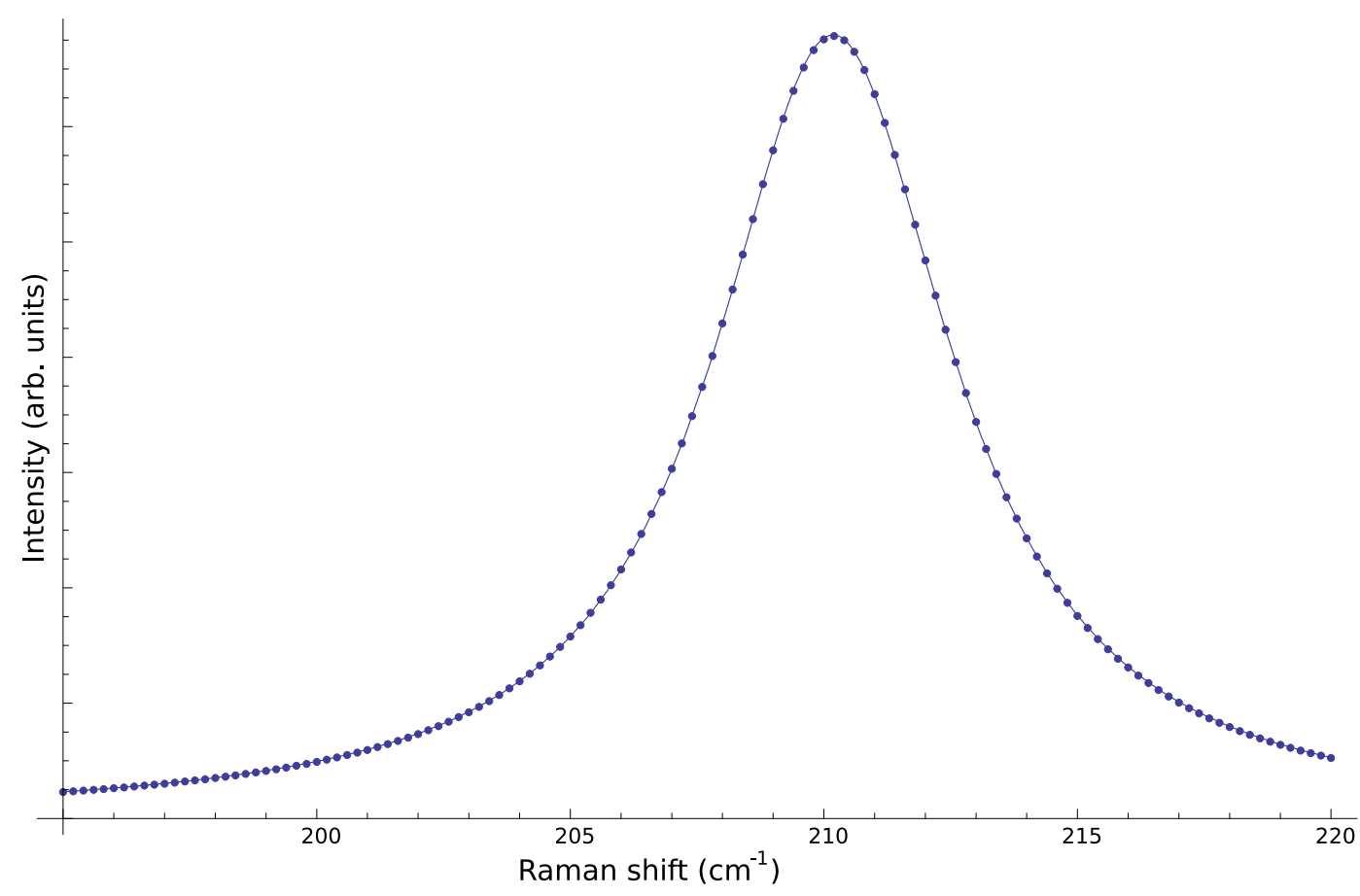

Figure 4.4: Calculated Raman spectrum for a CdSe nanorod with $4 \mathrm{~nm}$ diameter without an adjusted confinement parameter (dots) and fitted Lorentzian function (solid line) .

of confinement proposed by Campbell and Fauchet are obtained. The confinement parameter $\alpha=1.1$ yields a good agreement of the calculated LO Raman band with the experimental data.

By using this as fixed confinement parameter and varying the diameter, the influence of the confinement on the LO frequencies can be investigated. The LO frequencies for different diameters can be calculated. Figure 4.5 displays the frequencies obtained from the confinement model.

The LO frequencies from the confinement model decrease for decreasing nanorod diameter. The shape of the phonon dispersion curve close to the $\Gamma$-point is responsible for this. A cutout of the applied disperion ist displayed in Figure 4.6.

The stronger the confinement the more phonons far from the $\Gamma$-point contribute to the Raman scattering. The CdSe bulk phonon dispersion shows an overbending near the $\Gamma$-point, which can be found in other hexagonal lattices as well[73, 74]. This leads to the observed downshift behavior.

Included in Figure 4.5 are the LO frequencies obtained from the Raman measurements. The model for confined phonons describes the influence of the decreasing diameter on the frequency of the longitudinal phonon correctly. A saturation for 
Chapter 4. Geometry dependence of the phonon modes in CdSe nanorods

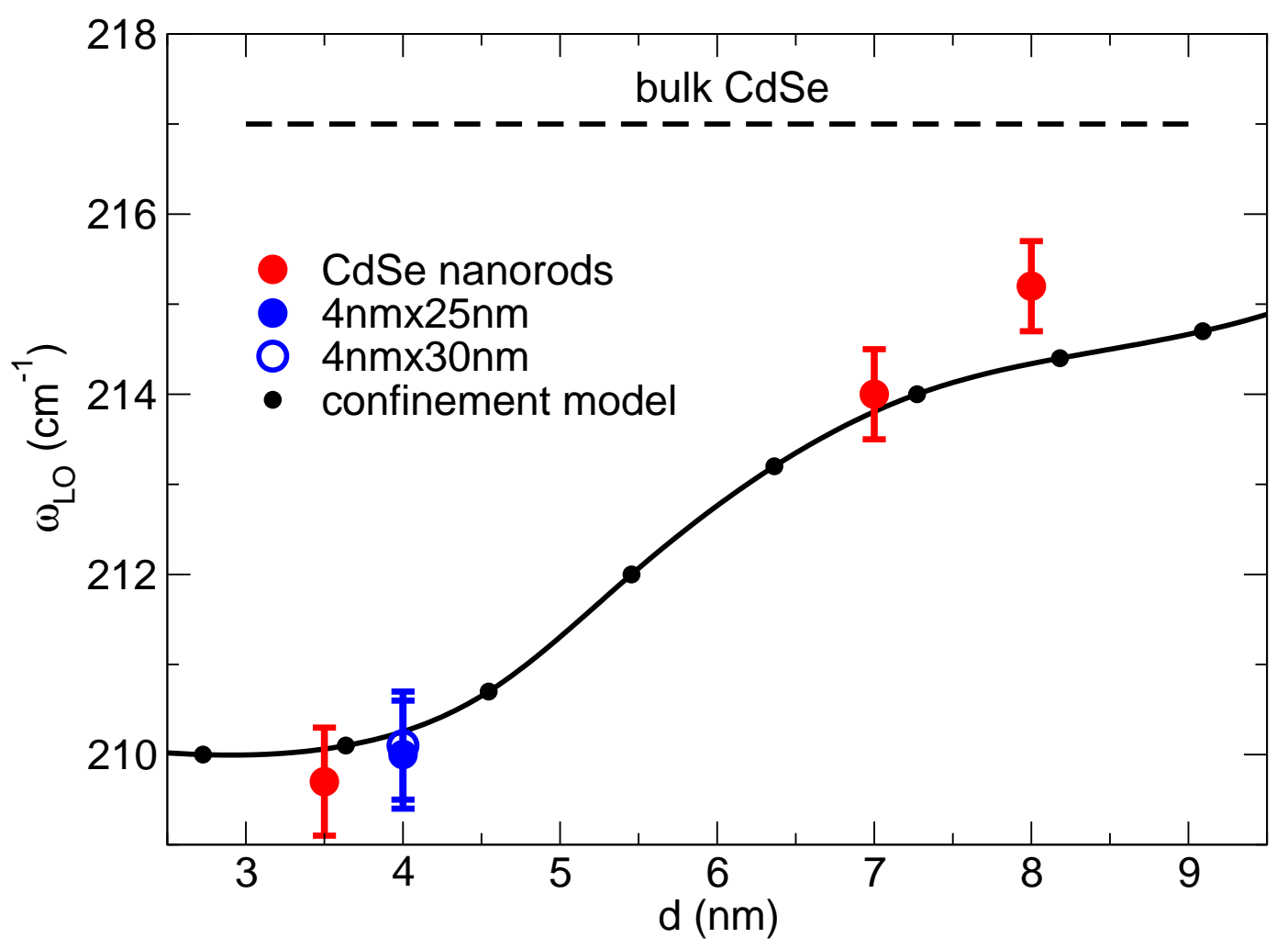

Figure 4.5: Measured LO frequency and calculated frequencies versus nanorod diameter. The solid line is a guide to the eye.

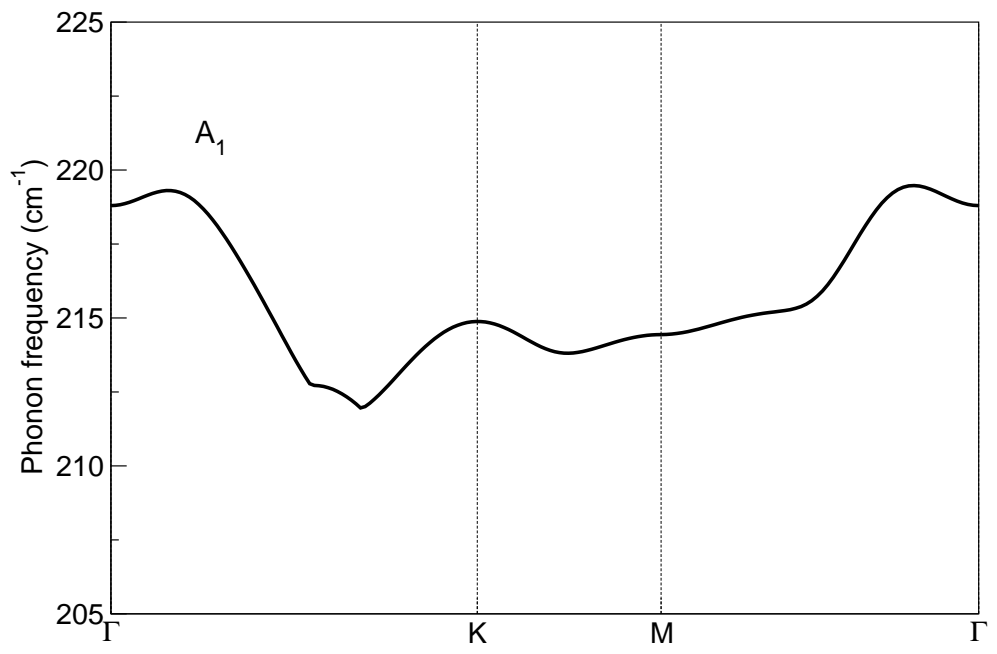

Figure 4.6: Ab-initio calculated longitudinal optical phonon dispersion. 
very small diameters is found in the model and agrees with the experimental data. For diameters above $7 \mathrm{~nm}$, the model indicates a stronger saturation of the frequency shift than found in the experimental data. In summary, the phonon confinement model accounts for the observed variations in the frequency of the LO of CdSe nanorods. Other effects like deviations from the cylindrical shape or surface effects are not considered by the model and could e.g. lead to a weaker saturation of the confinement influence. The quantitative agreement between the calculations and the measurements indicates, however, that the relaxation of the wave vector selection rule is most relevant for the LO frequency in CdSe nanorods. The good agreement between theory and experiment furthermore shows, that longitudinal optical phonons are, in average, not strongly influenced by the diameter fluctuations mentioned in Chapter 2. 
Chapter 4. Geometry dependence of the phonon modes in CdSe nanorods

\subsection{Surface optical phonons}

The size-dependence of the LO frequency discussed above can be explained in terms of phonon confinement. The different size-dependence of the SO frequency can be understood when calculating the SO frequencies with a quantum mechanical continuum approach.

A theoretical treatment of $\mathrm{SO}$ modes in nanocrystals was established by Comas et al. [69, 75]. They investigate polar optical vibrations in small-size crystals by applying a dielectric continuum approach, based on established theory[76]. In this approach, both bulk-like oscillations and surface modes are included in the study of the vibrations of finite crystals. The quantum version of such oscillatory modes are the longitudinal optical phonons, transverse optical phonons and the corresponding surface optical phonons.

The Born-Huang equation of motion applies

$$
\ddot{\omega}=-\omega_{T}^{2} \omega+\sqrt{\frac{\left(\epsilon_{0}-\epsilon_{\infty}\right)}{4 \pi} \omega_{T}^{2}} \mathbf{E},
$$

with the electric field $\mathbf{E}$, the transverse limit bulk frequency $\omega_{T}$ and the static and high frequency dielectric constants of the poloar material $\epsilon_{0}$ and $\epsilon_{\infty}$.

For the relative displacement $u$ between a pair of ions of reduced mass $\mu$ in a polar crystal of concentration $N$ (the reciprocal of the unit-cell volume) the polarization $\mathbf{P}$ is given by

$$
\mathbf{P}=\sqrt{\frac{\left(\epsilon_{0}-\epsilon_{\infty}\right)}{4 \pi} \omega_{T}^{2}} \omega+\frac{\epsilon_{\infty}-1}{4 \pi} \mathbf{E},
$$

with $\omega=\sqrt{N \mu} u$.

The Lyddane-Sachs-Teller relation $\omega_{L}^{2} / \omega_{T}^{2}=\epsilon_{0} / \epsilon_{\infty}$ applies[69].

The electric field has to satisfy the quasi-static Maxwell equations. This requires the induction field $\mathbf{D}=\epsilon(\omega) \mathbf{E}=\mathbf{E}+4 \pi \mathbf{P}$ to fulfill the Gauss equation $\nabla \cdot \mathbf{D}=0$. The relation $E-\nabla \varphi$ then leads to the main equation of the dielectric approach:

$$
\epsilon(\omega) \nabla^{2} \varphi=0
$$

The harmonic time dependence is implicit, the frequency-dependent dielectric function is thus

$$
\epsilon(\omega)=\epsilon_{\infty} \frac{\left(\omega^{2}-\omega_{L}^{2}\right)}{\left(\omega^{2}-\omega_{T}^{2}\right)} .
$$

The relevant potentials for the SO all satisfy the Laplace equation $\nabla^{2} \varphi=0$. The boundary condition then requires the normal component of $\mathbf{D}$ to be continuous at the interface between the nanocrystal and its surrounding medium. The geometry of the nanorod can be described in spheroidal coordinates. The equation

$$
\xi=\xi_{0}=\text { constant }
$$


describes an ellipsoid of revolution where the z-axis (revolution axis) is taken along the major ellipsoid axis. For such a geometry in spheroidal coordinates the Laplace equation is separable and leads to the eigenfrequencies $\omega_{l m}$ for the SO:

$$
\frac{\omega_{l m}^{2}}{\omega_{T}^{2}}=\frac{\epsilon_{0}-\epsilon_{D} f_{l m}^{P}\left(\xi_{0}\right)}{\epsilon_{\infty}-\epsilon_{D} f_{l m}^{P}\left(\xi_{0}\right)}
$$

$\xi_{D}$ is the dielectric constant of the surrounding material. The universal parameters $f_{l m}^{P}\left(\xi_{0}\right)$ are defined as

$$
f_{l m}^{P}\left(\xi_{0}\right)=\frac{\epsilon(\omega)}{\epsilon_{D}}=\left(\left.\frac{d}{d \xi} \ln Q_{l}^{m}\right|_{\xi_{0}}\right)\left(\left.\frac{d}{d \xi} \ln R_{l}^{m}\right|_{\xi_{0}}\right)^{-1}
$$

The functions $R_{L}^{m}(\xi)$ and $Q_{L}^{m}(\xi)$ are given in terms of the hypergeometric function as

$$
R_{l}^{m}(\xi)=\frac{(2 l) !\left(\xi^{2}-1\right)^{m / 2} \xi^{l-m}}{2^{l} l !(l-m) !} F\left[\frac{m-l}{2}, \frac{m-l+1}{2}, \frac{1}{2}-l, \frac{1}{\xi^{2}}\right],
$$

and

$$
Q_{l}^{m}(\xi)=\frac{2^{m}(l-m) ! \Gamma(1 / 2)\left(\xi^{2}-1\right)^{m / 2}}{\Gamma(l+3 / 2)(2 \xi))^{l+m+1}} F\left[\frac{l+m+1}{2}, \frac{l+m+2}{2}, l+\frac{3}{2}, \frac{1}{\xi^{2}}\right] .
$$

The universal parameters $f_{l m}^{P}\left(\xi_{0}\right)$ are independent of the nature of the constituent materials. They do, however, depend on the nanocrystals dimensions via $\xi_{0}$. Within the framework of the prolate spheroidal coordinates, the ratio between the semiaxes can be identified as the aspect ratio of a nanorod, which is then given by $r=$ $\xi_{0} / \sqrt{\xi_{0}^{2}-1}$. The definition of the prolate spheroidal coordinates allows the aspect ratio $r$ to be varied in an interval between 2 and 10 .

The frequency of the SO then depends on the nanorod material, the hosting material, which are CdSe and air, the nanorods' aspect ratio and the choice of the quantum numbers $l, m$. From the possible set of quantum numbers, only a few are relevant for Raman measurements. In Raman backscattering experiments, some of the SO are not visible because their transition amplitudes are zero from the Raman selection rules. Only SO with $m=0$ and $l=$ even are allowed. The SO related to the quantum numbers $(l=2, m=0)$ is expected to be most prominent in the Raman spectra. Figure 4.7 displays the related SO frequency versus the aspect ratio. The required dielectric constants and the transverse optical phonon frequency are from ref. [28].

The SO frequency increases for increasing aspect ratios and shows a saturation for aspect ratios above 10 .

The measured frequencies of the SO also have a dependence on the nanorods' aspect 
Chapter 4. Geometry dependence of the phonon modes in CdSe nanorods

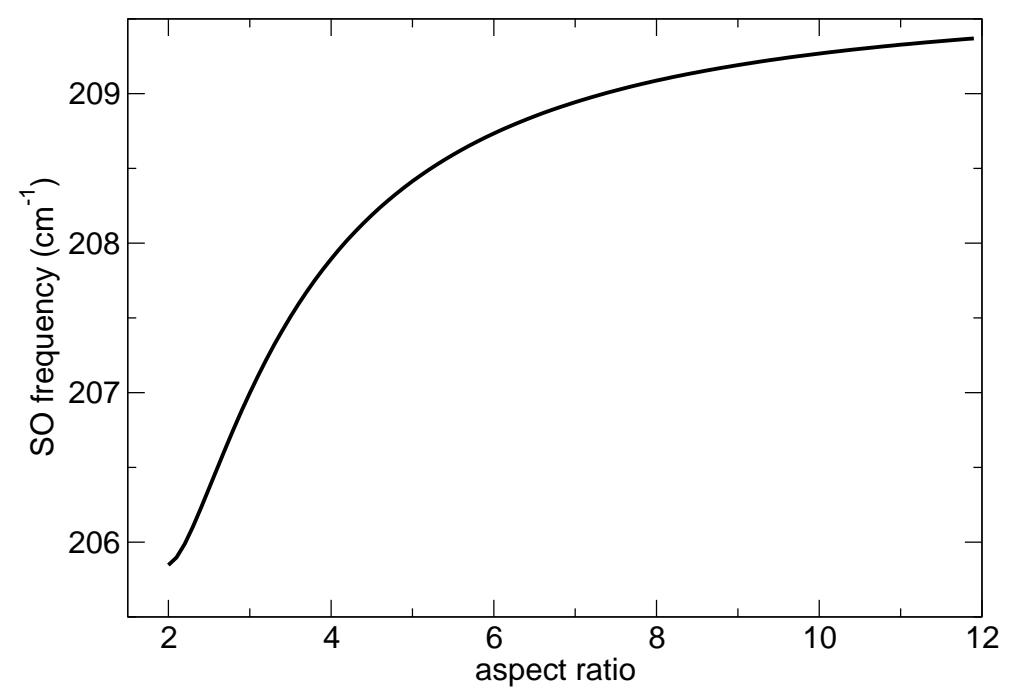

Figure 4.7: SO frequency versus nanorod aspect ratio for the quantum numbers $(l=2, m=0)$.

ratio. In Figure 4.8 the measured frequencies of the $\mathrm{SO}, \omega_{\mathrm{SO}}$ for various aspect ratios are displayed. Included in the figure is the calculated course for the quantum numbers $(l=2, m=0)$. As in the model, the SO frequency decreases for decreasing aspect ratios and shows a saturating behavior for large aspect ratios.

The shape of the calculated SO frequency versus aspect ratio dependence is similar to the course of the measured values. However, it only gives the general trend of the shift with changing aspect ratio. The strength of the shift for a changing aspect ratio seems to be underestimated by the model. Other effects that strengthen the effect must be relevant. Possible explanations for this could be contributions from other $(l, m)$-combinations that have a steeper slope. Furthermore, deviations from the perfect axially symmetrical rod-shape could also result in changed dependencies. This can, however, not be treated within the model as it has strict conditions for the prolate spheroidal coordinates. A third explanation could be organic surfactants remaining around the nanorods after the synthesis. This could result in an effective dielectric constant of the nanorod surrounding different from 1 . The influence of this can be investigated by varying the dielectric constant of the surrounding. Trioctylphosphine oxide, that is often used to saturate the nanorod surfaces, for example has a dielectric constant of $\epsilon_{D}=2.1[77]$. The SO frequency versus aspect ratio dependence for dielectric constants of the surrounding is displayed Figure 4.9.

The increase of the dielectric constant of the surrounding medium results not only in different frequencies for a fixed geometry, but also in a different aspect-ratio- 


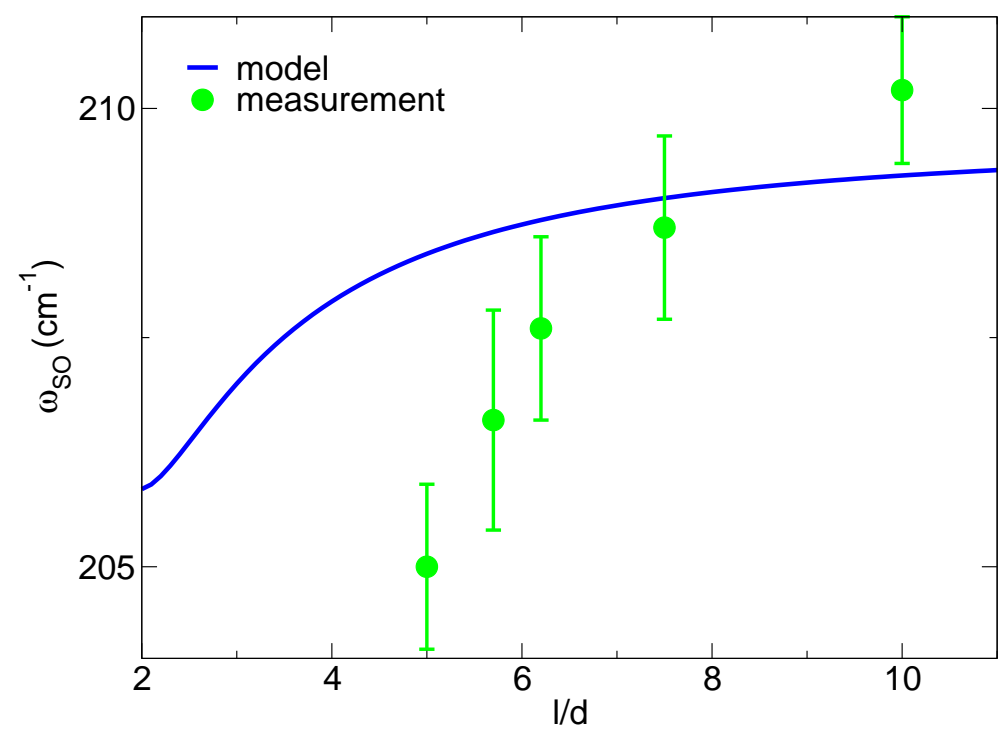

Figure 4.8: Measured SO frequency versus nanorod aspect ratio and model for the quantum numbers $(l=2, m=0)$.

dependence for a static surrounding.

In this chapter, the geometry-dependence of the LO and SO frequencies were investigated. The LO frequency changes due to phonon confinement and is purely diameter dependent. The SO frequency depends on the nanorods' aspect-ratio. The theoretical model, however, underestimates the influence of the nanorods' shape on the SO frequency.

In the next chapters the size tunability of the nanorods is further utilized to investigate the geometry-dependence of other effects that are accessible by Raman spectroscopy. 
$\epsilon_{D}$

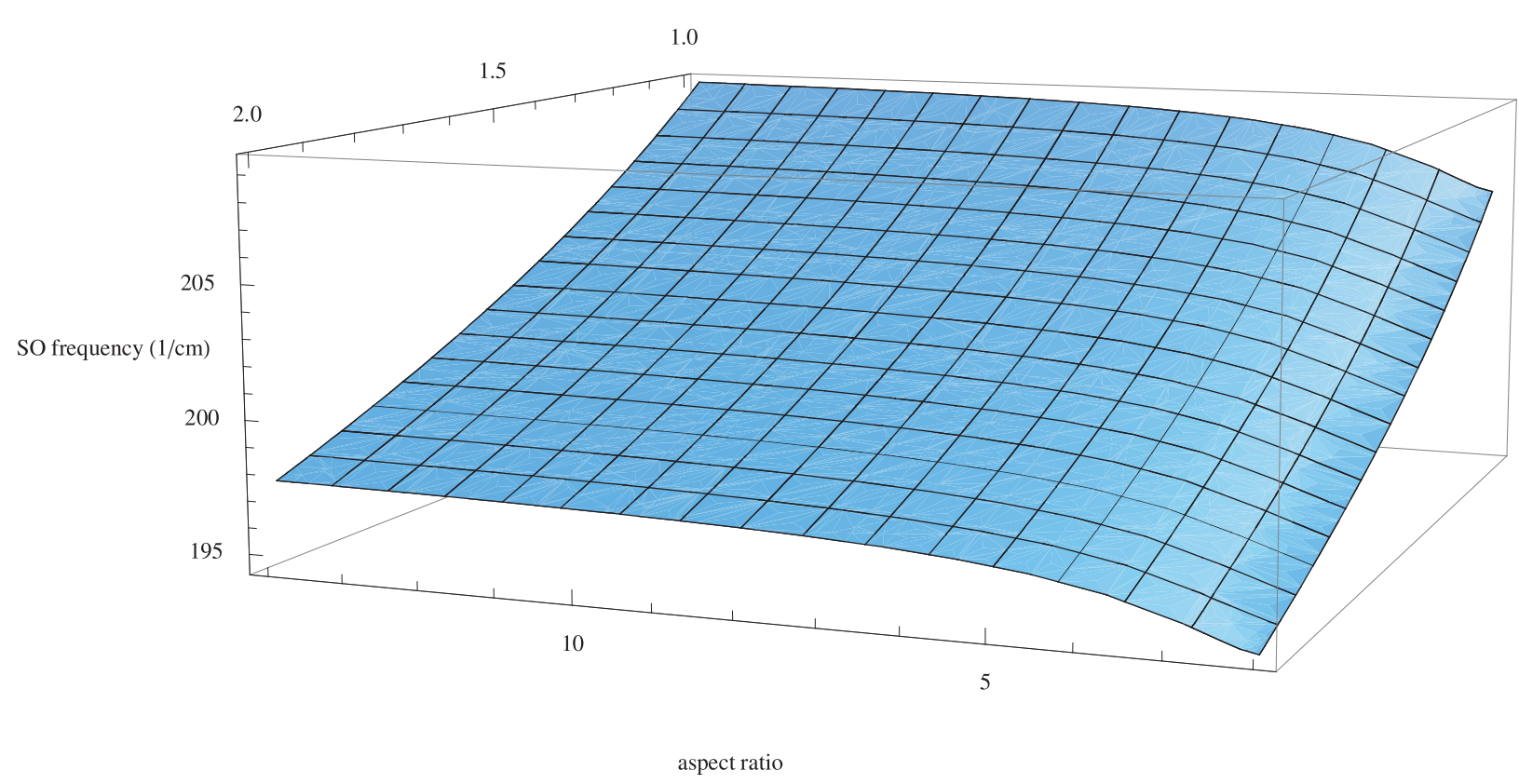

Figure 4.9: Surface optical phonon frequencies versus nanorod aspect ratio for different dieelectric constants of the surrounding medium. 


\section{Chapter 5}

\section{Exciton-LO-phonon coupling in CdSe nanorods}

In polar materials like CdSe, the optical phonons couple to electronic excitations. The coupling between vibrational and electronic excitations has an influence on many optical and electrical properties, like the transport and energy relaxation rate of excited carriers. The coupling strength can be obtained from the Raman spectra. In this chapter, the Huang-Rhys parameter, which is a quantity for the excitonphonon coupling strength, of CdSe nanorods is estimated and compared to bulk CdSe. Then, the size-tunability of the nanorods is used to investigate the influence of the nanorod geometry on the coupling strength.

\subsection{The Huang-Rhys parameter}

In polar materials the dominant coupling between electronic charge and crystal vibrations is the Fröhlich interaction between the field induced by the vibrational motion and the electronic charge density. According to Fröhlich, the forces on the ions depend exclusively on the macroscopic electric field and the relative ion displacements[78]. This model leads to pure transverse and longitudinal modes and is most valid for optical waves in which the positive and negative ions vibrate out of phase. For optical phonons in the polar material CdSe this is a suitable description. Huang and Rhys first treated an equivalent problem within a general discussion of light absorption and non-radiative transitions in F-centres[79]. There, the coupling between electronic excitations and crystal vibrations is treated on the basis of the Franck-Condon principle. The crystal is considered as a dielectric continuum and the electronic excitation as a static charge distribution. The state of the system 
Chapter 5. Exciton-LO-phonon coupling in CdSe nanorods

is specified by two quantum numbers: $\mu$ for the electronic excitation and $n$ for the vibrational states of the crystal lattice. The energy of a state is $E_{\mu n}$. In this notation, the Franck-Condon principle is written as

$$
\langle i|\mathbf{M}| f\rangle \rightarrow\left\langle\mu^{\prime}|e \mathbf{X}| \mu^{\prime \prime}\right\rangle \int \mathbf{X}_{\mu^{\prime} n^{\prime}}^{*}(\mathbf{X}) \mathbf{X}_{\mu^{\prime \prime} n^{\prime \prime}}(\mathbf{X}) d \mathbf{X} .
$$

$\left\langle\mu^{\prime}|e \mathbf{X}| \mu^{\prime \prime}\right\rangle$ is the matrix element of the electric moment of the electronic excitation between the two electronic states $\mu^{\prime}$ and $\mu^{\prime \prime}$. $\mathbf{X}$ are general coordinates, specifying the lattice configurations and $\mathbf{X}_{\mu^{\prime} n^{\prime}}$ and $\mathbf{X}_{\mu^{\prime \prime} n^{\prime \prime}}$ are the vibrational wave functions of the lattice for the electronic excitation in each state.

The lattice configuration is specified by the displacement vector $\mathbf{u}(\mathbf{r})$ of the positive ions relative to the negative ions for all positions $\mathbf{r}$ in the crystal. The total potential energy relative to the position $\mathbf{u}(\mathbf{r})=0$ in the presence of an external electric field $\mathcal{E}_{\mu^{\prime}}(\mathbf{r})$ is given by

$$
\frac{\bar{M}}{2} \int\left\{\dot{\mathbf{u}}_{l}^{2}+\omega_{l}^{2} \mathbf{u}_{l}^{2}+\dot{\mathbf{u}}_{t}^{2}+\omega_{0}^{2} \mathbf{u}_{t}^{2}\right\} \frac{d \nu}{\nu_{a}}-\omega_{l}\left[\frac{\bar{M}}{4 \pi \nu_{a}}\left(\frac{1}{\epsilon_{\infty}} \frac{1}{\epsilon_{0}}\right)\right]^{1 / 2} \int \mathbf{u}_{l} \mathcal{E}_{\mu^{\prime}} d \nu .
$$

$\mathbf{u}_{l}(\mathbf{r})$ and $\mathbf{u}_{r}(\mathbf{r})$ are the irrotational and solenoidal parts of $\mathbf{u}(\mathbf{r}), \epsilon_{0}$ and $\epsilon_{\infty}$ are the static and high frequency dielectric constants, $\bar{M}$ is the reduced mass of the ions, $\nu_{a}$ is the volume of the unit cell and $\omega_{l}=\left(\epsilon_{0} / \epsilon_{\infty}\right)^{1 / 2} \omega_{0}$. The solenoidal part of $\mathbf{u}(\mathbf{r})$ gives raise to transverse waves and does not couple to the electronic excitations and can thus be neglected in the discussion. Huang and Rhys discuss the problem in a finite volume satisfying the Born-von Karman boundary conditions. The volume integrations in equation 5.2 are restricted to a volume containing $L \times L \times L=N$ unit cells. Due to the irrotational character, $\mathbf{u}_{l}(\mathbf{r})$ can be resolved into longitudinal waves. One can then write

$$
\sqrt{N} \mathbf{u}_{l}(\mathbf{r})=\left(\frac{1}{\bar{M}}\right)^{1 / 2} \sum_{\mathbf{y}} Q(\mathbf{y})(\mathbf{y} /|\mathbf{y}|) \exp [2 \pi i \mathbf{y r}],
$$

with

$$
Q(\mathbf{y})=\frac{1}{\sqrt{2}}\left(q_{y 1}+i q_{y 2}\right)=-Q^{*}(-\mathbf{y}) .
$$

The Born-von Karman boundary conditions restrict the values of $\mathbf{y}$ by

$$
\mathbf{y}=L^{-1}\left(h^{1} \mathbf{b}_{1}+h^{2} \mathbf{b}_{2}+h^{3} \mathbf{b}_{3}\right),
$$

with $\left|h^{i}\right|=$ integer $<\frac{1}{2} L$ and $\mathbf{b}_{i}$ as the reciprocal basis vectors of the lattice. When performing the volume integration of equation 5.2 one obtains

$$
H=\frac{1}{2} \sum_{\lambda=1,2} \sum_{\mathbf{y}}\left(\dot{q}_{\mathbf{y} \lambda}^{2}+\omega_{l}^{2} q_{\mathbf{y} \lambda}^{2}\right)-\frac{1}{\sqrt{N}} \sum_{\mathbf{y} \lambda} A_{\mathbf{y} \lambda}^{\prime} q_{\mathbf{y} \lambda}
$$


where

$$
\begin{aligned}
& A_{y 1}^{\prime}=\omega_{l}\left[\frac{1}{2 \pi \nu_{a}}\left(\frac{1}{\epsilon_{\infty}}-\frac{1}{\epsilon_{0}}\right)\right]^{1 / 2} \int(\mathbf{y} /|\mathbf{y}|) \mathcal{E}_{\mu^{\prime}}(\mathbf{r}) \cos 2 \pi \mathbf{y} \cdot \mathbf{r} d \nu \\
& A_{y 2}^{\prime}=-\omega_{l}\left[\frac{1}{2 \pi \nu_{a}}\left(\frac{1}{\epsilon_{\infty}}-\frac{1}{\epsilon_{0}}\right)\right]^{1 / 2} \int(\mathbf{y} /|\mathbf{y}|) \mathcal{E}_{\mu^{\prime}}(\mathbf{r}) \sin 2 \pi \mathbf{y} \cdot \mathbf{r} d \nu
\end{aligned}
$$

When performing the summation over $\mathbf{y}$ the combination of the terms arising from $Q(\mathbf{y})$ and $Q(-\mathbf{y})$ results in only one pair of $\mathbf{y},-\mathbf{y}$ being counted. The sum over $\mathbf{y} \lambda$ can then be replaced by a single index $j$ and the Hamiltonian can be written as

$$
H=\frac{1}{2} \sum_{j}\left(\dot{q}_{j}^{2}+\omega_{l}^{2} q_{j}^{2}\right)-\frac{1}{\sqrt{N}} \sum_{j} A_{j}^{\prime} q_{j} .
$$

In the absence of an electronic excitation, the linear term in equation 5.8 drops out. The Hamiltonian then describes a system of independent oscillators of the same frequency. $q_{j}$ are then lattice coordinates and the corresponding modes are longitudinal waves. Huang and Rhys introduce the following coordinates for the presence of an electronic excitation in the state $\mu^{\prime}$ :

$$
q_{j}^{\prime}=q_{j}-\frac{1}{\sqrt{N}} \frac{A_{j}^{\prime}}{\omega_{l}^{2}} .
$$

This leads to

$$
H=\frac{1}{2} \sum_{j}\left(\dot{q}_{j}^{\prime 2}+\omega_{l}^{2} q_{j}^{\prime 2}\right)-\frac{1}{2 N} \sum \frac{A_{j}^{\prime 2}}{\omega_{l}^{2}} .
$$

The Hamiltonian is composed of the lattice vibration energy and the energy of the state $\mu^{\prime}$ of the electronic excitation. $\dot{q}_{j}^{\prime}$ is the canonical conjugate of $q_{j}^{\prime}$ and the vibrational Hamiltonian of the lattice is

$$
H_{\mathrm{vib}}=\frac{1}{2} \sum_{j}\left(-\hbar^{2} \frac{\partial^{2}}{\partial q_{j}^{\prime 2}}+\omega_{l}^{2} q_{j}^{\prime 2}\right)
$$

The wavefunctions are the products of oscillator wave functions

$$
\prod_{j} X_{n_{j}^{\prime}}\left(q_{j}^{\prime}\right)
$$

with the quantum number $n_{j}^{\prime}$ for the oscillator $j$. The overlap integrals between the vibrational wave functions $\int \mathbf{X}_{n_{j}^{\prime}} \mathbf{X}_{n_{j}^{\prime \prime}}^{(s)} d q_{j}$ (the superscript is the order of derivative) vanishes if $\left|n_{j}^{\prime}-n_{j}^{\prime \prime}\right|>s$. Transitions were the change of the quantum numbers is 
Chapter 5. Exciton-LO-phonon coupling in CdSe nanorods

greater than one can be ignored[79]. The overlap integrals are then

$$
\begin{aligned}
& \int \mathbf{X}_{n_{j}^{\prime}}\left(q_{j}^{\prime}\right) \mathbf{X}_{n_{j}^{\prime} \pm 1}\left(q_{j}^{\prime \prime}\right) d q_{j} \\
& =\frac{1}{\sqrt{N} \omega_{l}^{2}}\left[A_{j}^{\prime} \int \mathbf{X}_{n_{j}^{\prime}}^{\prime}\left(q_{j}\right) \mathbf{X}_{n^{\prime} \pm 1}\left(q_{j}\right) d q_{j}+A_{j}^{\prime \prime} \int \mathbf{X}_{n_{j}^{\prime}}\left(q_{j}\right) \mathbf{X}_{n^{\prime} \pm 1}^{\prime}\left(q_{j}\right) d q_{j}\right]+O \frac{1}{N}, \text { etc. }
\end{aligned}
$$

The energy for a transition between the electronic states $\mu^{\prime \prime}$ and $\mu^{\prime}$ is $E_{\mu^{\prime \prime} \mu^{\prime}}$ with the corresponding frequency $h \nu=E_{\mu^{\prime \prime} \mu^{\prime}}+\hbar \omega_{l} \sum_{j} \Delta n_{j}$, where $\Delta n_{j}=n_{j}^{\prime \prime}-n_{j}^{\prime}$. For transitions in which no $\Delta n_{j}$ is greater than one, the square of the overlap integral can be written as

$$
\begin{aligned}
& \left|\int \mathbf{X}_{\mu^{\prime} n^{\prime}}^{*} \mathbf{X}_{\mu^{\prime \prime} n^{\prime \prime}} d \mathbf{X}\right|^{2}=\left\{\prod_{j}\left|\int \mathbf{X}_{n_{j}^{\prime}}\left(q_{j}^{\prime}\right) \mathbf{X}_{n_{j}^{\prime}}\left(q_{j}^{\prime \prime}\right) d q_{j}\right|^{2}\right\} \\
& \times\left\{\prod_{l} \frac{\left|\int \mathbf{X}_{n_{l}^{\prime}}\left(q_{l}^{\prime}\right) \mathbf{X}_{n_{l}^{\prime}+1}\left(q_{l}^{\prime \prime}\right) d q_{l}\right|^{2}}{\left|\int \mathbf{X}_{n_{l}^{\prime}}\left(q_{l}^{\prime}\right) \mathbf{X}_{n_{l}^{\prime}}\left(q_{l}^{\prime \prime}\right) d q_{l}\right|^{2}}\right\}\left\{\prod_{k} \frac{\left|\int \mathbf{X}_{n_{k}^{\prime}}\left(q_{k}^{\prime}\right) \mathbf{X}_{n_{k}^{\prime}-1}\left(q_{k}^{\prime \prime}\right) d q_{k}\right|^{2}}{\left|\int \mathbf{X}_{n_{k}^{\prime}}\left(q_{k}^{\prime}\right) \mathbf{X}_{n_{k}^{\prime}}\left(q_{k}^{\prime \prime}\right) d q_{k}\right|^{2}}\right\} .
\end{aligned}
$$

The summations over $l$ and $k$ are both extended over all lattice oscillators. For the indices $\mathbf{y}$ and $\lambda$, the summations can be replaced by integrations. When taking into account only terms below order $\frac{1}{\sqrt{N}}$ one obtains

$$
\prod_{j}\left|\int \mathbf{X}_{n_{j}^{\prime}}\left(q_{j}^{\prime}\right) \mathbf{X}_{n_{j}^{\prime}}\left(q_{j}^{\prime \prime}\right) d q_{j}\right|^{2}=\sum_{t=0}^{\infty} \frac{\left(-S_{+}-S_{-}\right)^{t}}{t !}=\exp \left[-\left(S_{+}+S_{-}\right)\right],
$$

with

$$
S_{ \pm}=S\left\{\begin{array}{l}
\bar{n}(T)+1 \\
\bar{n}(T)
\end{array}\right.
$$

the thermal average of the vibrational quantum number $\bar{n}(T)=\left(\exp \left(\hbar \omega_{l} / k T\right)-1\right)^{-1}$ and the constant integral

$$
S=\frac{\hbar \nu_{a}}{2 \omega_{l}^{3}} \sum_{y} \int\left(A_{\mathbf{y} \lambda}^{\prime \prime} A_{\mathbf{y} \lambda}^{\prime}\right)^{2} d \mathbf{y} .
$$

The coupling constant $S$ was later named Huang-Rhys parameter.

\subsection{Exciton-phonon coupling in Raman spectra}

The coupling between phonons and excited carriers is reflected in the relative intensities of the overtones to the fundamental Raman band. The correlation between 
Raman spectra and the exciton-phonon coupling can be deduced from works from Tannor and Heller[80], Albrecht[81] and others[82, 83]. The Kramer-HeisenbergDirac theory is valid for Franck-Condon displacements. For Raman scattering, the relevant part of the Kramer-Heisenberg-Dirac formula arises from second-order perturbation theory and is written as

$$
\begin{aligned}
\alpha_{f i}= & \sum_{k} \sum_{n}\left[\frac{\left\langle\chi_{1 f}|\mu| \chi_{k n}\right\rangle\left\langle\chi_{k n}|\mu| \chi_{1 i}\right\rangle}{\hbar\left(\omega_{1 i}+\omega_{I}-\omega_{k n}-i \Gamma_{k}\right)}\right. \\
& +\frac{\left\langle\chi_{1 f}|\mu| \chi_{k n}\right\rangle\left\langle\chi_{k n}|\mu| \chi_{1 n}\right\rangle}{\hbar\left(\omega_{1 i}+\omega_{s}-\omega_{k n}-i \Gamma_{k}\right)}
\end{aligned}
$$

with $\mu=\mathbf{D} \cdot \mathbf{e}$. The sum over $k$ and $n$ represents a sum over all Born-Oppenheimer electronic states and vibrational levels. $\chi_{k n}$ then refers to all intermediate vibrational states that can be excited. $\chi_{1 i}$ and $\chi_{1 f}$ are the initial and final vibrational states of the ground electronic state. $\hbar \omega_{1 i}$ is the energy of the ground electronic state, $\hbar \omega_{k n}$ is the energy of the intermediate state, $\hbar \omega_{I}$ is the energy of the incident radiation and $\hbar \omega_{s}$ the energy of the scattered radiation. $\Gamma_{k}$ is a phenomenological lifetime factor. The intensity $I^{i \rightarrow f}$ into a particular mode $f$ is proportional to $\omega_{I} \omega_{s}^{3}\left|\alpha_{f i}\right|^{2}$.

For a single electronic state $\left|\phi_{i}\right\rangle=\mu\left|\chi_{1 i}\right\rangle,\left|\phi_{f}\right\rangle=\mu\left|\chi_{1 f}\right\rangle$ and $\omega^{\prime}=\omega_{1 i}+\omega_{I}$ can be written and equation 5.18 has the simpler form

$$
\alpha_{f i}=\sum_{n} \frac{\left\langle\phi_{f} \mid \chi_{n}\right\rangle\left\langle\chi_{n} \mid \phi_{i}\right\rangle}{\hbar\left(\omega^{\prime}-\omega_{n}+i \Gamma_{k}\right)} .
$$

This expression can be transformed to the time domain, where harmonic potentials can be treated analyticly[80] and then reads as

$$
\alpha_{f i}=\frac{i}{\hbar} \int_{0}^{\infty}\left\langle\phi_{f} \mid \chi_{i}(t)\right\rangle \exp \left(\left(i \omega^{\prime}-\Gamma\right) t\right) d t
$$

with $\left|\chi_{i}(t)\right\rangle \equiv \exp \left(-i H_{e x} t / \hbar\right)\left|\chi_{i}\right\rangle$ and the excited electronic state Hamiltonian $H_{e x}$. The displacement of the excited-state equilibrium relative to the ground-state equilibrium can be written as $\Delta$. One can then calculate the overlap between a moving wave packet and the final state of interest and obtain the Raman amplitude.

$$
\begin{aligned}
I_{n}= & \left\langle\phi_{n} \mid \phi_{0}(t)\right\rangle \\
= & \exp \left\{-\Delta^{2} / 2[1-\exp (-i \omega t)]-i \omega t / 2\right\}[1-\exp (-i \omega t)]^{n} . \\
& \times \frac{(-1)^{n} \Delta^{n}}{\left(2^{n} n !\right)^{1 / 2}} \cdot \exp \left(-i \omega_{t}\right)
\end{aligned}
$$


Chapter 5. Exciton-LO-phonon coupling in CdSe nanorods

When comparing this expression with equation 5.15 one finds that $S=\frac{1}{2} \Delta^{2}$ applies. Based on this time-dependend overlap integral, the integrated Raman intensities can be calculated. According to Albrecht, the Raman scattering cross section for an order- $n$ phonon process can be written in the form

$$
\left|R^{n}(\omega)\right|^{2}=\mu^{4}\left|\sum_{m=0}^{\infty} \frac{\langle n \mid m\rangle\langle m \mid 0\rangle}{E_{i j}+n \hbar \omega_{\mathrm{LO}}-\hbar \omega+i \hbar \Gamma}\right|^{2}
$$

[81]. In this notation $\hbar \omega$ is the the incident photon energy, the excited state energy is written as $E_{i j}$, the electronic transition dipole moment is $\mu$, the homogeneous linewidth is $\Gamma$ and $m$ is the intermediate state. The Franck-Condon overlap is

$$
\langle n \mid m\rangle=\left(\frac{n !}{m !}\right)^{\frac{1}{2}} \exp \left(-\frac{1}{2} \Delta^{2}\right) \Delta^{n-m} L_{m}^{n-m}\left(\Delta^{2}\right),
$$

with the Laguerre polynomial $L$. The ratio of two different cross sections (Equation 5.22) yields, together with Equation 5.23, an expression that depends on the integrated Raman intensities and $\Delta$. Measuring the fundamental Raman band and an overtone or two different overtones of the system of interest then allows the numerical estimation of $\Delta$ and $S$. It is adjuvant to measure the two bands in a single spectrum, as this makes the normalization easier. For practical reasons one usually measures the fundamental Raman band and its first overtone, because they are most intense. 
5.3 Huang-Rhys parameter of CdSe nanorods

\subsection{Huang-Rhys parameter of CdSe nanorods}

The Fröhlich interaction between the longitudinal optical phonons and an exciton can be approximated by the interaction of the longitudinal optical phonon with a static charge distribution and then discussed within the framework presented above[83]. Figure 5.1 displays a Raman spectrum of CdSe nanorods with $4 \mathrm{~nm}$ diameter and $25 \mathrm{~nm}$ length with fundamental Raman band and first overtone. The contributions to the spectrum were discussed in Chapter 4. Relevant for the excitonphonon coupling are the LO related bands. As presented in Chapter 4, the contributions from the LO can be fitted with a Lorentzian function and separated from the complete Raman spectrum.

The Raman cross sections required to evaluate equation 5.22 are the areas of the corresponding Lorentzian functions. The ratio of equation 5.22 for $n=2$ and $n=1$ is an equation for $\Delta$ that can be evaluated numerically for large numbers of intermediate states $m$. The required constants are the exciting photon frequency, which are $514.5 \mathrm{~nm}$-photons, the LO frequency, which is taken as the position of the Lorentzian fit to the LO Raman band, the exciton energy and the corresponding phenomenological linewidth. The values for the excitonic transition can be obtained from spectral hole burning measurements on the CdSe nanorods[14]. The exciton resonance is located at $\lambda_{\mathrm{ex}}=590 \mathrm{~nm}$ and the linewidth ranges between $\Gamma=10-20 \mu \mathrm{eV}$. Krauss and Wise investigated exciton-phonon coupling in $\mathrm{PbS}$ nanocrystals[84]. They systematically varied the linewidth in a numerical model and monitored the influence on the coupling strength. They found the influence of $\Gamma$ on $S$ to be small. As their discussion is general, these findings also apply to CdSe nanostructures. Thus, $\Gamma=20 \mu \mathrm{eV}$ is used. Figure 5.2 displays the Huang-Rhys parameter versus the ratio of the first overtone to the fundamental Raman band.

The Huang-Rhys parameter scales with the ratio and is almost linear up to a ratio of 1 . Using the measured ratio of the Raman cross sections and the parameters mentioned above results in $S=0.038$ for CdSe nanorods of $4 \mathrm{~nm}$ diameter and $25 \mathrm{~nm}$ length.

The Huang-Rhys parameter for an exciton in bulk material can be deduced from the exciton-phonon-matrix element by using a hydrogen-like wave function for the exciton. Nomura and Kobayashi explicitly calculate the coupling strength for a 


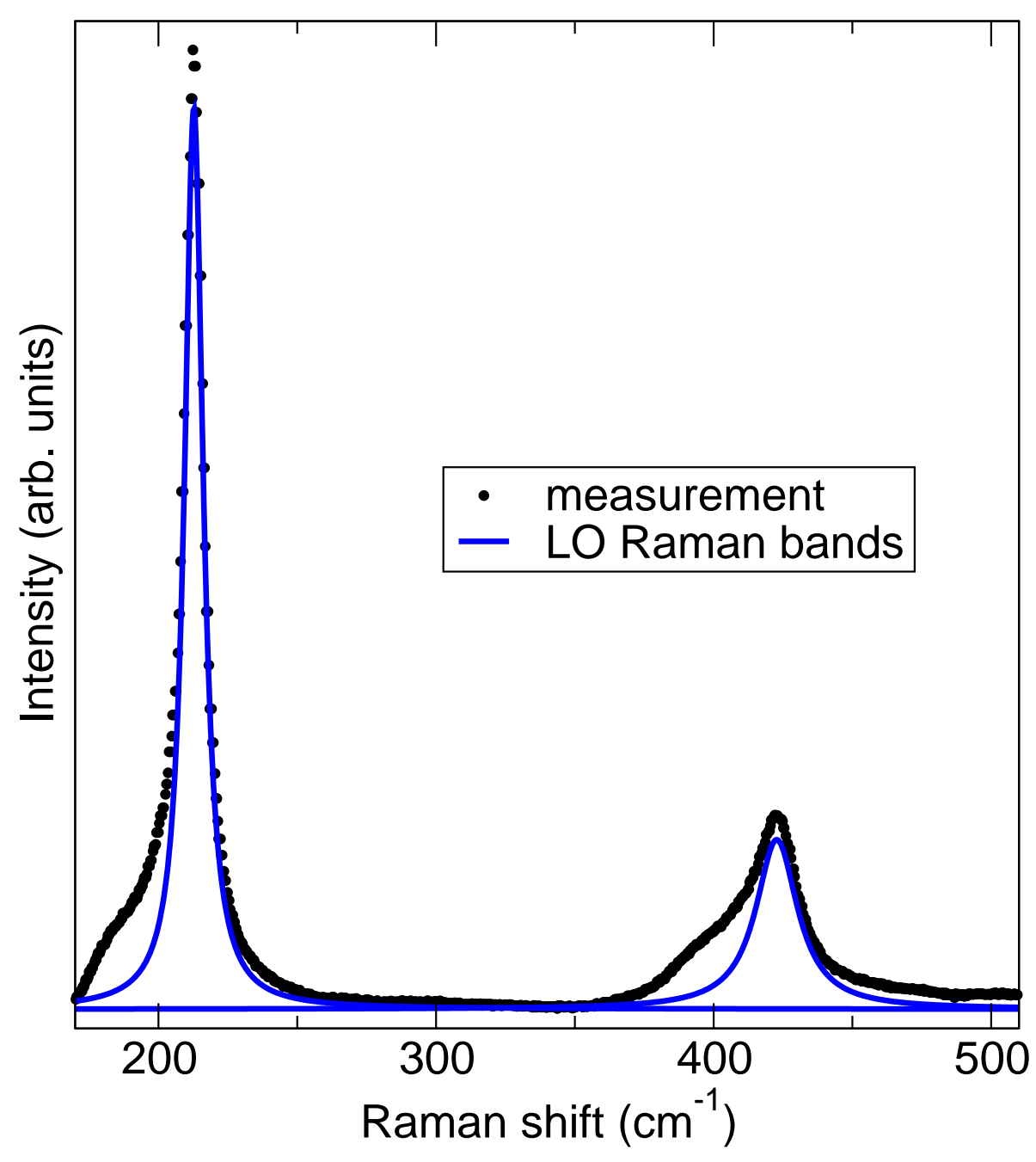

Figure 5.1: Raman spectrum of CdSe nanorods with $4 \mathrm{~nm}$ diameter and $25 \mathrm{~nm}$ length with underlying LO bands. 


\subsection{Huang-Rhys parameter of CdSe nanorods}

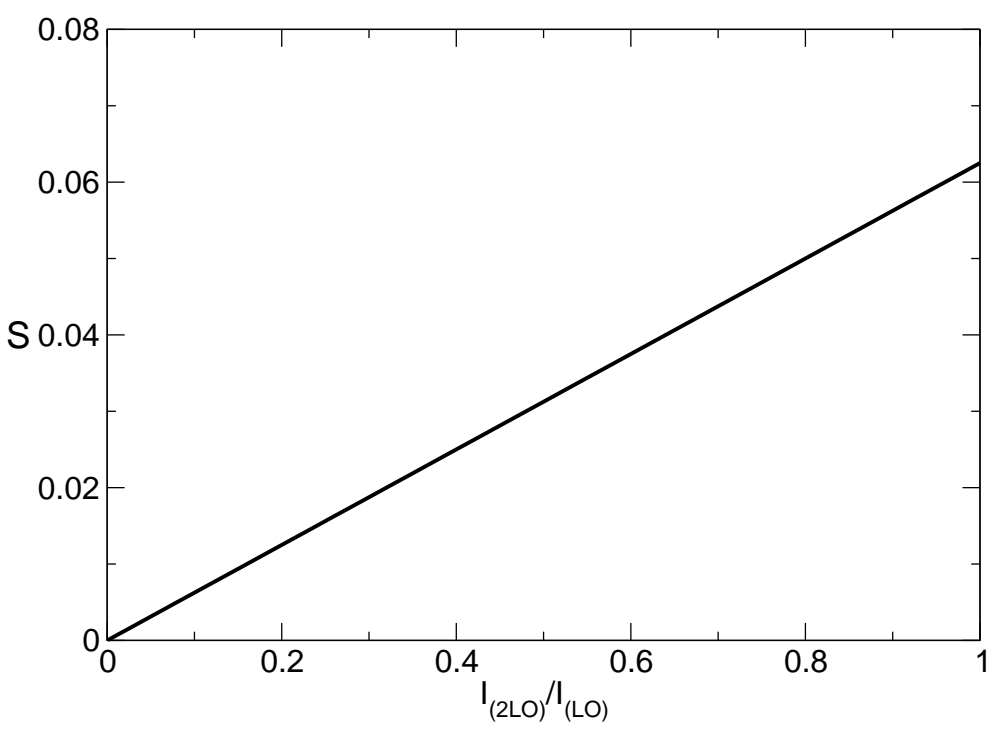

Figure 5.2: Huang-Rhys parameter versus ratio of the integrated intensities of second order and fundamental Raman band.

Wannier exciton in bulk CdSe. It reads as[85]

$$
\begin{aligned}
S= & \frac{f_{0}^{2}}{2 \pi^{2}\left(\hbar \omega_{L O}\right)^{2}} \int_{0}^{\infty} d k|\nu(k)|^{2} \\
= & \frac{f_{0}^{2}}{32 \pi^{2} a_{B}\left(\hbar \omega_{L O}\right)^{2}} \\
& \times \frac{\left(m_{1}^{*}-m_{2}^{*}\right)^{2}\left(5 m_{1}^{* 2}+14 m_{1}^{*} m_{2}^{*}+5 m_{2}^{* 2}\right)}{m_{1}^{*} m_{2}^{*}\left(m_{1}^{*}+m_{2}^{*}\right)^{2}} .
\end{aligned}
$$

$f_{0}$ is the Fröhlich interaction coupling constant, $m_{1}^{*}$ and $m_{2}^{*}$ are the effective masses of an electron and a hole. $S$ strongly depends on the choice of the effective masses. Reasonable choices of $m_{2}^{*}$ for CdSe result in $S=0.38$ or $S=0.79$. These are by more than one order of magnitude larger than the measured value for the $4 \mathrm{~nm}$ diameter and $25 \mathrm{~nm}$ length CdSe nanorods.

The interaction of excitons with longitudinal optical phonons differs between nanocrystals and bulk material. In nanocrystals all relevant states are discrete. In bulk material there is a continuum state as a final state. In nanocrystals there is no such final state and scattering can only occur in first order when the energy separations of the discrete states coincide with the LO energy. Furthermore, the exciton-LO interaction is quenched in nanocrystals $[85,86]$. The exciton-phonon coupling constant 
Chapter 5. Exciton-LO-phonon coupling in CdSe nanorods

Table 5.1: Longitudinal optical phonon and overtone data for different geometries.

\begin{tabular}{c|ccccc} 
sample dimesion $\mathrm{nm}^{2}$ & $8 \times 80$ & $7 \times 35$ & $6 \times 25$ & $4 \times 25$ & $3.5 \times 20$ \\
\hline 2LO area (arb. units) & 4.51 & 2.95 & 4.25 & 5.35 & 12.0 \\
LO area (arb. units) & 11.76 & 7.55 & 10.9 & 11.76 & 14.3 \\
2LO/LO & 0.38 & 0.39 & 0.41 & 0.45 & 0.83 \\
S & 0.024 & 0.025 & 0.029 & 0.038 & 0.053
\end{tabular}

depends on the difference of the wave functions between an electron and a hole and quenching changes the wave functions differently [85]. In bulk material, the excitons do not experience any confinement and the coupling is dominated by the Coulomb interaction. For decreasing crystal size, the Coulomb interaction becomes less and less important and the difference in the effective masses of the electron and hole become smaller due to the confinement of the corresponding wave functions. This results in a decrease of the Huang-Rhys parameter.

The nanorod size-dependence on the exciton-phonon coupling strength can be investigated by evaluating the overtone-to-fundamental ratios for various nanorod geometries. The size-tunability of the nanorods then allows a systematic investigation.

Figure 5.3 displays an exemplary Raman spectrum of a different nanorod geometry, CdSe nanorods of $7 \mathrm{~nm}$ diameter and $35 \mathrm{~nm}$ length with fundamental Raman band and first overtone. The ratio of the integrated intensities of the first overtone to the fundamental Raman band differs from the nanorods of $4 \mathrm{~nm}$ diameter and $25 \mathrm{~nm}$ length. The overtone intensity is much smaller while the fundamental LO Raman band is of similar shape. Due to the quasi-linear dependence on the overtone to fundamental ratio, this results in a reduced Huang-Rhys parameter of $S=0.025$. Even though the diameter of the nanorod is increased compared to the $4 \mathrm{~nm}$ diameter nanorod, the Huang-Rhys parameter further differs from the bulk value. This behavior is systematic. The integrated intensities of the relevant Raman bands and the Huang-Rhys parameter for various nanorod geometries are summarized in table 5.1 .

The coupling strength increases for decreasing nanorod dimesions. This behavior seems to depend mostly on the nanorod diameter. Two nanorods with the same length, but different diameters, nanorods of $4 \mathrm{~nm}$ diameter and $25 \mathrm{~nm}$ length and nanorods of $6 \mathrm{~nm}$ diameter and $25 \mathrm{~nm}$ length have a very different Huang-Rhys parameter, while nanorods with a similar diameter but different lengths, nanorods of $7 \mathrm{~nm}$ diameter and $35 \mathrm{~nm}$ length and nanorods of $8 \mathrm{~nm}$ diameter and $80 \mathrm{~nm}$ length have almost the same Huang-Rhys parameter. Thus, a diameter dependence seems probable. The Huang-Rhys parameter versus the nanorod diameter is plotted in Figure 5.4 


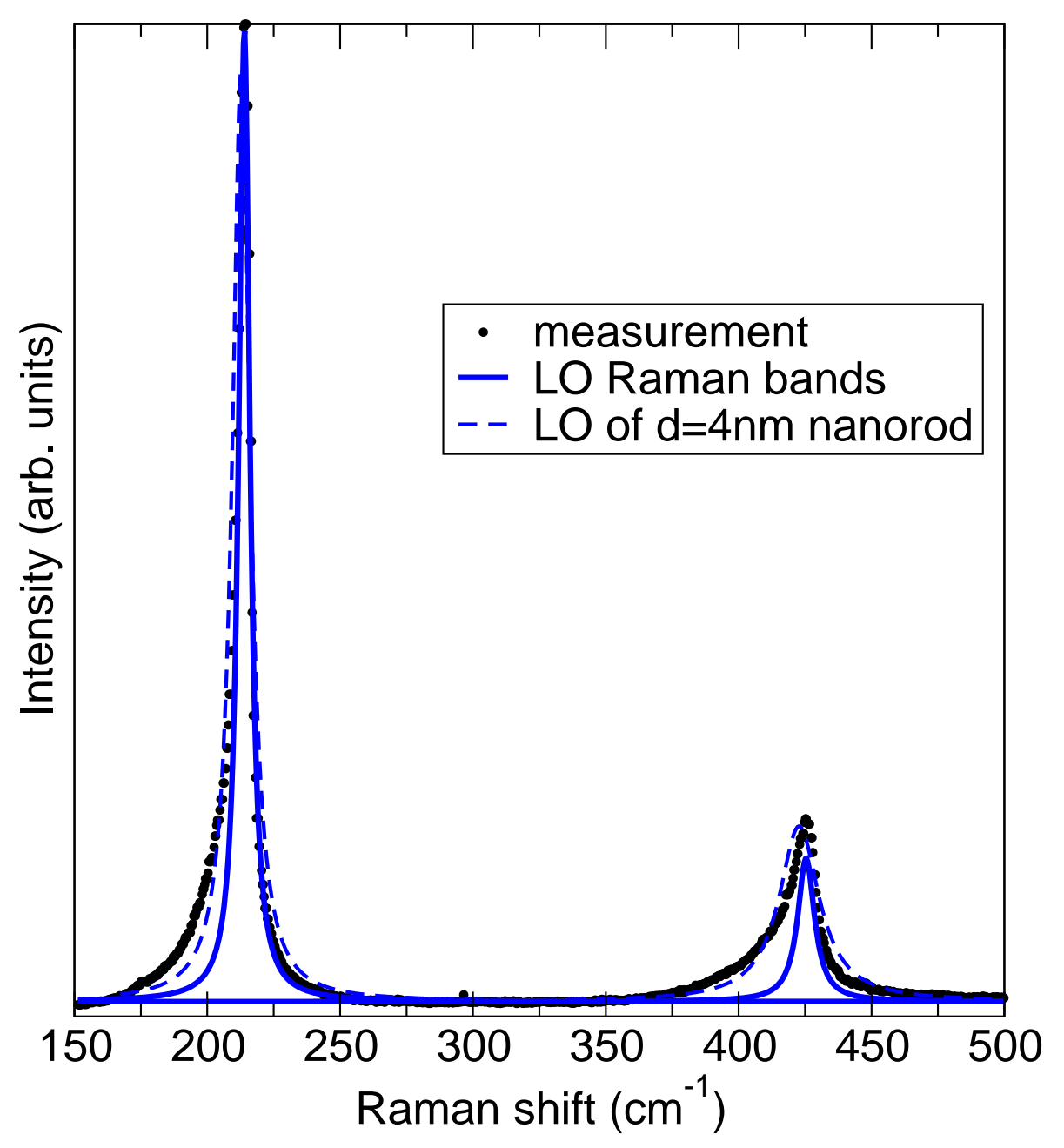

Figure 5.3: Raman spectrum of CdSe nanorods with $7 \mathrm{~nm}$ diameter and $35 \mathrm{~nm}$ length at liquid helium temperatures with underlying LO bands. The LO bands from nanorods of $4 \mathrm{~nm}$ diameter and $25 \mathrm{~nm}$ length are included for comparison 
Chapter 5. Exciton-LO-phonon coupling in CdSe nanorods

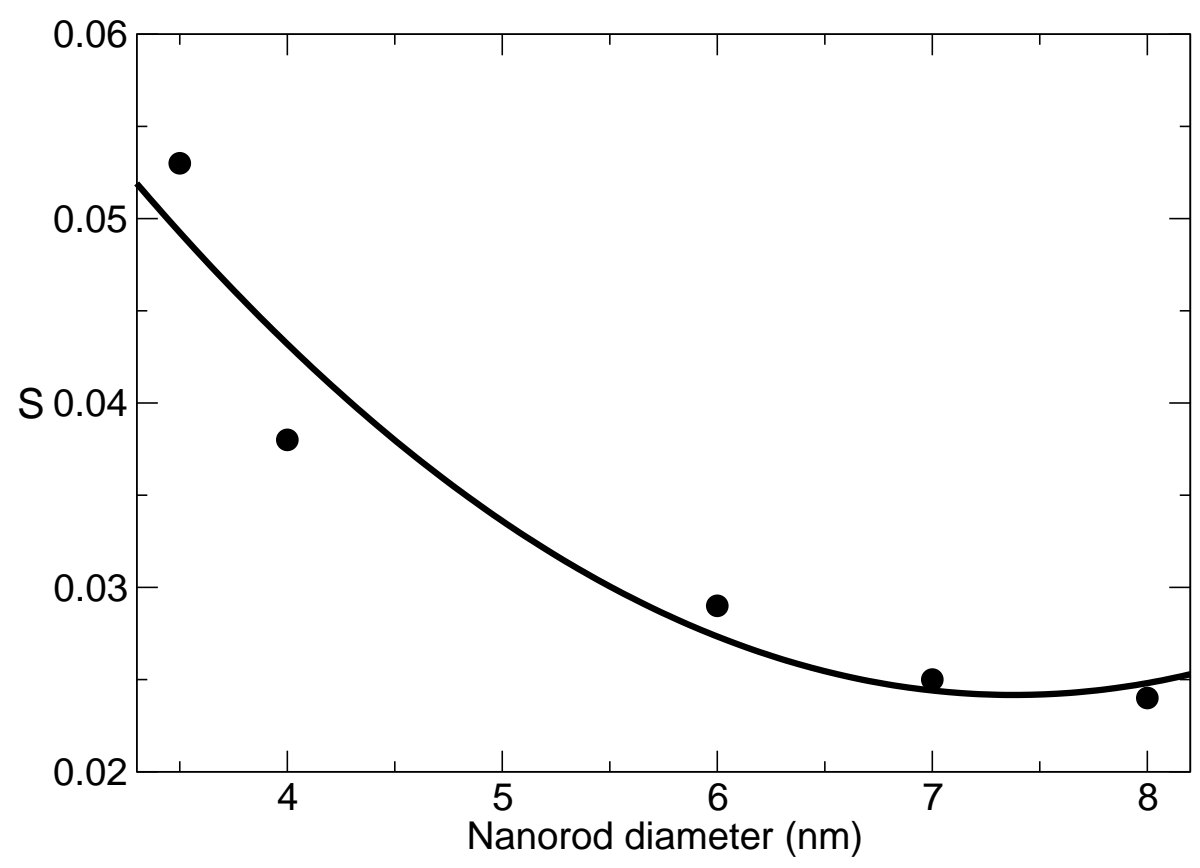

Figure 5.4: Diameter dependent Huang-Rhys parameter. The solid line is a guide to the eye.

The diameter dependent behavior is similar to results for CdSe nanocrystals of $2-3 \mathrm{~nm}$ diameter reported by Scamario et al. [87]. There, the coupling strength also increases with decreasing diameter in a very similar way as the measurements on the nanorods show. Even the largest Huang-Rhys parameter for the smallest structures, however, is much smaller than the Huang-Rhys parameter in bulk material. This indicates the influence of two autonomous effects. One that results in an overall reduced coupling strength for small crystals and one that slightly increases the coupling strength for decreasing radii in nanocrystals. Nomura and Kobayashi calculate the dependence of the Huang-Rhys parameter on the diameter of CdSe and GaAs microcrystallites by applying "free-standing" and "rigid" boundary conditions and considering valence-band mixing[85]. As in the experimental results, they find a reduced coupling strength relative to bulk, but an increase in the coupling strength for decreasing diameters. A diagram of their results is shown in Figure 5.5.

The course of the coupling strength versus diameter dependence starting from bulk decreases for decreasing diameters, has a minimum value for a certain diameter and then rises again for further decreasing diameters, around $70 \AA$ for CdSe spheres. The latter effect is due to an increase in couplings to higher frequency phonons with the reduction of the nanocrystal diameter. The former effect is related to a decrease of 


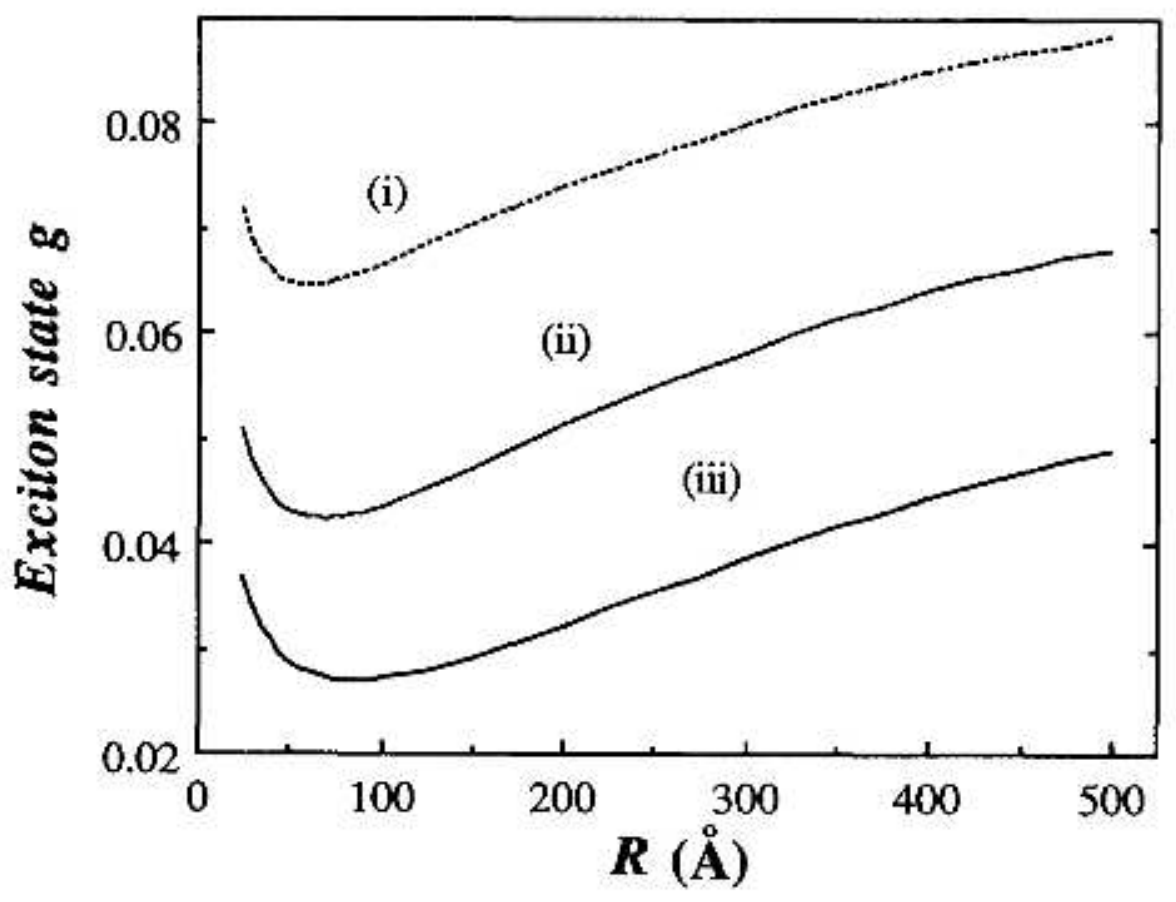

Figure 5.5: Calculated size dependence of the Huang-Rhys parameter for $\left(s, S_{3 / 2}\right.$ exciton states of CdSe with the inverse averaged effective hole masses $\gamma_{1}^{-1}=1.0$ (i), 0.68 (ii) and 045 (iii). Taken from reference [85].

the influence of the Coulomb interaction, as mentioned above. The observed similarity between the Huang-Rhys parameter of CdSe nanorods and spherical nanocrystals can be explained by fluctuations in the confinement potential along the nanorod axis. The diameter of the nanorods varies slightly along the nanorod length, as shown in Chapter 2. The nanorod excitons then become weakly localized in these thickness fluctuations and experience a rather anisotropic then ideal 1D-confinement, i.e. are effectively of the same dimensions as excitons in spherical structures. As for the luminescent properties (see Chapter 2), the nanorod diameter is the most important geometrical parameter for the excitons. A futher increase of the diameter should lead to an increase of the coupling strength. Then, the length should also contribute to the size-dependence, as the excitons become less localized.

In this chapter the geometry dependence of the exciton-phonon coupling strength was investigated. The nanorods behave like spherical particles. This is due to the confinement of the excitons between diameter fluctuations of the nanorod. As for the spherical particles, the coupling strength is much lower than in bulk material 
Chapter 5. Exciton-LO-phonon coupling in CdSe nanorods

because of a decrease of the influence of the Coulomb interaction in small particles. The coupling strength rises for decreasing diameters due to an increase in couplings to higher frequency phonons. 


\section{Chapter 6}

\section{The Radial breathing mode in CdSe nanorods}

The influence of the nanorod dimensions on the fundamental Raman band was investigated in Chapter 4 and a diameter- and an aspect ratio dependence was found for the two main contributions. A diameter dependence was also found for the Huang-Rhys parameter, presented in Chapter 5. In this chapter another diameter dependent Raman active mode is presented. A fully symmetrical, breathing-like vibrational mode, whose frequency is highly diameter sensitive, is present in the nanorods. The measurement of this mode can be used to estimate the nanorod diameter and diameter fluctuations.

\subsection{Motivation}

As seen in the previous chapters, Raman spectroscopy is a versatile method to gain information on various properties of CdSe nanorods. As it is non-destructive it can easily be combined with other methods. In another low-dimensional system, the carbon nanotubes, Raman spectroscopy is frequently used to gain structural information. There, the radial breathing mode (RBM) corresponds to the atomic vibration of the carbon atoms in-phase in the radial direction, as if the tube was breathing. Figure 6.1 displays a displacement pattern of such a mode.

The frequency of the RBM follows a $1 / d$ diameter dependence and the diameter of carbon nanotubes is determined by their chiral indices. The dependence of the RBM frequency on the nanotube diameter is frequently used to determine the tube diameters and the diameter distribution in a nanotube sample from Raman scattering[88]. 


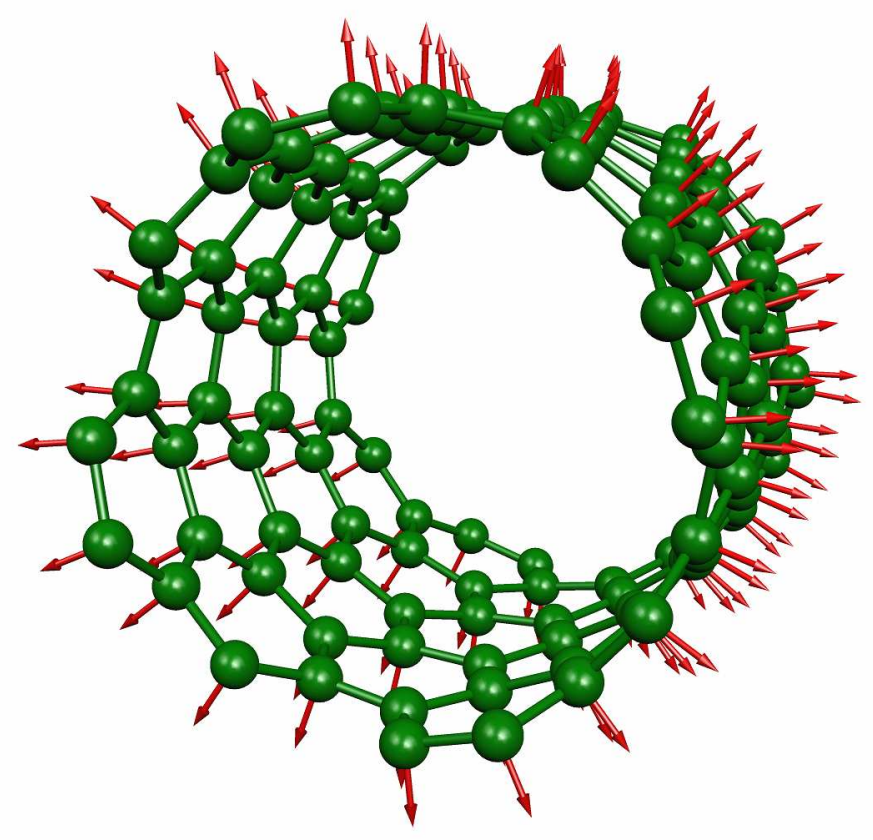

Figure 6.1: Displacement pattern of the RBM of a carbon nanotube. The arrows show the phonon eigenvector. The RBM leads to a periodic increase and decrease of the tube diameter as shown by the wire model of the tube. Image courtesy of María Machón.

The chiral indices can be estimated by resonant measurements of the RBM. The Raman signal intensity increases strongly when the excitation energy approaches an allowed optical transition. If the incoming or scattered light matches the transition energy, this is called a resonance, and the intensity is at maximum. Measuring the Raman intensity as a function of the incident photon energy allows the determination of the transition energies in carbon nanotubes. The chiral indices can then be retrieved with the resonance energy and the RBM frequency by using the so-called Kataura plot[88].

The CdSe nanorods have, connatural to the nanotubes, an axially symmetric geometry and are of comparable size. 


\subsection{Linear elasticity theory}

In principle, a nanorod can be approximated as a solid cylinder with the length $l$ and the radius $R$. In case of $l$ beeing much larger than $R$, the frequency of a breathing-like vibration can be deduced from linear elasticity theory[89, 90]. Linear elasticity implies the continuum hypothesis. The fundamental assumptions of linear elasticity are: infinitesimal strains and linear relationships between stress and strain. In linear elasticity, the radial displacement of a solid cylinder has the form

$$
\mathbf{U}_{r m}=\mathbf{u}_{r m}(r) \exp \left(-i \omega_{r m} t\right)
$$

with

$$
\mathbf{u}_{r m}(r)=u(r) \hat{\mathbf{r}},
$$

where $u(r)$ is the radial displacement of the nanorod, $\hat{\mathbf{r}}$ the unit vector in the readial direction. The governing equation for the radial displacement is

$$
\frac{d^{2}}{d r^{2}}+\frac{1}{r} \frac{d u}{d r}-\frac{u}{r^{2}}+h^{2} u=0
$$

with

$$
h=\omega_{r m} \sqrt{\frac{\rho(1+\nu)(1-2 \nu)}{E(1-\nu)} .}
$$

$E$ is the Young's modulus, $\rho$ the density and $\nu$ the Poisson's ratio of the nanorod. Following the approach by $\mathrm{Hu}$ et al.[89], the stress is considered zero at the surface of the nanorod. This leads to the boundary condition

$$
\left[\frac{d u}{d r}+\frac{\nu}{1-\nu} \frac{u}{r}\right]_{r=R}=0 .
$$

Solving equation 6.2 subject to the boundary condition equation 6.5 yields

$$
u(r)=\frac{J_{1}\left(\frac{\tau r}{R}\right)}{J_{1}(\tau)} .
$$

The resonant frequencies are

$$
\omega_{r m}^{(n)}=\frac{\tau_{n}}{R} \sqrt{\frac{E(1-\nu)}{\rho(1+\nu)(1-2 \nu)}},
$$

where $\tau_{n}$ is the $n$-th root of the eigenvalue equation

$$
\tau J_{0}(\tau)=\frac{1-2 \nu}{1-\nu} J_{1}(\tau) .
$$


Chapter 6. The Radial breathing mode in CdSe nanorods

The square root in equation 6.7 depends only on the material properties.

Thonhauser and Mahan find a similar dependence for Si[111] nanowires treated with a Stillinger-Weber-type model for isotropic elasticity theory[91]. There, the radial breathing mode frequency is

$$
\omega=\frac{\nu_{l}}{R} \sqrt{\frac{8(1-r)}{2-r}}
$$

with $r=\frac{\mu}{\lambda+2 \mu}$, the longitudinal speed of sound $\nu_{l}$ and the elastic constants $(\lambda, \mu)$. Both models result in a $1 / d$ diameter dependence for the radial breathing mode frequency, but with different material dependent constant factors. Using literature values for the constant factors in equation 6.7 results in $C=82.8 \mathrm{~cm}^{-1} \mathrm{~nm}$ for the $\omega_{\mathrm{RBM}}=C / d$-dependence of the radial breathing mode frequency[28, 92].

\subsection{Ab-initio calculations}

The linear elasticity model treats the nanorods as axially symmetric cylinders. The wurtzite CdSe nanorods have a hexagonal cross-section. To account for this and to take into account the microscopic structure, microscopic ab-initio calculations of CdSe nanorods rather linear elasticity approximations are appropriate. The framework for the calculations were presented in Chapter 3. By using programs implemented in SIESTA, the phonons can be calculated. Beyond the transverse and longitudinal phonons, there are numerous other vibrational modes with different symmetries present in the nanorods. Some of them exist only in a fraction of the calculated geometries. One prominent mode can be found in all calculated nanorods. In this vibration, the atoms move almost radially. This mode is fully symmetric. An exemplary displacement pattern of a $1.4 \mathrm{~nm}$ diameter nanorod is displayed in Figure 6.2.

This mode is the atomic counterpart to the radial breathing mode of a solid cylinder treated above with linear elasticity. When calculating larger nanorods the frequency of this radial breathing mode decreases. The obtained RBM frequencies range from $40-160 \mathrm{~cm}^{-1}$ for the calculated diameter range of $2.6-0.6 \mathrm{~nm}$. As in linear elasticity, the frequencies show a $1 / d$ dependence on the diameter. The frequency versus nanorod diameter dependence can be fitted with a const./ $d$-function. The constant factor, however, is larger than the one obtained from elasticity theory. 


\subsection{Raman measurement of the radial breathing mode}

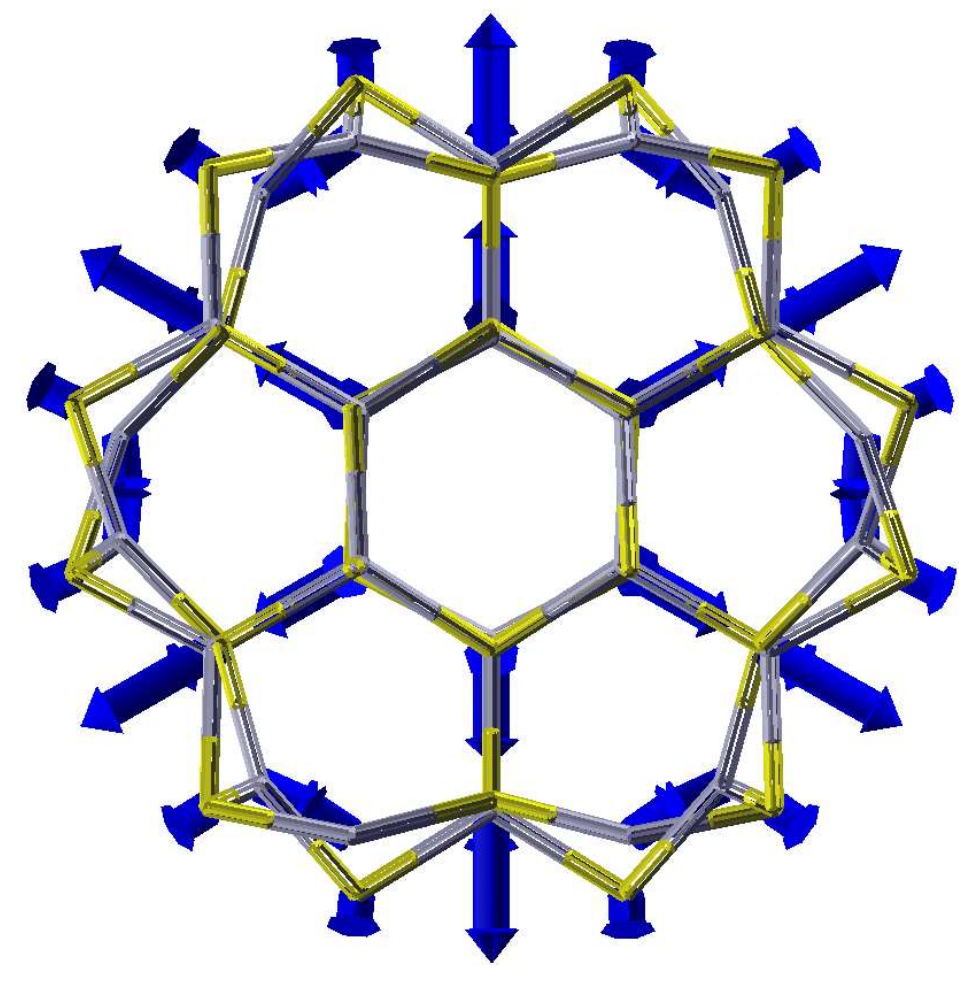

Figure 6.2: Displacement pattern of the radial breathing mode of a $1.4 \mathrm{~nm}$ diameter CdSe nanorod.

\subsection{Raman measurement of the radial breathing mode}

Following the linear elasticity and ab-initio calculations, the radial breathing mode has to fullfill two criteria: its frequency has to depend on the nanorod diameter and follow a $1 / d$ diameter dependence and it has to independent of the nanorod length. The size tunability of the nanorods allows a systematic investigation of such a mode. Nanorods with different diameters can be grown to monitor the diameter dependence of the RBM and nanorods with the same diameter but different lengths can be synthesized to verify the pure radial dependence of the mode. An approximation of the RBM frequency for a given nanorod diameter can be obtained by entering the diameter in the linear elasticity model (equation 6.7), or by taking the const./ $d$ function fitted to the frequencies from the $a b$-initio calculations and entering the 
Chapter 6. The Radial breathing mode in CdSe nanorods

diameter there. For example, the radial breathing mode frequency of a nanorod of $4 \mathrm{~nm}$ diameter is expected to be around $20 \mathrm{~cm}^{-1}$. For many common Raman set-ups, this is almost too close to the laser line. This frequency range is accessible with the set-up presented in Chapter 3, when making certain modifications. In the disordered nanorod conglamorates, the Raman part of the scattered light is scattered in all directions. The Rayleigh scattered light of the flat Si substrate, which is the main part of the Rayleigh scattered light, obeys the law of reflection. The angle at which the incident light falls on the surface equals the angle at which it is reflected. When the sample is tilted one can avoid this part of the scattered light to be collected by the imaging optics. The pre-monochromators can be used the shadow parts of the CCD area. This can be used to further suppress the Rayleigh scattered light at the cost of a reduced spectral window. In Figure 6.3 a Raman spectrum acquired this way is shown. It displays the low-frequency regime of the Raman spectrum of CdSe nanorods with $4 \mathrm{~nm}$ diameter and $25 \mathrm{~nm}$ length.

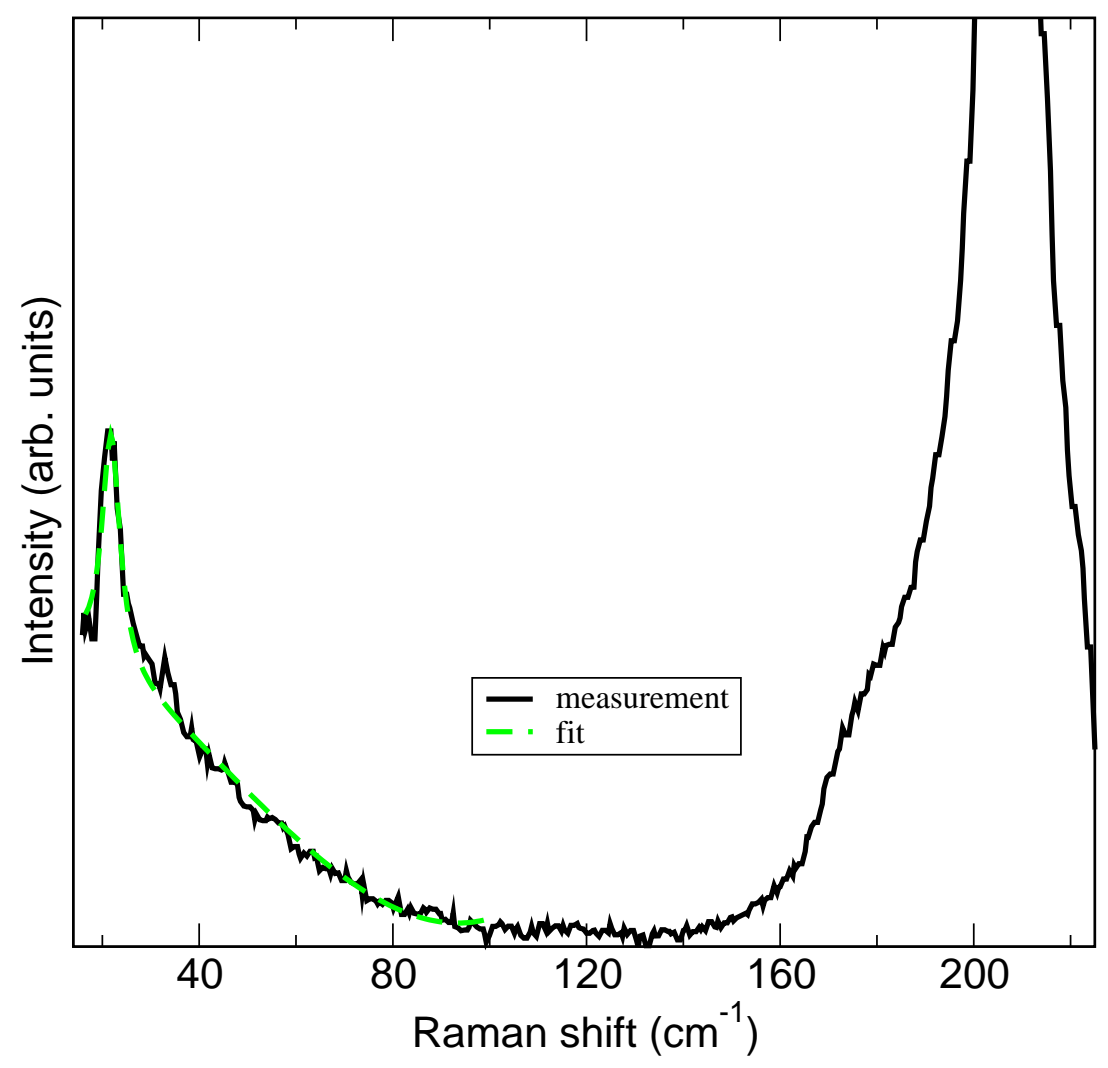

Figure 6.3: Raman spectrum from CdSe nanorods of $4 \mathrm{~nm}$ diameter and $25 \mathrm{~nm}$ length in the low-frequency regime.

The spectrum still has a strong background in the regime between $20 \mathrm{~cm}^{-1}$ and 


\subsection{Raman measurement of the radial breathing mode}

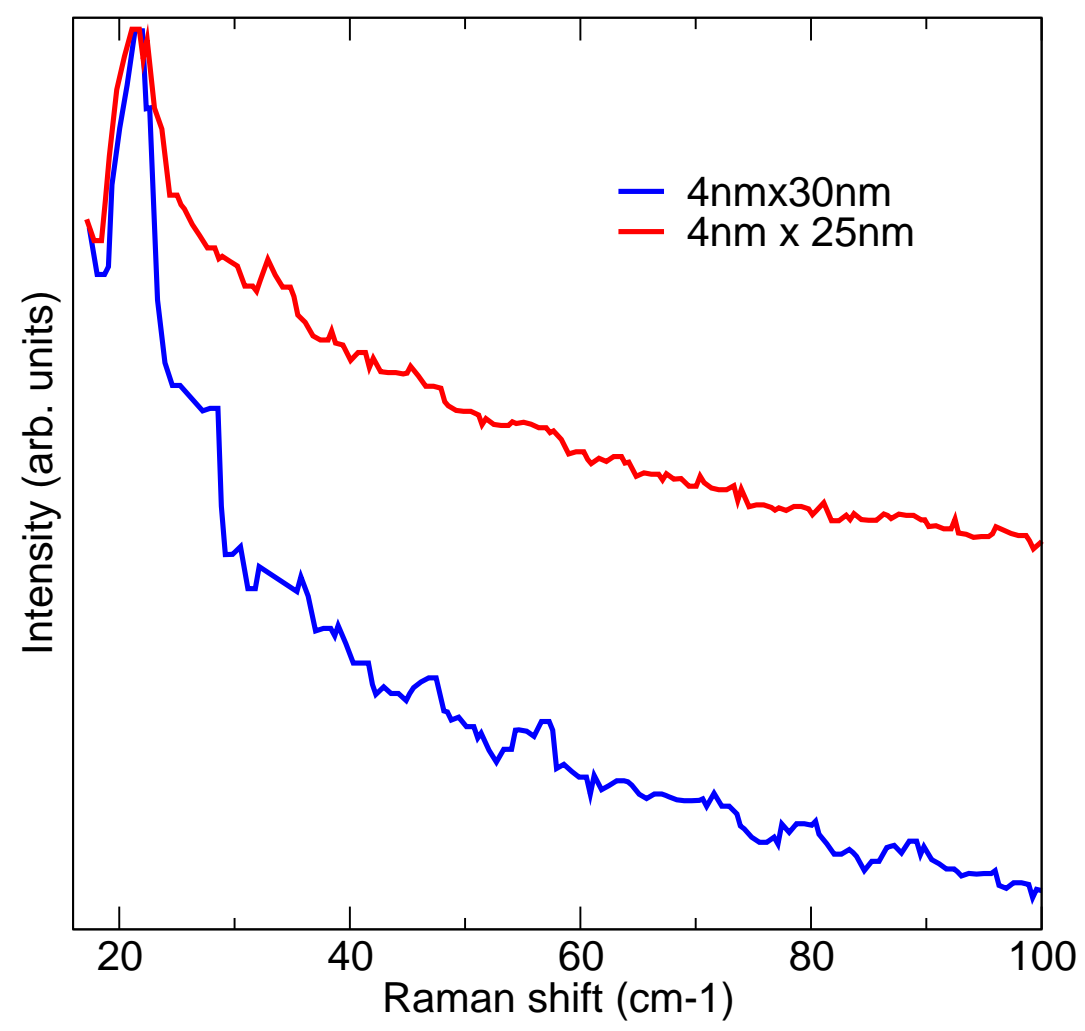

Figure 6.4: Raman spectra from CdSe nanorods of $4 \mathrm{~nm}$ diameter at liquid helium temperature.

$100 \mathrm{~cm}^{-1}$. This is due to elastically scattered laser light of the $514 \mathrm{~nm}$-line of the $\mathrm{Ar}^{+}$-laser. The backround can be fitted with a cubic polynomial. The most prominent feature in the spectrum is the fundamental Raman band around $200 \mathrm{~cm}^{-1}$. A further Raman band can be identified at lower frequencies around $20 \mathrm{~cm}^{-1}$. This is the expected RBM frequency for nanorods of this diameter. This mode can be fitted with a single Lorentzian function.

In case of this mode beeing the RBM, its frequency is independent of the nanorod length. The low-frequency spectrum of a second sample, CdSe nanorods of $4 \mathrm{~nm}$ diameter and $30 \mathrm{~nm}$ length is displayed in Figure 6.4.

Included in the figure is the spectrum from the nanorod sample of the same diameter but with $25 \mathrm{~nm}$ length. The frequency of the RBM is, within the experimental error, the same for both samples, a length dependence of the modes' frequency can be excluded.

The RBM frequency is expected to be diameter dependent. Spectra from two further 
Chapter 6. The Radial breathing mode in CdSe nanorods

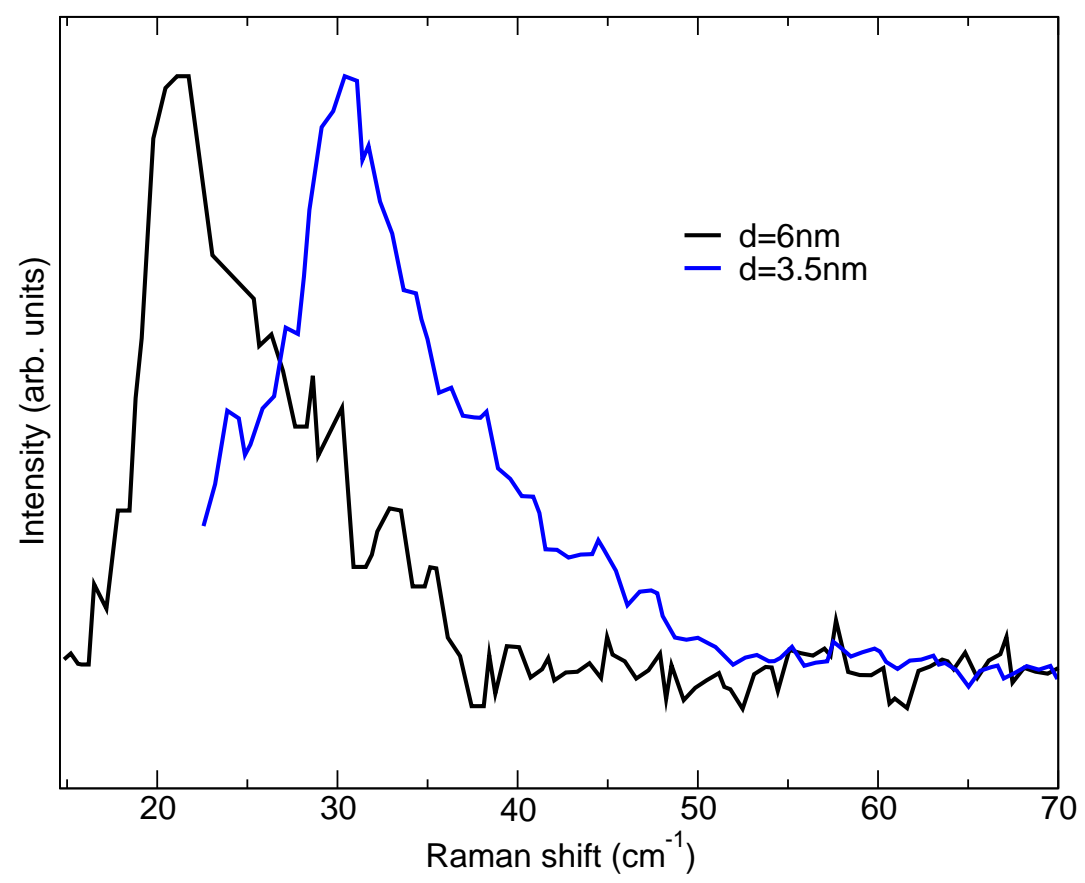

Figure 6.5: Raman spectra from CdSe at liquid helium temperature. The laser-related background is subtracted.

nanorod samples with different diameters are displayed in Figure 6.5.

The mode shows a dependence on the nanorod diameter. The frequency increases for a decreasing nanorod diameter. The measured RBM frequency versus diameter dependence can be described by a const./ $d$-function. The RBM frequencies are close to the expected frequencies from the linear elasticity and ab-initio models. The measured and ab-initio-calculated frequencies are summarized in Figure 6.6.

To estimate the nanorod diameter from a Raman measurement of the RBM, the constant factor for the $1 / d$-dependence is needed. It can be obtained by fitting const./ $d$ to all values, measured and calculated. The validity of the constant factor can be improved by weightening the individual data points according to their relative accuracy resulting from the experimental error, the fit correctness and the approximated accuracy of the calculations. The resulting dependence for the RBM frequency is $\omega_{\mathrm{RBM}}(d)=91.0 \mathrm{~cm}^{-1} \mathrm{~nm} / d$.

For small nanorods, this mode can be used to estimate the nanorod diameter from a Raman measurement. For nanorods with diameters above $6 \mathrm{~nm}$, the radial breathing modes is at very low frequencies. This makes a Raman measurement difficult. The mode can hardly be separated from the increasingly strong laser-related background. The radial breathing mode from nanorods of $8 \mathrm{~nm}$ diameter can not be 
6.4 Raman measurement of the radial breathing mode

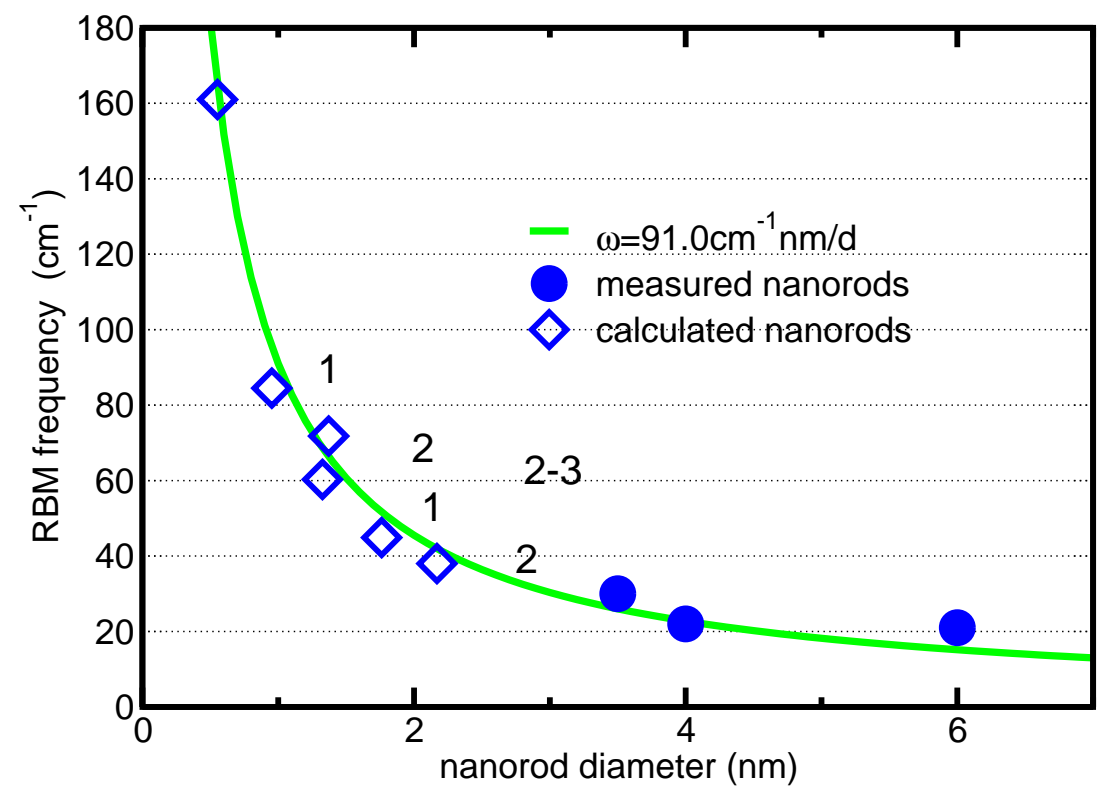

Figure 6.6: Measured and calculated radial breathing mode frequencies versus nanorod diameter.

detected with the used Raman set-up. Low-wavenumber Raman measurements are limited by the bandwidth of the filter system applied to suppress the Rayleigh line. Metal vapors with very narrow absorption lines can be applied as filters. Instead of using monochromators or dielectric filters to suppress the Rayleigh scattered light, the wavelength of a narrow-band tunable laser can be tuned to the narrow absorption lines of metal vapors, which then work as filters[93]. The inaccessible area around the Rayleigh line can then be reduced to less than $1 \mathrm{~cm}^{-1}$. The width of the absorption band can be changed by the conditions of the vapor cell. This is however not appropriate for the size determination of CdSe nanorods, as it would require an additional set-up just for the low-frequency measurements of the larger nanorods, while TEM becomes more powerful with increasing nanorod size. For small diameters the RBM can be useful to determine the nanorod diameter simultaneously with other Raman experiments. It can furthermore be used to estimate diameter distributions in nanorod ensembles. In spherical particles of similar composition, the line-shape of the spherical equivalent to the RBM can be used to estimate the size distribution of the investigated particles[94]. There, the agreement between the distributions from the Raman spectra and TEM measurements is quite good.

In this chapter a diameter dependent radial breathing mode was presented and its existence in nanorods was demonstrated by Raman spectroscopy. The diameter dependence of the RBM frequency can be used to determine the nanorod diameter by 
Chapter 6. The Radial breathing mode in CdSe nanorods

a Raman measurement. 


\section{Chapter 7}

\section{Core-shell nanorods}

As presented in Chapter 2, the nanorods can be coated with an epitaxial shell. The shell has an influence on the optical properties of the nanorod core, e.g. the photostability, exciton lifetime and other photoluminscent properties[11, 20]. The shell is crucial for many application, as it enables a further functionalization of the surface and for example water solubility or the embedding of the nanorods in polymers. The lattice mismatch between the nanorod core material, CdSe, and the shell material, ZnS, introduces strain into the lattice of the CdSe nanorod and the $\mathrm{ZnS}$ shell is strained itself. This means that not only the boundary of the nanorod is changed by the addition of the shell, but also the nanorod core. For example, it has been shown that the band alignments in the core-shell heterostructures are highly affected by strain. Depending on the thickness of the shell and core, heterostructures of type I and type II can be created[95]. As shown in Chapter 3 the bonds in the lateral direction of the CdSe core are homogeneously compressed. In this chapter, Raman spectroscopy is applied in order to estimate the amount of strain in the physical nanorods. The influence of the shell on the properties and effects that were presented in the previous chapters is discussed.

\subsection{Influence of the shell on the Raman spectra}

To investigate the influence of the ZnS shell, Raman spectra of bare nanorods and core-shell nanorods are compared. The Raman spectrum of CdSe nanorods is significantly changed when an epitaxial ZnS shell is added to the CdSe nanorods. The Raman spectrum of CdSe nanorods with $8 \mathrm{~nm}$ diameter and $80 \mathrm{~nm}$ length, capped with a $2-3$ monolayer $\mathrm{ZnS}$ shell is displayed in Figure 7.1. Included in the figure is the fit to a spectrum of the corresponding bare nanorods. The band at $520 \mathrm{~cm}^{-1}$ 
Chapter 7. Core-shell nanorods

in the spectra is not related to the nanorods. It stems from the Si-wafer on which the samples are deposited.

When comparing the fits to the two spectra one finds the following changes that happen with the addition of the shell: The LO related band is shifted to higher wavenumbers, the SO frequency is highly influenced by the shell and a new Raman band appears around $280 \mathrm{~cm}^{-1}$. The $\mathrm{ZnS} \mathrm{LO}$ is not observed in the Raman spectra, propably due to the small shell thickness of only a few monolayers. In investigations of spherical CdSe nanocrystals with ZnS shells, the ZnS LO can only be well separated from the CdSe related features for shell thicknesses above 3 monolayers[68]. A further increase in shell thickness then leads to an increase of the $\mathrm{ZnS}$ LO bands integral intensity, which is roughly proportional to the $\mathrm{ZnS}$ volume. This should also be valid for nanorods.

The shift of the LO frequency due to a decreased phonon confinement because of its (partial) propagation into the shell is not likely because the LO phonon dispersion curves of bulk CdSe and $\mathrm{ZnS}$ are not overlapping, and the $\mathrm{ZnS}$ shell is thus not expected to support the CdSe-like LO vibrations[96]. The shift of the LO frequency can explained by compressive strain. The changed bonding lengths due to strain in the CdSe core result in changed phonon frequencies. The estimation of the frequency shift allows to estimate the amount of compressive strain in the nanorod core. This will be used later.

Following the discussion in Chapter 4, the surrounding of the nanorod is crucial for the $\mathrm{SO}$ frequency. The $\mathrm{ZnS}$ shell has a different dielectric constant than air and is thus responsible for the large changes in the observed $\mathrm{SO}$ frequencies.

The Raman band at $280 \mathrm{~cm}^{-1}$ can have different origins. It could be attributed to the $\mathrm{ZnS}$ transverse optical phonon[28]. Otherwise, the $\mathrm{ZnS}$ shell deposition could be accompanied by core-shell material interdiffusion and the formation of an intermixed interface layer, manifesting itself in the Raman spectra as a peak at $280-290 \mathrm{~cm}^{-1}[96]$. Dzhagan et al. investigated the influence of different shell parameters on phonons in spherical CdSe core-shell nanoparticles. There, the peak at $280 \mathrm{~cm}^{-1}$ was observed for both $\mathrm{CdS}$ and $\mathrm{ZnS}$ shells. The authors of reference [96] attribute it thus to the Cd-S vibration in an interface layer.

\subsection{Strain in core-shell nanorods}

The quasi-uniaxial compression of the lateral bonds in the CdSe core as shown in Chapter 3 can be experimentally investigated by Raman spectroscopy. The effect of an uniaxial strain on the frequency of the crystal vibrations can be discussed within the theory established by Eduard Grüneisen. In 1912 and 1918 Grüneisen published articles on the thermal expansion of crystalline solids[97, 98]. Even without the 


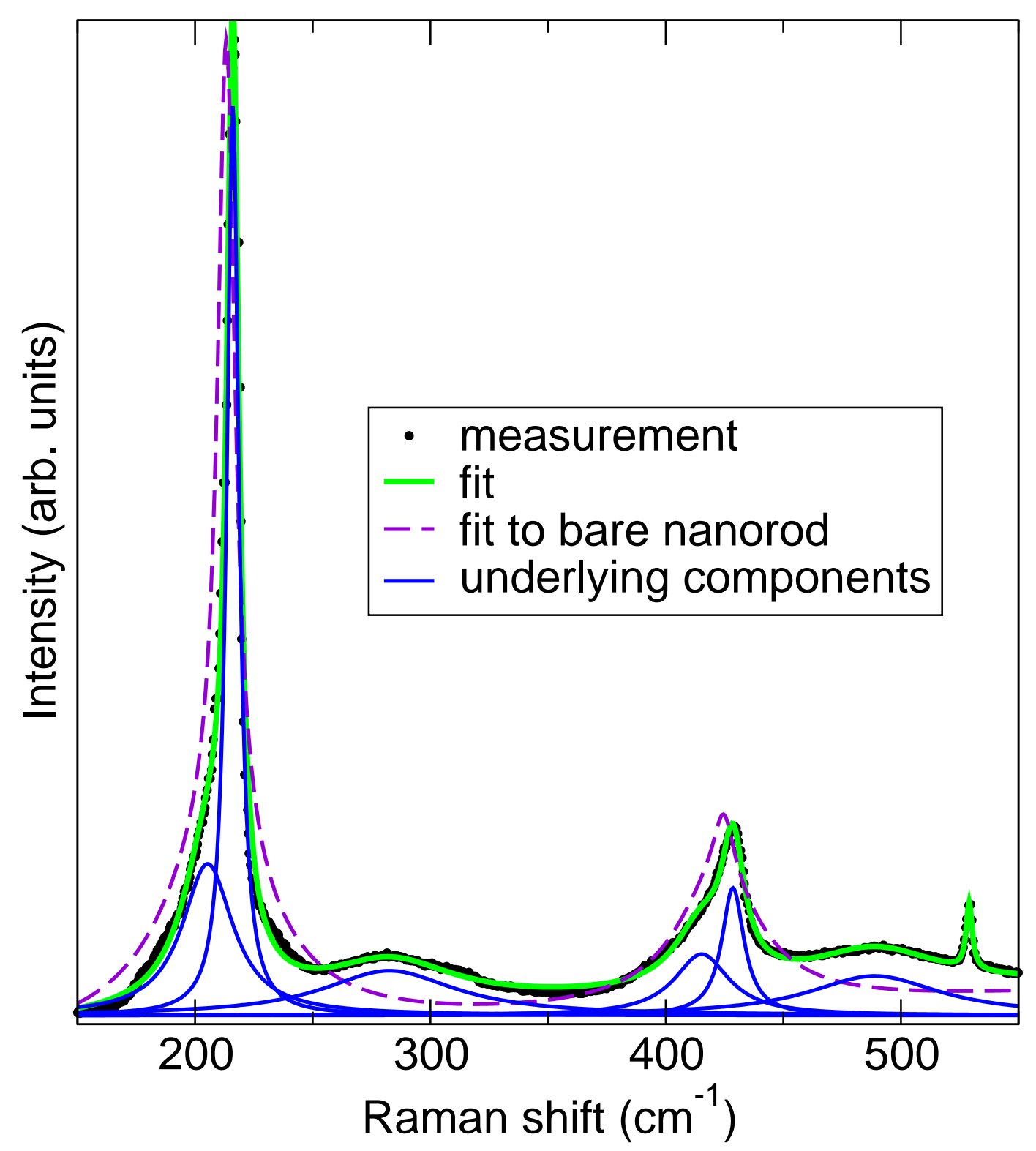

Figure 7.1: Raman spectrum from CdSe nanorods of $8 \mathrm{~nm}$ diameter and $80 \mathrm{~nm}$ length with a $2-3$ monolayer ZnS shell 
Chapter 7. Core-shell nanorods

knowledge of quantum mechanics and the nature of the forces between atoms, his discussion is still valid. Grüneisen assumes the thermal vibrations of atoms to be undamped harmonic oscillations. He then evaluates the influence of a single moved atom on its neighboring atoms by considering attracting and repelling forces (phenomenological at that time), which scale with $-\frac{a}{r^{x}}+\frac{b}{r^{y}}$. Next he investigates the change of the atomic vibrations with increasing pressure and temperature. This results in $\gamma=-\frac{\partial \ln \omega}{\partial \ln V}=-\frac{V(\partial V / \partial T)_{p}}{C_{v}(\partial V / \partial p)_{T}} . \quad C_{v}$ is the energy of a single oscillator. $\gamma$ is a material dependent constant and was later named Grüneisen parameter. $\omega$ is the frequency of the crystal vibration and $V$ is the crystal volume. For constant temperatures, uniaxial strain and within the current understanding of phonons in crystals, the Grüneisen parameter can be used to relate changes of the phonon frequencies to strain. The Grüneisen parameter for longitudinal optical phonons in CdSe was determined by experiments and is approximately 1.1[99]. The relative shift of the LO frequency is related to strain by [100]

$$
\frac{\Delta \omega}{\omega}=\left(1+3 \frac{\Delta a}{a}\right)^{-\gamma}-1,
$$

where $a$ is the lateral lattice parameter. Measuring the difference of the LO frequency relative to unstrained bulk material then allows a direct calculation of the strain within the crystal lattice.

A Raman spectrum of thin, bare CdSe nanorod with $2.5 \mathrm{~nm}$ diameter and $15 \mathrm{~nm}$ length is displayed in Figure 7.2. Due to confinement effects the luminescence of the nanorods is at lower wavelengths than for bulk material. This is why a slight luminescent background is present in the Raman spectra. It can be fitted with a quadratic polynominal. As presented in Chapter 4, the fundamental Raman band has an asymmetric shape. The measured longitudinal optical phonon frequency deviates from the bulk CdSe LO frequency. Two effects contribute to the frequency shift: the previously discussed phonon confinement and compressive strain caused by the surface reconstruction.

To estimate the amount of strain caused by the surface reconstruction, the deviation of the measured longitudinal optical phonon frequency from the frequency of an unstrained nanorod of the same diameter has to be evaluated. Due to phonon confinement, the frequency of unstrained CdSe nanorods differs from the bulk CdSe LO frequency and is, according to the phonon confinement model, at $\omega=210 \mathrm{~cm}^{-1}$ for nanorods of $2.5 \mathrm{~nm}$ diameter. The measured LO frequency of the bare nanorods is $\omega=211.3 \mathrm{~cm}^{-1}$, which corresponds to a surface reconstruction related strain of $\Delta a / a=-0.2 \%$. This is within the expectations from the $a b$-initio calculation results, which result in $\Delta a / a=-0.64 \%$ for much thinner nanorods with a diameter of $1.4 \mathrm{~nm}$. As in the calculations, the influence of the surface reconstruction vanishes 
7.2 Strain in core-shell nanorods

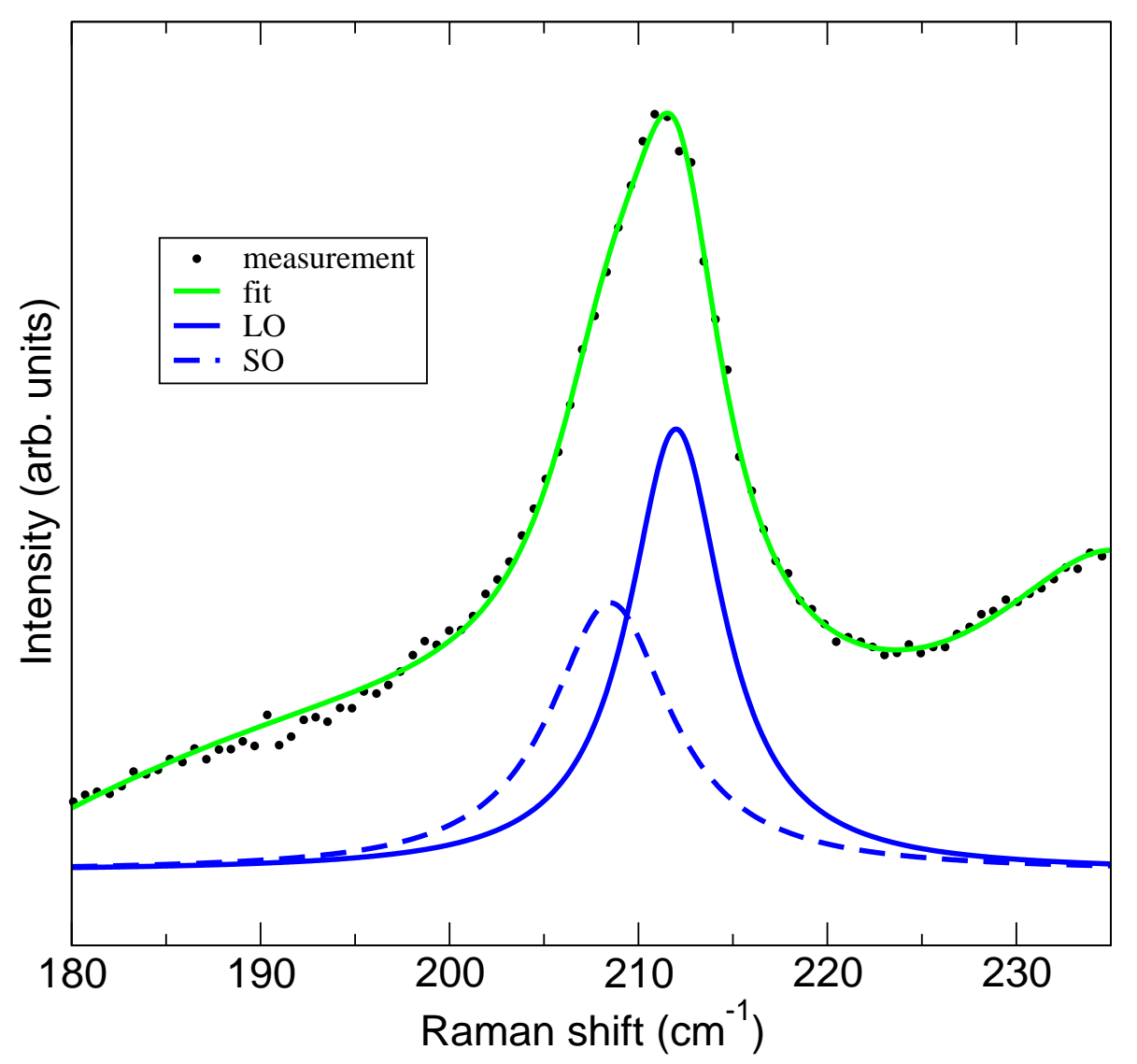

Figure 7.2: Raman spectrum of bare CdSe nanorods with $2.5 \mathrm{~nm}$ diameter.

for increasing diameters. The relative difference of the measured LO frequency from the expected LO frequency from the confinement model is below the experimental error of $\approx 0.4 \mathrm{~cm}^{-1}$ for nanorods with a diameter of $4 \mathrm{~nm}$ and $8 \mathrm{~nm}$. The influence of the surface reconstruction can be neglected for nanorods with diameters clearly above $2.5 \mathrm{~nm}$.

To investigate the strain caused by an added $\mathrm{ZnS}$ shell, spectra from bare CdSe nanorods and corresponding spectra from core-shell nanorods are compared. A Raman spectrum from nanorods of $2.5 \mathrm{~nm}$ diameter covered with one monolayer $\mathrm{ZnS}$ shell is displayed in Figure 7.3. As mentioned in Chapter 2, the luminescence efficiency of the core-shell nanorods is larger than that of the bare nanorods. This leads to an increased luminescent background. The LO frequency of the nanorod covered with a single monolayer of $\mathrm{ZnS}$ is at $\omega=212.4 \mathrm{~cm}^{-1}$. This corresponds to a compressive strain of $\Delta a / a=-0.5 \%$. A second $\mathrm{ZnS}$ monolayer increases this effect. The LO frequency is further shifted to $212.0 \mathrm{~cm}^{-1}$. This is due to a total strain 
Chapter 7. Core-shell nanorods

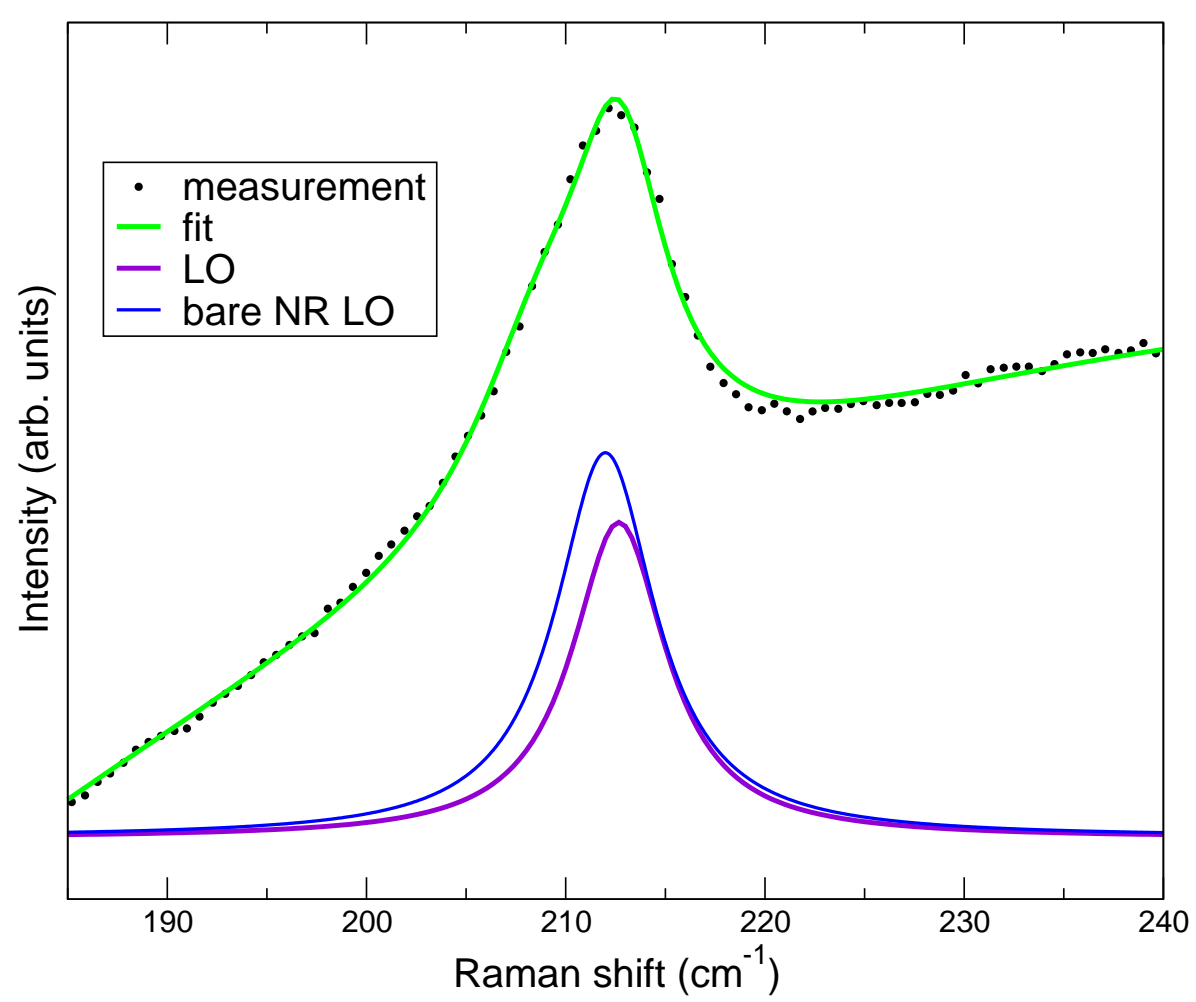

Figure 7.3: Raman spectrum of CdSe nanorods with $2.5 \mathrm{~nm}$ diameter with a one monolayer $\mathrm{ZnS}$ shell. The LO band from the corresponding bare nanorod is included for comparison.

of $\Delta a / a=-0.7 \%$. These values are within the expectations from the ab-initio calculations in Chapter 3.

The influence of the shell is expected to decrease for increasing nanorod core diameter. To examine the size-dependence of the shells' influence, measurements on core/core-shell pairs of thicker nanorods are performed. Raman spectra of CdSe nanorods with $4 \mathrm{~nm}$ diameter and $25 \mathrm{~nm}$ length, with and without a two-monolayer ZnS shell, are displayed in Figure 7.4.

Due to a changed confinement, the luminescence wavelength of the nanorods differs from the smaller $2.5 \mathrm{~nm}$ diameter nanorods. A luminescent background is not present in the spectra. The background can be fitted with a constant. The LO frequency of the $4 \mathrm{~nm}$ diameter nanorods is shifted from $\omega=210.0 \mathrm{~cm}^{-1}$ to $\omega=210.9 \mathrm{~cm}^{-1}$ between the bare and the core-shell nanorods, which results from a compressive strain of $\Delta a / a=-0.2 \%$ in the core-shell nanorods. For the third, largest nanorod sample pair, nanorods with $8 \mathrm{~nm}$ diameter, the LO frequency shifts from $\omega=215.2 \mathrm{~cm}^{-1}$ to $\omega=217.1 \mathrm{~cm}^{-1}$. Compressive strain due to the shell growth is almost not present in 


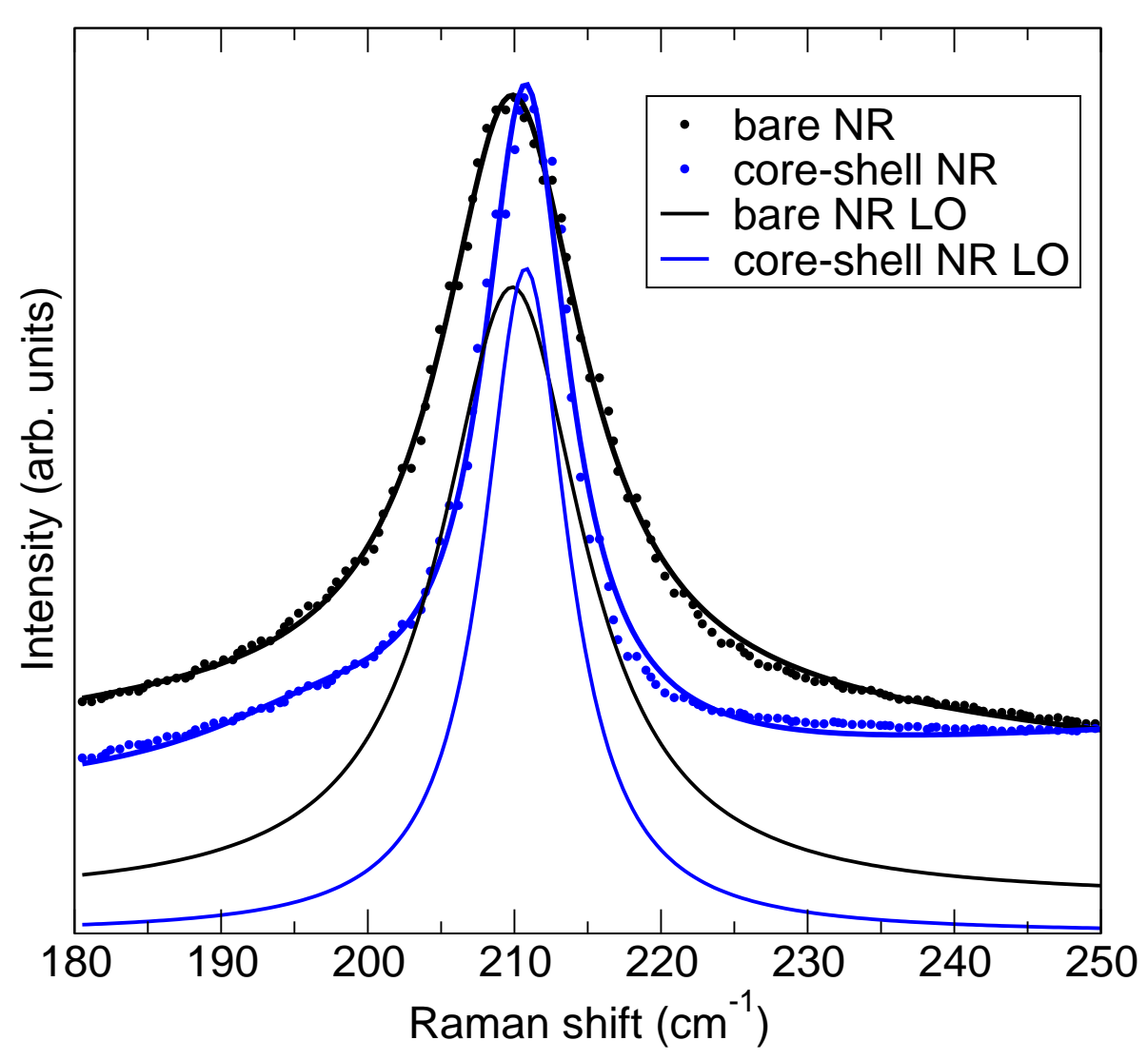

Figure 7.4: Raman spectra of bare CdSe nanorods with $4 \mathrm{~nm}$ diameter and of CdSe-ZnS core-shell nanorods with the same diameter.

such structures. It calculates to $\Delta a / a=-0.1 \%$. The strain values for the different nanorod geometries are summarized in Table 7.1.

The geometries with a length of $\infty$ are the calculation results. The qualitative agreement between experiment and theory shows that strain in core-shell nanorods can be treated as uniaxial compression and estimated with the CdSe bulk Grüneisen parameter. The strain values are also in qualitative agreement with experiments on $\mathrm{CdSe} / \mathrm{ZnSe}$ nanocrystals. Lee et al. investigated the lattice distortion and luminescence of spherical CdSe-ZnSe core-shell nanocrystals with approx. $3 \mathrm{~nm}$ diameter by X-ray diffraction and photoluminescence spectroscopy[39]. The authors find a decrease of $1.234 \%$ and $1.511 \%$ for the parameters $a$ and $c$, respectively. The nanocrystals they investigate are spherical, which explains the similarity in the decrease of $a$ and $c$, in contrast to the cylindrically shaped nanorods, where the change in $c$ can be neglected.

The strain in the nanorods increases for increasing shell-sizes. However, the shells' 
Chapter 7. Core-shell nanorods

Table 7.1: Relative strain values of the investigated CdSe nanorod samples and calculated values.

\begin{tabular}{c|c} 
sample type & $\Delta a / a$ \\
\hline $1.4 \mathrm{~nm} \times \infty$ & $-0.64 \%$ \\
$2.2 \mathrm{~nm} \times \infty$ & $-0.31 \%$ \\
$1.4 \mathrm{~nm} \times \infty$ w. $1 \mathrm{ML} \mathrm{ZnS}$ & $-1.75 \%$ \\
$1.4 \mathrm{~nm} \times \infty$ w. $2 \mathrm{ML} \mathrm{ZnS}$ & $-2.29 \%$ \\
$2.5 \mathrm{~nm} \times 15 \mathrm{~nm}$ & $0.2 \%$ \\
$2.5 \mathrm{~nm} \times 15 \mathrm{~nm}$ w. $1 \mathrm{ML} \mathrm{ZnS}$ & $-0.4 \%$ \\
$2.5 \mathrm{~nm} \times 15 \mathrm{~nm}$ w. $2 \mathrm{ML} \mathrm{ZnS}$ & $-0.7 \%$ \\
$4 \mathrm{~nm} \times 25 \mathrm{~nm}$ & - \\
$4 \mathrm{~nm} \times 25 \mathrm{~nm} .2 \mathrm{ML} \mathrm{ZnS}$ & $-0.2 \%$ \\
$8 \mathrm{~nm} \times 80 \mathrm{~nm}$ & - \\
$8 \mathrm{~nm} \times 80 \mathrm{~nm} .2 \mathrm{ML} \mathrm{ZnS}$ & $-0.1 \%$
\end{tabular}

crystal structure also improves with increasing shell dimensions. As shown in Chapter 2, the improved surface passivation and reduced leakage of the exciton wave function leads to an improved quantum yield until, at a certain shell-size, the straininduced stacking faults lead to a reduction of the quantum yield. Concerning the shell thickness for optical applications, there is a trade-off between strain and straininduced defects and the improved exciton localization and surface passivation.

\subsection{Surface optical phonons in core-shell nanorods}

As mentioned at the beginning of this chapter, the surface optical phonons are strongly affected by the changed nanorod surrounding due to the shell growth. Using the dielectric constant of $\mathrm{ZnS}, \epsilon_{D}=9.6$ [28], for the host material for equation 4.12 results in changed surface optical phonon frequencies. The direction of the SO frequency shift is the same for experiment and theory. However, these frequencies are shifted much stronger in theory than the experimentally observed ones. The calculation of the SO frequency involves the dielectric constant of the surrounding medium, which is a macroscopic parameter. This is obviously not suitable for 2-3 monolayers of ZnS. To reflect the nature of the shell, an effective dielectric constant can be used. When the dielectric constant of the host material is used as fit parameter $\epsilon^{\prime} D=2.9$ is obtained as effective dielectric constant. This is much smaller than the bulk dielectric constant of $\mathrm{ZnS}$ and could indicate a partial coverage of the nanorod, a not purely epitaxial shell, or reflect the thin nature of the shell and a resulting "mixed" dielectric constant of air and ZnS. However, this only explains the 
7.4 Shell-influence on the exciton-phonon coupling

trends of the observations. The model for surface optical phonons does not allow to deduce shell parameters from this effective dielectric constant.

\subsection{Shell-influence on the exciton-phonon coupling}

The ratio of integrated intensities of the LO-overtone to LO-fundamental Raman band is significantly reduced for the nanorods coated with a $\mathrm{ZnS}$ shell. This means that the Huang-Rhys parameter decreases for the core-shell nanorods. The HuangRhys parameter is reduced by $18 \%$ for the nanorods with diameters of $7 \mathrm{~nm}$ and above and reduced by more than $40 \%$ for CdSe nanorods with diameters below $5 \mathrm{~nm}$ when a $\mathrm{ZnS}$ shell is added.

Due to the compressive strain, the diameter of the CdSe core-shell nanorods is reduced compared to the corresponding bare nanorods. According the models and experiments presented in Chapter 5, the Huang-Rhys parameter increases for a decreasing diameter, opposite to the observations. Even if the shell is accounted for by an increase in the total diameter, which would then result in a reduced HuangRhys parameter, the observed changes are much larger than one would expect from the diameter dependence. From extrapolating the trend of the diameter dependent measurements, the increase in diameter due to the additional shell would lead to changes less than $2 \%$. Furthermore, the shell has a much greater impact on the small nanorods than on the larger structures, which is also not reflected by the diameter dependence. The Huang-Rhys parameter of the bare small nanorods is larger than that of the larger nanorods. For the coated nanorods, however, this is reversed. The Huang-Rhys parameter of small core-shell nanorods is smaller than that of the coated larger nanorods.

The difference in $S$ observed between nanorods with and without $\mathrm{ZnS}$ shell can be explained by a different confinement of the electron and hole wave function. An increase in the confining potential well as the result of the changed dielectric confinement due to the $\mathrm{ZnS}$ shell growth results in a reduced polarity of the exciton wave function and thus a reduction of the coupling. Another reason for the decreased coupling which might strengthen the effect of the confinement could be the reduced Fröhlich coupling in inferface regions with mixed fractions of $\mathrm{Cd}$ and $\mathrm{Zn}$. Zheng et al. did a theoretical investigation of exciton-LO-phonon interactions in zinc compounds and discovered a reduced electron-LO-phonon interaction in mixed $\mathrm{Zn}_{x} \mathrm{Cd}_{1-x}$ Se-compounds with rising $\mathrm{Zn}$ content[101]. This would be an explanation for the large deviation in the shift of the Huang-Rhys factor between small and large nanorods. The surface-to-volume ratio scales with $1 / r$, which means that small nanorods are subject much stronger to surface effects than the larger nanorods, and the amount of mixed $\mathrm{ZnCd}$-compounds comparative to the nanorod volume is 
increased. An estimation of the relative contributions of the two effects to the reduced coupling is not possible on the available experimental data. Dzhagan et al., however, included a discussion of the exciton-LO-phonon coupling in their investigation of the influence of different shell parameters on the phonons in spherical CdSe core-shell particles[96]. By comparing CdS and ZnS capped particles. The authors were able to monitor effects of the shell size and the dimensions of the intermixed region sperately and found the effect of the changed confinement of electron and hole to be more important for the changed coupling strength than the reduced Fröhlich coupling in the intermixed regions. Their discussion is general and this should also apply to the nanorods.

\subsection{The RBM in core-shell nanorods}

The radial breathing mode that was discussed in Chapter 6 is also present in the $a b$-initio calculated core-shell nanorods. A displacement pattern of the RBM in a core-shell nanorod with a two-monolayer ZnS shell is displayed in Figure 7.5.

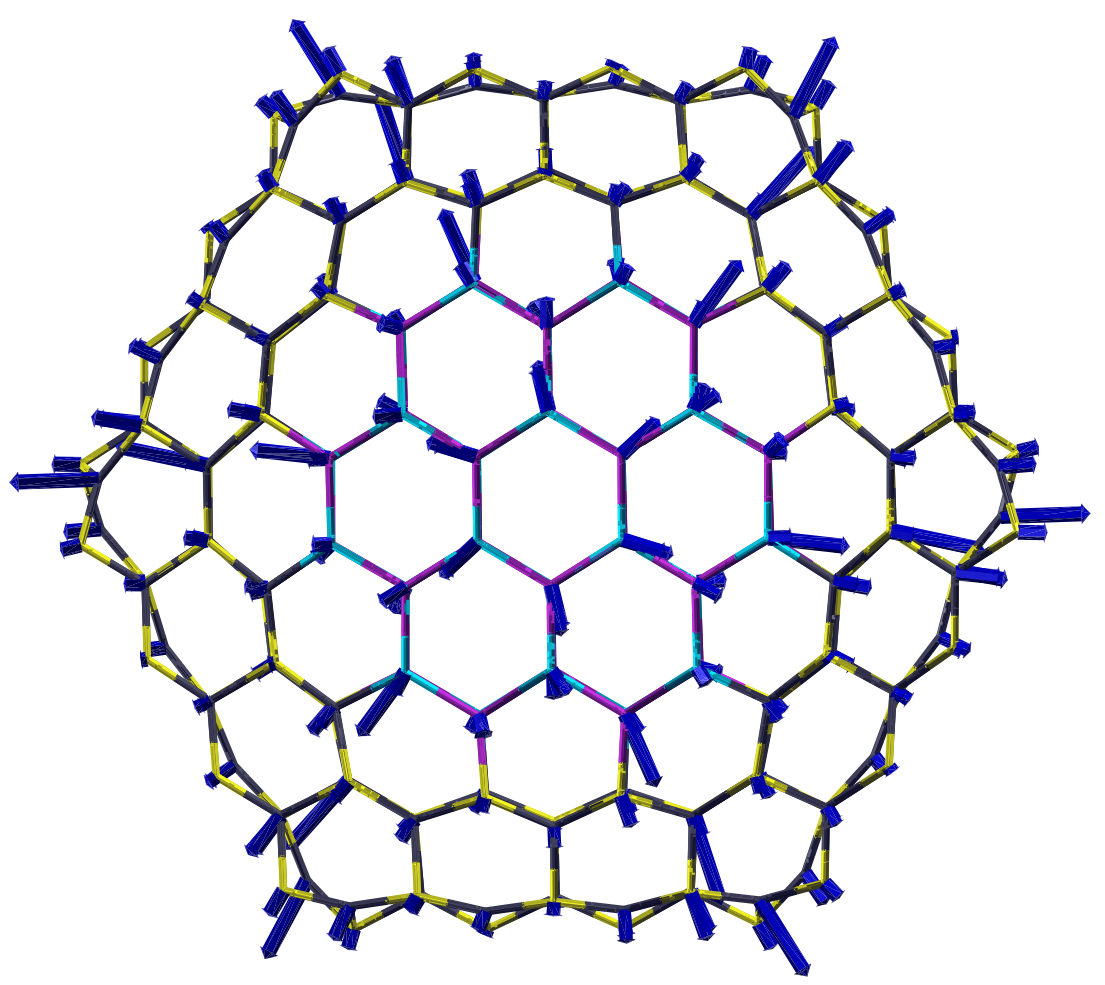

Figure 7.5: Displacement pattern of the radial breathing mode of a a $1.4 \mathrm{~nm}$ diameter core-shell CdSe-ZnS nanorod. Image courtesy of Marcel Mohr. 
The RBM extends to the shell. The RBM frequency is increased by $7-15 \mathrm{~cm}^{-1}$ compared to the bare counterpart. The thicker the shell, the larger the increase in frequency. This is due to the shortened bonding lengths in the CdSe core with the addition of the ZnS shell. The same effect can also be observed experimentally. The RBM frequency of a CdSe nanorod with a diameter of $2.8 \mathrm{~nm}$ and a length of $22 \mathrm{~nm}$ covered with a 2-3 monolayer ZnS shell is at $52 \mathrm{~cm}^{-1}$. This is $20 \mathrm{~cm}^{-1}$ higher than expected for a bare nanorod of this diameter. This increase of the RBM frequency is larger than for the calculated nanorods because the shell of the measured nanorods is thicker compared to the shells of the calculated nanorods. 



\section{Chapter 8}

\section{Summary and outlook}

In this work Raman spectroscopy was utilized to determine the formation of compressive strain in CdSe-ZnS core-shell nanorods, the exciton-phonon coupling strength and to investigate confinement effects.

The fundamental Raman band of CdSe nanorods consists of contributions from longitudinal optical phonons and surface optical phonons. The size-tunability of the nanorods was used to investigate the geometry-dependence of both contributions. The longitudinal optical phonons are confined to the small nanorod volume. This leads to a neutralization of the $\mathbf{q}=0$ rule and a shift of the longitudinal optical phonon frequency. The large aspect ratio of the nanorods results in a pure diameter dependence of this shift whereas the surface optical phonon frequency depends on the nanorods' aspect ratio. The sum of both effects leads to different positions and shapes of the fundamental Raman band with a change of the nanorod geometry. From this it follows that the size of the nanorods has always to be taken into account when discussing effects, where the absolute frequencies of the two contributions are relevant.

The coupling strength of the excitons to longitudinal optical phonons is accessible from the Raman spectra and was investigated experimentally. In all nanorods, it is about one order of magnitude smaller than the exciton-phonon coupling strength in bulk material. A decrease of the influence of the Coulomb interaction due to confinement effects is the origin of this. The size-tunability of the nanorods was utilized to investigate the size-dependence of the coupling-strength. The coupling strength rises for very small diameters because of an increased influence of higher frequency phonons with decreasing diameter.

The size tunability of the nanorods was also used to investigate a third size-dependent effect: A radial breathing mode was deduced from ab-initio calculations and experimentally characterized with Raman spectroscopy. The frequency of this mode is 
Chapter 8. Summary and outlook

diameter dependent and it thus allows the determination of the nanorod diameter by a Raman measurement alone.

The influence of an epitaxial coating around the nanorods was then investigated with Raman spectroscopy. Adding a ZnS shell around a CdSe nanorod strains the nanorod core because of the lattice mismatch between the core and shell material. The strain is mostly uniaxial on the lateral bonds and Raman spectroscopy yields strain values in agreement with ab-initio calculated values. The influence of the shell on the previously discussed effects in bare nanorods was investigated. The exciton-phonon coupling strength is smaller in core-shell nanorods than in bare nanorods due to a different confinement of the electron and the hole wavefunction and the radial breathing mode is also present in core-shell nanorods, but with an increased frequency due to the shortened bonds of the core. In conclusion, Raman spectroscopy is a versatile, non-destructive method to gain information on colloidal nanorods. With the results presented in this work Raman spectroscopy can now be used to estimate the exciton-phonon coupling strength, observe phonon confinement and determine the diameter of colloidal CdSe nanorods.

Recently, ordered structures of colloidal nanoparticles gained much attention[47, 102, 103]. The assembly of small nanoparticles into macroscopic structures is an important topic in chemistry and and materials science. The combination of nanoparticles of different materials into a nanoparticle superlattice can result in a large variety of materials (metamaterials) with controlled chemical composition. The possibility to control the nanoparticle size, shape and composition, as provided by the colloidal nanoparticle synthesis, allows the tuning of the electronic, optical and magnetic properties of these building blocks[103, 104]. Lattice-mismatch strains can control nanoscale pattern formation and semiconductor-metal superlattices can be created through partial cation exchange of colloidal nanocrystals. Strain induces then the spontaneous formation of periodic structures[102].

Raman spectroscopy could be used to investigate certain aspects in these emerging materials. The vibrations of the superlattice are expected to give insight into the quality of the order in the superlattice and the strength of the coupling between the particles. In mixed semiconductor-metal ensembles, surface effects as presented in Chapter 3 can be used to characterize contacts, contact distances, relative particle distributions etc.. The presence and nature of coherent acoustic phonons, nanoacoustic waves, could also be investigated with Raman spectroscopy. A low density of carriers, created with a laser pulse, in the superlattices is expected to lead to a screening of the piezoelectric field due to the spatial separation of the electrons and holes between the different particles. This space-charge screening field can cause the strain within the semiconductor particles to change as a function of time and lead to nanoacoustic waves. These waves could be usefull for several applications and are expected to be measurable in an adjusted Raman set-up[105].

Raman spectroscopy will be a useful addition to PL and TEM investigations of 
new colloidal nanoparticle heterostructures and help in the characterization and optimization of these structures regarding applications. 



\section{Bibliography}

[1] X. W. J. Zhuang, Q. Peng, and Y. Li, Nature 437, 121 (2005).

[2] X. Peng, L. Manna, W. Yang, J. Wickham, E. Scher, A. Kadavanich, and A. Alivisatos, Nature 404, 59 (2000).

[3] L. Li, J. Hu, W. Yang, and A. Alivisatos, Nano Lett. 1, 349 (2001).

[4] Z. Hens, D. Vanmaekelbergh, E. J. Stoffels, and H. van Kempen, Phys. Rev. Lett. 88, 236803 (2002).

[5] C. Murray, D. Norris, and M. Bawendi, J. Am. Chem. Soc. 115, 8706 (1993).

[6] Y. Sun and Y. Xia, Science 298, 2176 (2002).

[7] Y. Yin and A. Alivisatos, Nature 437, 664 (2005).

[8] V. Puntes, D. Zanchet, C. Erdonmez, and A. Alivisatos, J. Am. Chem. Soc. 124, 12874 (2002).

[9] H. Yu and W. Buhro, Adv. Mat. 15, 416 (2003).

[10] D. Talapin, J. Nelson, E. Shevchenko, S. Aloni, B. Sadtler, and A. Alivisatos, Nano Lett. 7, 2951 (2007).

[11] L. Manna, E. Scher, L. Li, and A. Alivisatos, J. Am. Chem. Soc. 124, 7145 (2002).

[12] Z. Peng and X. Peng, J. Am. Chem. Soc. 124, 3343 (2002).

[13] L. Manna, E. Scher, and A. Alivisatos, J. Am. Chem. Soc. 122, 12700 (2000).

[14] S. Tavenner-Kruger, Y. Park, M. Lonergan, U. Woggon, and H. Wang, Nano Lett. 6, 2154 (2006).

[15] A. Shabaev and A. Efros, Nano Lett. 4, 1821 (2004). 
[16] W. Huynh, J. Dittmer, and A. Alivisatos, Science 295, 2425 (2002).

[17] D. Milliron, S. Hughes, Y. Cui, L. Manna, J. Li, L. Wang, and A. Alivisatos, Nature 430, 190 (2004).

[18] L. Li, J. Walda, L. Manna, and A. Alivisatos, Nano Lett. 2, 557 (2002).

[19] J. Hu, L. Li, W. Yang, L. Manna, L. Wang, and A. Alivisatos, Science 292, 2060 (2001).

[20] T. Mokari and U. Banin, Chem. Mat. 15, 3955 (2003).

[21] M. Kazes, D. Lewis, Y. Ebenstein, T. Mokari, and U. Banin, Adv. Mat. 14, 317 (2002).

[22] D. Gerion, F. Pinaud, S. Williams, W. Parak, D. Zanchet, S. Weiss, and A. Alivisatos, J. Phys. Chem. B 105, 8861 (2001).

[23] B. Dubertret, P. Skourides, D. Norris, V. Noireaux, A. Brivanlou, and A. Libchaber, Science 298, 1759 (2002).

[24] X. Gao, Y. Cui, R. Levenson, L. Chung, and S. Nie, Nature Biotechnology 22, 969 (2004).

[25] U. Hotje, C. Rose, and M. Binnewies, Solid State Sci. 5, 1259 (2003).

[26] A. G. Lehmann, M. Bionducci, and F. Buffa, Phys. Rev. B 58, 5275 (1998).

[27] H. Stokes, J. Gunter, D. Hatch, J. Dong, H. Wang, and J. Lewis, Phys. Rev. B 76, 12102 (2007).

[28] Landolt-Börnstein: Numerical Data and Functional Relationships in Science and Technology. Group III Condensed Matter, Vol. 41 Semiconductors, IIVI and I-VII Compounds; Semimagnetic Compounds. Berlin, Heidelberg: Springer (1999).

[29] S. B. Zhang, S. Wei, and A. Zunger, J. Appl. Phys., 83, 3192 (1998).

[30] T. Ohtsuka, J. Kawamata, and Z. Z. T. Yao, Appl. Phys. Lett. 65, 466 (1994).

[31] X. Duan, Y. Huang, R. Agarwal, and C. Lieber, Nature 421, 241 (2003).

[32] S. Kumar and T. Nann, Small 2, 316 (2006).

[33] A. Puzder, A. Williamson, N. Zaitseva, G. Galli, L. Manna, A. Alivisatos, A. Shabaev, and A. Efros, Nano Lett. 4, 2361 (2004). 
[34] D. Talapin, I. Mekis, S. Go1tzinger, A. Kornowski, O. Benson, and H. Weller, J. Phys. Chem. B 108, 18826 (2004).

[35] R. Xie, U. Kolb, J. Li, T. Basche, and A. Mews, J. Am. Chem. Soc. 127, 7480 (2005).

[36] N. L. Thomas, E. Herz, O. Schöps, U. Woggon, and M. Artemyev, Phys. Rev. Lett. 94, 016803 (2005).

[37] U. Woggon, J. Appl. Phys. 191, 081727 (2007).

[38] G. Bryant and W. Jaskolski, J. Phys. Chem. B 109, 19650 (2005).

[39] Y.-J. Lee, T.-G. Kim, and Y.-M. Sung, Nanotechnology 17, 3539 (2006).

[40] B. Dabbousi, J. Rodriguez-Viejo, F. Mikulec, J. Heine, H. Mattoussi, R. Ober, K. Jensen, and M. Bawendi, J. Phys. Chem. B 101, 9463 (1997).

[41] M. Hines and P. Guyot-Sionnest, J. Phys. Chem. 100, 468 (1996).

[42] X. Peng, M. Schlamp, K. Kadavanich, and A. Alivisatos, J. Am. Chem. Soc. 119, 7019 (1997).

[43] M. Nirmal, B. Dabbousi, M. Bawendi, J. Macklin, J. Trautman, T. Harris, and L. Brus, Nature 383, 802 (1996).

[44] C. Kagan, C. Murray, and M. Bawendi, Phys. Rev. B 54, 8633 (1996).

[45] D. Norris, A. Efros, M. Rosen, and M. Bawendi, Phys. Rev. B 53, 16347 (1996).

[46] V. Kulakovskii, G. Bacher, R. Weigand, T. Kummell, A. Forchel, E. Borovitskaya, K. Leonardi, and D. Hommel, Phys. Rev. Lett. 82, 1780 (1999).

[47] A. Titov and P. Kral, Nano Lett. 8, 3605 (2008).

[48] Z. Chen and S. O’Brien, ACS Nano 2, 1219 (2008).

[49] F. Widulle, S. Kramp, N. M. Pyka, A. Göbel, T. Ruf, A. Debernardi, R. Lauck, and M. Cardona, Physica B 263, 448 (1999).

[50] N. Mingo, Phys. Rev. B 68, 113308 (2003).

[51] Peter Y. Yu, Manuel Cardona. Fundamentals of Semiconductors. Berlin, Heidelberg: Springer (2005). 
[52] Neil W. Ashcroft, N. David Mermin: LinkSolid State Physics. New York,: Holt, Rinehart and Winston (1981).

[53] W. Kohn, Rev. Mod. Phys. 71, 1253 (1999).

[54] J. Pople, Angew. Chem. Int. Ed. 38, 1894 (1999).

[55] W. Kohn and L. Sham, Phys. Rev. 140, 1133 (1965).

[56] J. Soler, E. Artacho, J. GaleR, A. García, J. Junquera1, P. Ordejón, and D. Sánchez-Portal, J. Phys.: Cond. Matter 14, 2745 (2002).

[57] M. Machón, Dissertation, Technische Universität Berlin (2006), http://opus.kobv.de/tuberlin/volltexte/2006/1440.

[58] M. Mohr and C. Thomsen, Nanotechnology 20, 115707 (2009).

[59] M. Mohr and C. Thomsen, Phys. stat. sol. (b) 245, 2111 (2008).

[60] X. Gonze, J. Beuken, R. Caracas, F. Detraux, M. Fuchs, G. Rignanese, L. Sindic, M. Verstraete, G. Zerah, F. Jollet, et al., Comp. Mat. Science 25, 478 (2002).

[61] J. Perdew and Y. Wang, Phys. Rev. B 45, 13244 (1992).

[62] B. Bonello and B. Fernandez, J. Phys. Chem. Sol. 54, 209 (1993).

[63] D. M. Aruguete, M. A. Marcus, L. S. Li, A. Williamson, S. Fakra, F. Gygi, G. A. Galli, and A. P. Alivisatos, J. Phys. Chem. C 111, 75 (2007).

[64] C. Raman and K. Krishnan, Nature 121, 501 (1928).

[65] C. Raman, Nature 121, 619 (1928).

[66] S. Nie and S. Emery, Science 275, 1102 (1997).

[67] R. Stöckle, Y. Suh, V. Deckert1, and R. Zenobi, Chem. Phys. Lett. 318, 131 (2000).

[68] A. Baranov, Y. Rakovich, J. Donegan, T. Perova, R. Moore, D. Talapin, A. Rogach, Y. Masumoto, and I. Nabiev, Phy. Rev. B 68, 165306 (2003).

[69] F. Comas, C. Trallero-Giner, N. Studart, and G. E. Marques, Phy. Rev. B 65, 073303 (2002).

[70] R. Gupta, Q. Xiong, G. Mahan, and P. Eklund, Nano Lett. 3, 1745 (2003). 
[71] H. Richter, Z. Wang, and L. Ley, Solid State Commun. 39, 625 (1981).

[72] I. Campbell and P. Fauchet, Solid State Commun. 58, 739 (1986).

[73] J. Serrano, A. H. Romero, F. J. Manjon, R. Lauck, M. Cardona, and A. Rubio, Phys. Rev. B 69, 094306 (2004).

[74] M. Mohr, J. Maultzsch, E. Dobardžić, S. Reich, I. Milošević, M. Damnjanović, A. Bosak, M. Krisch, and C. Thomsen, Phys. Rev. B 76, 035439 (2007).

[75] F. Comas, C. Trallero-Giner, N. Studart, and G. E. Marques, J. Phys.: Cond. Matter 14, 6469 (2002).

[76] R. Ruppin and R. Englman, Rep. Prog. Phys. 33, 149 (1970).

[77] A. Sashchiuk, L. Amirav, M. Bashouti, M. Krueger, U. Sivan, and E. Lifshitz, Nano Lett. 4, 159 (2004).

[78] H. Fröhlich, Advances in Physics 3, 325 (1954).

[79] K. Huang and A. Rhys, Proc. R. Soc. London Ser. A 204, 406 (1950).

[80] D. Tannor and E. Heller, J. Chem. Phys. 77, 202 (1982).

[81] A. Albrecht, J. Chem. Phys. 34, 1476 (1961).

[82] C. Duke and G. Mahan, Phys. Rev. 139, 1965 (1965).

[83] R. Merlin, G. untherodt, R. Humphreys, M. Cardona, S. Suryanarayanan, and F. Holtzberg, Phys. Rev. B 17, 4951 (1978).

[84] T. Krauss and F. Wise, Phys. Rev. B 55, 9860 (1997).

[85] S. Nomura and T. Kobayashi, Phys. Rev. B 45, 1305 (1992).

[86] S. Smitt-Rink, D. Miller, and D. Chemia, Phys. Rev. B 35, 8113 (1986).

[87] G. Scamarcio, V. Spagnolo, G. Ventruti, and M. Lugará, Phys. Rev. B 53, 489 (1996).

[88] J. Maultzsch, H. Telg, S. Reich, and C. Thomsen, Phys. Rev. B 72, 205438 (2005).

[89] M. H. Comas, X. Wang, G. Hartland, P. Mulvaney, J. Juste, and J. Sader, J. Am. Chem. Soc. 125, 14925 (2003). 
[90] Love, A.E.J. A treatise on the Mathematical Theory of Elasticity. New York: Dover Publications (1944).

[91] T. Thonhauser and G. Mahan, Phys. Rev. B 71, 081307 (2005).

[92] C. Cline, H. Dunegan, and G. Henderson, J. Appl. Phys. 38, 1944 (1967).

[93] A. Nohe, G. Lermann, H. Schwoerera, W. Kiefer, J. Sawatzki, and G. Surawicz, J. Mol. Struc. 410, 65 (1997).

[94] M. Ivanda, K. Furić, S. Musić, M. Ristic, M. Gotić, A. Tonejc, I. Djerdj, M. Mattarelli, M. Montagna, F. Rossi, et al., J. Raman Spectrosc. 38, 647 (2007).

[95] J. Li and L. Wang, Appl. Phys. Lett. 84, 3648 (2004).

[96] V. Dzhagan, M. Valakh, E. Raevska, O. Stroyuk, S. Kuchmiy, and D. Zahn, Nanotechnology 20, 365704 (2009).

[97] E. Grüneisen, Ann. d. Physik 39, 257 (1912).

[98] E. Grüneisen, Ann. d. Physik 55, 371 (1918).

[99] A. P. Alivisatos, T. D. Harris, L. E. Brus, and A. Jayaraman, J. Chem. Phys. 89, 5979 (1988).

[100] G. Scamarcio, M. Lugara, and D. Manno, Phys. Rev. B 45, 13792 (1992).

[101] F. Zheng, M. Matsuura, and T. Taguchi, Phys. Rev. B 61, 9960 (2000).

[102] R. Robinson, B. Sadtler, D. Demchenko, C. Erdonmez, L. Wang, and A. Alivisatos, Science 317, 355 (2007).

[103] E. Shevchenko, D. Talapin, N. Kotov, S. O'Brien, and C. Murray, Nature 439, 55 (2006).

[104] E. Shevchenko, D. Talapin, C. Murray, and S. O'Brien, J. Am. Chem. Soc. 128, 3620 (2006).

[105] K. Lin, C. Lai, C. Pan, J. Chyi, J. Shi, S. Sun, C. Chang, and C. Sun, Nature Nanotechnology 2, 704 (2007). 


\section{Acknowledgments}

This work has been conducted with the help of others. Especially, I want to thank Christian Thomsen for the opportunity of working in his group and general support. Ulrike Woggon for the fruitfull cooperation and many advises.

Mikhail Artemyev for the sample growth and explanations concerning the synthesis. Marcel Mohr for the DFT calculations and many fruitful discussions.

Tore Nierman for the TEM measurements.

Patryk Kusch for his work on the temperature dependent properties.

María Machón for her support during the beginning of my work and proofreading. Robert Hauschild for proofreading.

All members of the Thomsen and Hoffmann groups for the good working atmosphere. 
